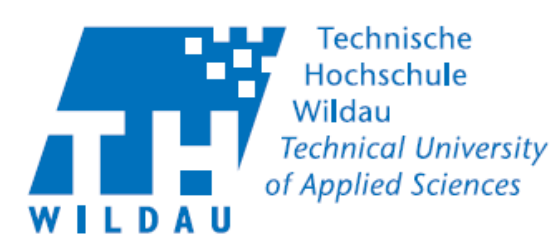

\title{
Masterarbeit
}

zur Erlangung des akademischen Grades

Master

Technische Hochschule Wildau

Fachbereich Wirtschaft, Informatik, Recht

Studiengang Bibliotheksinformatik (M.Sc.)

Thema (deutsch): Analyse der Katalogisierungssituation in Öffentlichen Bibliotheken Sachsen-Anhalts, sowie der Möglichkeiten von kostenfreien Datenübernahmen in nicht cloudbasierten Bibliotheksmanagementsystemen

Thema (englisch): Analysis of the cataloging situation in public libraries in Sachsen-Anhalt, and options of free of charge data migration in non cloud based library management systems

\footnotetext{
Autor/in: $\quad$ Andrea Kiefer

Matrikel-Nr.: $\quad 50030033$

Seminargruppe: $\quad$ BIM/15

Betreuer/in: Dipl.-Mathematiker Stefan Lohrum

Zweitgutachter/in: Dr. Frank Seeliger
}

Eingereicht am: $\quad$ 20.03.2019 


\section{Zusammenfassung/ Abstract}

Öffentliche Bibliotheken sind in Deutschland oft nicht an eine kooperative Katalogisierung in Bibliotheksverbünden angebunden. Durch die Einführung der Katalogisierungsregeln RDA, den Umstieg auf das Datenformat MARC21, aber auch durch die Freigabe der Metadaten einiger Bibliotheksverbünde und der DNB über eine CCO-Lizenz haben sich die grundlegenden Bedingungen der Katalogisierungsarbeit wesentlich verändert. Die Analyse einer durchgeführten Onlineumfrage beantwortet die Frage, ob die hauptamtlich geführten Öffentlichen Bibliotheken des Bundeslandes Sachsen-Anhalts diese Umstellung vollzogen haben. In Expertengesprächen wurden die technischen Möglichkeiten der eingesetzten Bibliotheksmanagementsysteme abgefragt und den Antworten der Bibliotheken gegenübergestellt.

Die Ergebnisse zeigen, dass bei einer großen Anzahl der Bibliotheken die Umstellung auf die neuen Anforderungen noch nicht vollzogen wurde und die neuen Möglichkeiten der Fremddatenübernahme häufig nicht genutzt werden oder nicht bekannt sind.

In einem Konzeptvorschlag ist eine mögliche Vorgehensweise für eine Verbesserung der derzeitigen Situation im Zusammenhang mit der Umgestaltung des ÖVK ausgearbeitet.

Dieses Konzept wird ergänzt durch Anleitungen und Leitfäden, in denen exemplarisch die möglichen Fremddatenübernahmen gezeigt werden.

Schlagwörter: Öffentliche Bibliothek; Katalogisierung; Fremddatenübernahme

Public libraries in Germany are often not integrated in cooperative cataloging of library affiliations. After introducing the new RDA cataloging rules, switching to the MARC21 format, and after publishing meta data of some library affiliations and of the DNB under a CCO license the prerequisites of cataloging has substantially changed. The analysis of a online survey answers the question if the full-time operated public libraries in Sachsen-Anhalt implemented this change. The technical capabilities of the library management systems in use have been explored in expert interviews and then compared to the answers of the libraries.

The results show that the change to the new requirements has not taken place yet in many libraries. In many cases the capability to import data is not used or even not known.

A conceptual proposal shows a possible approach to improve the current situation, in relation to the restructurization of the ÖVK.

The concept is supplemented by guidance and instructions showing by examples how to import foreign data.

Keywords: public library; cataloging; external data transfer 


\section{Inhaltsverzeichnis}

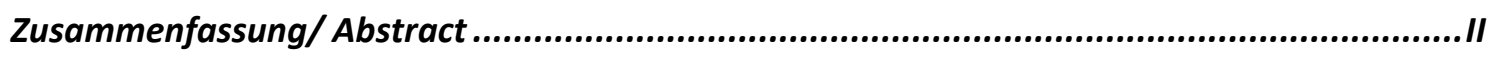

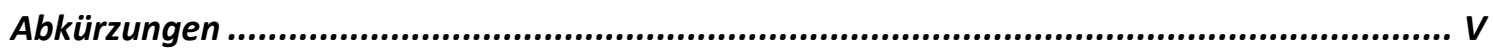

Tabellenverzeichnis .............................................................................................................. VII

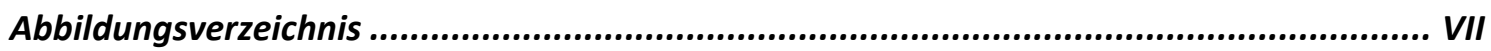

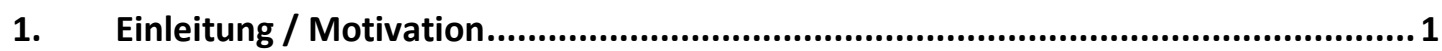

2. Öffentliche Bibliotheken in Sachsen-Anhalt ........................................................5

2.1. Begriffsbestimmung und Abgrenzung ……………................................................. 5

2.2. ÖVK - der Katalog der Öffentlichen Bibliotheken............................................................... 8

3. Aktuelle Situation der Öffentlichen Bibliotheken in Sachsen-Anhalt ....................11

3.1. Struktur und Gliederung der Institutionen .................................................................. 11

3.2. Statistischer 10-Jahres-Vergleich auf Grundlage der Deutschen Bibliotheksstatistik ......... 12

4. Einführung in die derzeitige Katalogisierungssituation ............................................16

4.1. Bibliothekarische Katalogisierung - Allgemeines ...................................................... 16

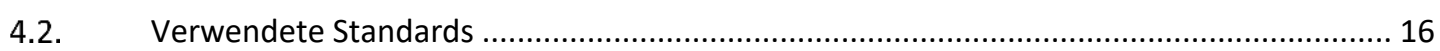

4.3. Unterscheidung der Katalogisierungsarbeit in Wissenschaftlichen und Öffentlichen

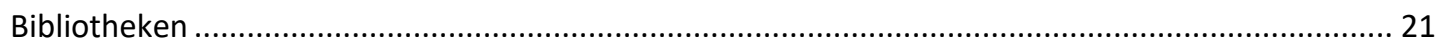

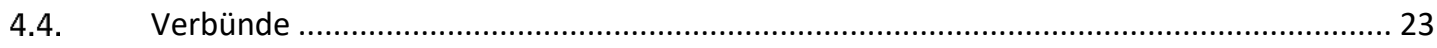

5. Umfrage zur Katalogisierungssituation in Öffentlichen Bibliotheken Sachsen-Anhalts 26

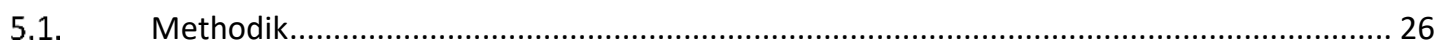

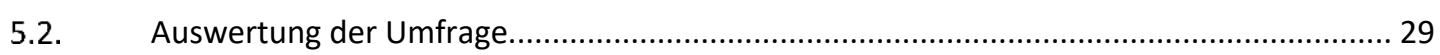

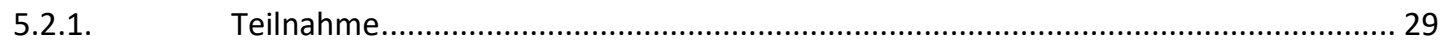

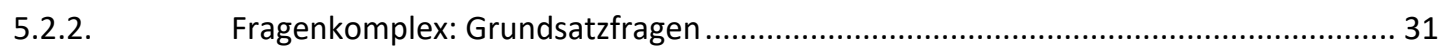

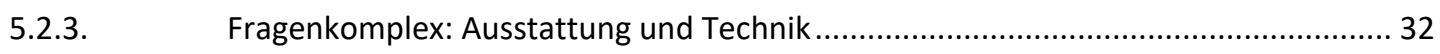

5.2.4. Fragenkomplex: Katalogisierung /Fremddatenübernahme ..................................... 35

5.3. Zusammenfassung und Überblick über die Umfrageergebnisse ..................................... 49

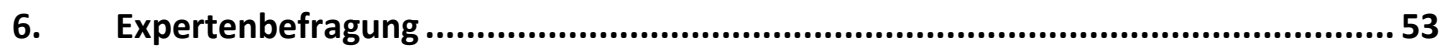

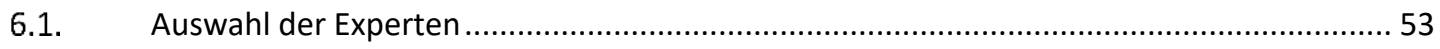

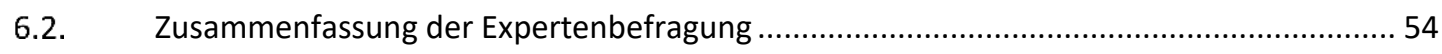

7. Möglichkeiten der kostenfreien Datenübernahme.........................................55

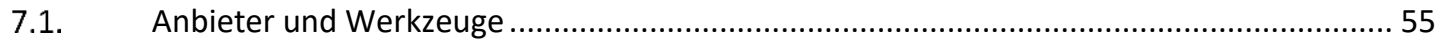

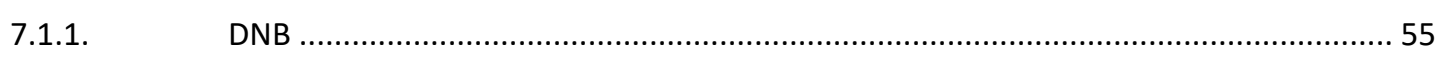

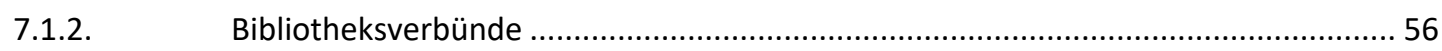

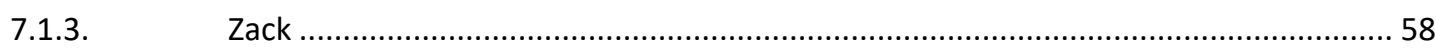


8. Fremddatenübernahme in den eingesetzten Bibliotheksmanagementsystemen ... 59

8.1. allegro-OEB - Büchereizentrale Niedersachsen.................................................... 59

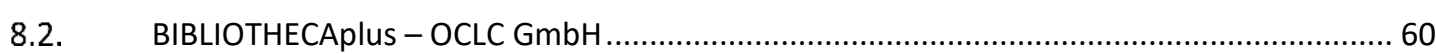

8.3. LIBRARY for Windows - Fleischmann Software Vertriebs $\mathrm{GmbH}$..................................60

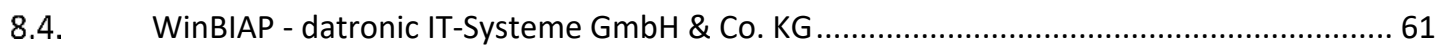

9. Cloudbasierte Angebote und mögliche Zukunft der Katalogisierung ....................63

10. Fazit............................................................................................. 64

11. Anleitungen / Leitfäden ...................................................................66 66

Literaturverzeichnis ................................................................................67

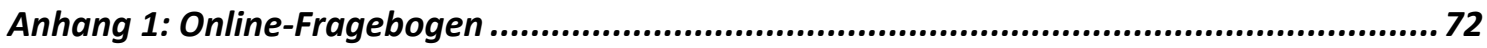

Anhang 2: Fragen an Anbieter der Bibliotheksmanagementsysteme.............................. 76

Anhang 3: Auswertungstabelle Expertenbefragung ..................................................... 77

Anhang 4: Anleitung Datenübernahme aus dem Datenshop der DNB ..............................81

Anhang 5: Anleitung Datenübernahme mit dem ZACK-Gateway ...................................83

Anhang 6: Konzeptvorschlag: Projekt Katalogisierung in Öffentlichen Bibliotheken Sachsen-

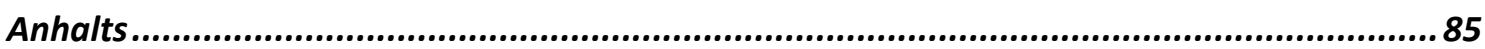

Anhang 7: Optionen zur Fremddatenübernahme in BIBLIOTHECAplus..........................87

Anhang 8: allegro-OEB.Dokumentation.Fremddatenübernahme .....................................95

Verzeichnis der auf der beiliegenden CD gespeicherten Dateien ................................... 102

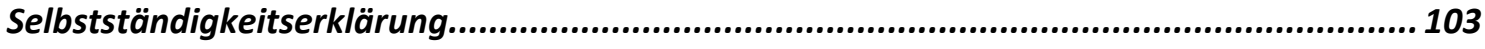




\section{Abkürzungen}

AGV

AIBM

Aleph

B3Kat

BIBFRAME

BOSS

BRISE

BSZ

BVB

CCO

DBS

DBV

DFG

DiViBib

DNB

ekz

FAMI

FRBR

FTP

GBV

GND

GVK

hbz

HeBIS

HTWK

i.d.R.

IAML

IFLA

ISBD
Arbeitsgemeinschaft der Verbundsysteme

Association Internationale des Bibliothèques, Archives et Centres de Documentation Musicaux (bis 2017 -jetzt: IAML)

Automated Library Expandable Program Hebrew University of Jerusalem

Gemeinsamer Verbundkatalog des Bibliotheksverbundes Bayern (BVB) und des Kooperativen Bibliotheksverbundes Berlin-Brandenburg (KOBV) Bibliographic Framework Transition Initiative BSZ One Stop Search Bibliotheksrecherche und Informationssystem Bibliotheksservice-Zentrum; Verbundzentrale des Südwestdeutschen Bibliotheksverbundes SWB Bibliotheksverbund Bayern Creative-Commons-Lizenz (bedingungslos frei)

Deutsche Bibliotheksstatistik

Deutscher Bibliotheksverband e.V.

Deutsche Forschungsgemeinschaft

divibib GmbH (Onleihe)

Deutsche Nationalbibliothek

ekz.bibliotheksservice $\mathrm{GmbH}$

Fachangestellte für Medien- und Informationsdienste

Functional Requirements for Bibliographic Records

File Transfer Protocol

Gemeinsamer Bibliotheksverbund; Verbundzentrale VZG

Gemeinsame Normdatei

Gemeinsamer Verbundkatalog (im GBV)

Hochschulbibliothekszentrum des Landes Nordrhein-Westfalen

Hessisches BibliotheksInformationsSystem

Hochschule für Technik, Wirtschaft und Kultur Leipzig

in der Regel

International Association of Music Libraries, Archives and Music Documentation Centres

International Federation of Library Associations and Institutions

International Standard Bibliographic Description 
ISIL

IT

$\mathrm{KAB}$

International Standard Identifier for Libraries and Related Organizations

KOBV

Informationstechnologie

K10plus

Klassifikation für Allgemeinbibliotheken

LSA

Kooperativer Bibliotheksverbund Berlin-Brandenburg

$M+M$

Gemeinsame Katalogisierungsdatenbank der Verbundzentralen BSZ und VZG

MAB / MAB2

Land Sachsen-Anhalt

MARC21

Match \& Merge (Verfahren zur Identifizierung von Datensatzdubletten)

MRM

Maschinelles Austauschformat für Bibliotheken (Deutschland)

NISO

Machine-Readable Cataloging (internationales Austauschformat)

OAl

Master Record Model

OCLC

National Information Standards Organisation

ÖB

Open Archives Initiative

Online Computer Library Center

ÖVK

Öffentliche Bibliothek

OPAC

Verbundkatalog Öffentlicher Bibliotheken (im GBV)

OPL

Online Public Access Catalogue

PICA

One Person Library

RAK

Project of Integrated Catalogue Automation

Regeln für die alphabetische Katalogisierung (durch RDA abgelöst)

RDA

Resource Description and Access (Internationales Regelwerk zur Katalogisierung)

SRU

Search/Retrieve via URL (Weiterentwicklung von Z39.50)

STA

Standardisierungsausschuss

SWB

Südwestverbund; Verbundzentrale BSZ

VLB

Verzeichnis lieferbarer Bücher

VZÄ

Vollzeitäquivalent

VZG

Verbundzentrale des GBV

WR

Wissenschaftsrat

WWW

World Wide Web

XML

Extensible Markup Language

Z39.50

Verbindungsorientiertes Netzwerkprotokoll für Suche in bibliographischen Informationssystemen

ZACK

Zack-Gateway 


\section{Tabellenverzeichnis}

Tab.: 1 Anzahl der hauptamtlich geführten Öffentlichen Bibliotheken in Sachsen-Anhalt nach Kreisen und Einwohnergrößen (Stand 2018) ....................................................... 12

Tab.: 2 Personal an Öffentlichen Bibliotheken in Sachsen-Anhalt - laut DBS in VZÄ .............. 15

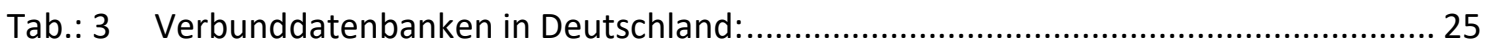

Tab.: 4 Verteilung der eingesetzten Bibliotheksmanagementsysteme der teilnehmenden Bibliotheken in Sachsen-Anhalt nach Einwohnergruppen $(n=40)$.

Tab.: 5 Verteilung der eingesetzten Katalogisierungsregeln zu den eingesetzten Bibliotheksmanagementsystemen

Tab.: 6 Anzahl und prozentuale Anteile an genutzten Fremddatenquellen aller Teilnehmerinnen $(n=49)$

Tab.: 7 Übersicht der Datenangebote der Verbünde.

\section{Abbildungsverzeichnis}

Abb.: 1 Darstellung der regionalen Suchmöglichkeit - Sicht für Sachsen-Anhalt -------------------9

Abb.: 2 Empfohlener Personalbedarf und IST-Zustand in Öffentlichen Bibliotheken SachsenAnhalts (zu betreuende Einwohner pro Vollzeitäquivalent - VZÄ) ------------------------ 14

Abb.: 3 Darstellung einer identischen Suche in GVK und ÖVK-------------------------------------- 22

Abb.: 4 Bibliotheksverbünde in Deutschland - nach Regionen----------------------------------- 23

Abb.: 5 Darstellung von Logik und Filter in der Fragestellung der Umfrage - eigene Darstellung -

Abb.: 6 Umfragebeteiligung der Öffentlichen Bibliotheken nach Einwohnerzahl der Gemeinden (Anzahl Bibliotheken; prozentualer Anteil)( $n=47)$

Abb.: 7 Prozentuale Verteilung der eingesetzten Bibliotheksmanagementsysteme der teilnehmenden Öffentlichen Bibliotheken in Sachsen-Anhalt $(n=40)$

Abb.: 8 Darstellung der Betreuung von Hardware und Server in den teilnehmenden Bibliotheken. Mehrfachantwort möglich ( $n=39)$

Abb.: 9 Verteilung der Bibliotheken mit Hosting des Bibliotheksmanagementsystems $(n=7) 34$

Abb.: 10 Gesamtpersonal in Sachsen-Anhalts Öffentlichen Bibliotheken nach Berufsgruppen, sowie anteiligem Einsatz in der Katalogisierung $(n=230,37)$ 
Abbildungsverzeichnis

Abb.: 11 Verteilung der Berufsgruppen in der Katalogisierung innerhalb der

Einwohnergruppen in VZÄ (n=94,42) -----

Abb.: 12 Prozentuale Verteilung der verwendeten Katalogisierungsregeln --------------------- 38

Abb.: 13 Prozentualer Anteil der teilnehmenden Bibliotheken mit und ohne Fremddatenübernahme $(n=39)$

Abb.: 14 Auflistung der Gründe für Nichtnutzung von Fremddaten - prozentualer Anteil und Anzahl Antworten( $n=13)$

Abb.: 15 Anwendung von Fremddatenübernahmen der teilnehmenden Bibliotheken in Sachsen-Anhalt nach Einwohnergröße ( $n=39)$

Abb.: 16 Anteile an manuell katalogisierten Mediengruppen aller Teilnehmenden der Umfrage $(n=55)$

Abb.: 17 Anteile manueller Katalogisierung bezogen auf Varianten der AV-Medien-

Abb.: 18 Beispieldarstellungen der anteiligen rein manuell erstellten Belletristik-

Titelaufnahmen aller Befragten im Vergleich zu Bibliotheken, in denen Fremddaten eingesetzt werden

Abb.: 19 Beispieldarstellungen der anteiligen rein manuell erstellten Titelaufnahmen der audiovisuellen Medien aller Befragten im Vergleich zu Bibliotheken, in denen Fremddaten eingesetzt werden

Abb.: 20 Prozentuale Darstellung der Wichtung der Katalogisierungskategorien bei der Fremddatenübernahme $(n=44)$

Abb.: 21 Verteilung der persönlichen Einschätzung der Relevanz der Katalogerweiterungen im OPAC $(n=60)$

Abb.: 22 Verteilung der persönlichen Einschätzung der Katalogisierungssituation in der eigenen Bibliothek( $n=59)$ 


\section{Einleitung / Motivation}

„Der Datenbestand des Bibliothekskatalogs [wird] heute nicht mehr von der eigenen Katalogisierung geprägt, sondern von der Übernahme von Fremddaten und dem damit verbundenen Metadatenmanagement. ${ }^{11}$ Klaus Gantert (2016)

Welche Bibliotheken mögen sich von dieser Einschätzung angesprochen fühlen? Öffentliche Bibliotheken sind vermutlich nicht gemeint. Dieser Verdacht drängt sich auch 2019 bei einem genaueren Blick auf die Katalogisierungspraxis in Öffentlichen Bibliotheken auf.

Diese Arbeit untersucht die derzeitige Katalogisierungssituation in Sachsen-Anhalts hauptamtlich geleiteten Öffentlichen Bibliotheken und geht der Fragestellung nach, inwieweit die derzeitigen technischen Möglichkeiten genutzt werden, um Katalogisate durch Fremddaten zu beziehen. Dazu werden die Auswertungen einer Umfrage sowie von Expertengesprächen herangezogen. So kann ein realistisches Bild der Fremddatennutzung in der Katalogisierungspraxis in Öffentlichen Bibliotheken des Landes Sachsen-Anhalt gezeichnet werden.

Die Arbeit stellt Lösungsansätze vor, die geeignet erscheinen, um in Öffentlichen Bibliotheken unter den derzeitigen Verhältnissen Katalogisierungsvorgänge durch den Einsatz von Fremddaten effizienter zu gestalten.

In der bibliothekarischen Fachliteratur ist bereits Ende der 1980er und Anfang der 1990er Jahre von Fremddatenübernahme die Rede - auch für öffentliche Bibliotheken. In einem damaligen Artikel in der Zeitschrift ,Bibliothek - Forschung und Praxis ${ }^{22}$ wird eine Situation der FremddatenÜbernahmemöglichkeiten beschrieben, die heute, 2019, in einigen Punkten noch nahezu unverändert besteht. Schon 1988 wird die ,Fremddatenübernahme in Öffentlichen Bibliotheken ${ }^{3}$ ausführlich dargestellt. In den letzten 25 Jahren fehlen Publikationen zum Thema Katalogisierung und Fremddatenübernahme in Öffentlichen Bibliotheken fast vollständig, so dass „zum heutigen Zeitpunkt keine übergreifenden Informationen zur praktischen Vorgehensweise der Katalogisierung in diesen Bibliotheken vorliegen. ${ }^{4}$

Was ist die Ursache, und wie sieht 2018/2019 die Praxis in Öffentlichen Bibliotheken aus? Ausgangspunkt der Masterarbeit ist die Fragestellung, inwieweit die Bibliotheken den Sprung

\footnotetext{
${ }^{1}$ Gantert 2016, S. 175.

2 Vgl. Baum 1992, S. 376-418.

${ }^{3}$ Vgl. Nolte 1988 , S. 151-160.

${ }^{4}$ Woppowa 2018, S. 10.
} 
vom analogen Zettelkatalog zum elektronischen Katalog und von der eigenen manuellen Titelaufnahme zur möglichst automatisierten Fremddatenübernahme geschafft haben. Es stellt sich die Frage: ,Kann die einzelne Bibliothek dies realisieren oder bringt die dezentrale Anbindung an die jeweilige Kommune und damit einhergehend die jeweilige finanzielle Situation hier große Unterschiede hervor?"

Seit der Einführung der elektronischen Datenverarbeitung in Bibliotheken haben sich sowohl die technischen Möglichkeiten als auch das Spektrum des Datenangebots und die Übernahmemöglichkeiten von Katalogisaten oder Metadaten erheblich verändert. ${ }^{5}$ Mit dem neuen Regelwerk Resource Description and Access (RDA), dem Umstieg der bibliographischen Datenformate von MAB2 auf MARC21, neuen Angeboten zur kostenfreien Datenübernahme und zahlreichen Möglichkeiten der Kataloganreicherung veränderte sich die Katalogisierungssituation in den letzten Jahren von Grund auf.

Wissenschaftliche Bibliotheken haben sich in Deutschland seit den 1970er Jahren in sechs regionalen Bibliotheksverbünden ${ }^{6}$ zusammengeschlossen (siehe auch Abb. 2). Sie ermöglichen neben der kooperativen Katalogisierung durch die Standortnachweise in den jeweiligen Verbundkatalogen ebenfalls den überregionalen Leihverkehr. Darüber hinaus bieten sie Fortbildungsveranstaltungen an und erfüllen Planungs- und Entwicklungsaufgaben. ${ }^{7}$ Das Land Sachsen-Anhalt ist 1996 dem Gemeinsamen Bibliotheksverbund (GBV) ${ }^{8}$ beigetreten - einem PICA-Verbund ${ }^{9}$, der eine gemeinsame Katalogisierung mittels festgelegter Verbundrichtlinien ermöglicht ${ }^{10}$. Öffentliche Bibliotheken nutzen jedoch in den meisten Fällen ein lokales Bibliotheksmanagementsystem, das nicht auf PICA basiert. Sie können somit nicht an den landes- oder verbundweiten Angeboten partizipieren.

Die Verbundzentrale des GBV bietet Öffentlichen Bibliotheken die Möglichkeit, ihre Bestandsdaten in den Verbundkatalog der Öffentlichen Bibliotheken (ÖVK) ${ }^{11} z u$ importieren und somit einem größeren Nutzerkreis zu präsentieren. Der ÖVK wird von den Bundesländern Niedersachsen, Sachsen-Anhalt und Thüringen genutzt. Der gemeinsam zur Verfügung stehende

\footnotetext{
${ }^{5}$ Gantert 2016, S. 175.

${ }^{6} 2019$ werden der GBV und der SWB ihre Kataloge zum neuen Katalog K10plus fusionieren; vgl. https://www.bszgbv.de/services/k10plus/ (10.02.2019).

${ }^{7}$ Gantert 2016, S. 43.

${ }^{8}$ https://www.gbv.de/VerbundVerbundzentrale des GBV o.J.d (10.02.2019).

${ }^{9}$ Das PICA-Format ist das interne Datenformat zur Katalogisierung im GBV, das in PICA3 und PICA+ unterteilt ist; Vgl. https://verbundwiki.gbv.de/display/VZG/PICA-Format (10.02.2019).

${ }^{10}$ https://www.gbv.de/bibliotheken/verbundbibliotheken/02Verbund/01Erschliessung/

02RichtlinienVerbundzentrale des GBV o.J.b (29.12.2018).

${ }^{11}$ https://gso.gbv.de/DB=1.126/LNG=DU/ (03.10.2018).
} 
Datenpool wird vor allem zur Fernleihabwicklung eingesetzt. Das Einspielen von Bibliotheksdatensätzen erfolgt nicht automatisiert und findet daher lediglich in großen zeitlichen Abständen statt. In dieser Organisationsform ist der Datenbestand des ÖVK als Fremddatenpool für eine aktuelle Titelaufnahme in der Regel nicht nutzbar. Die bestehende Situation führt seit einigen Jahren dazu, dass große Bibliotheken im Einzugsgebiet des GBV aus dem für Öffentliche Bibliotheken konzipierten Verbundkatalog (ÖVK) in den Katalog der Wissenschaftlichen Bibliotheken (GVK) gewechselt sind (Stadtbibliotheken Braunschweig, Bremen ${ }^{12}$ und Hannover). Dieser Wechsel bringt für die Bibliotheken den Vorteil der OnlineVerbundkatalogisierung.

Die Öffentlichen Bibliotheken des Bundeslandes Sachsen-Anhalt sind diesen Weg bisher nicht gegangen. Den Bibliotheken bleibt die Möglichkeit des Einkaufs von Katalogdaten, der Datenübernahme z.B. bei der DNB ${ }^{13}$, den Bibliotheksverbünden oder über das ZACK-Gateway ${ }^{14}$, die zeitaufwändige manuelle Katalogaufnahme, oder die Anwendung von Mischformen der Titelaufnahmen durch Kopieren und Einfügen. Um dies belegen zu können, wurde eine Umfrage unter den hauptamtlichen sachsen-anhaltinischen Öffentlichen Bibliotheken durchgeführt mit dem Ziel, den derzeitigen Stand zu ermitteln und die Ursachen für die Situation aufzulisten.

Die Katalogisierungspraxis in Öffentlichen Bibliotheken wurde bisher kaum untersucht und soll in dieser Arbeit speziell für die in Sachsen-Anhalt bestehenden Varianten recherchiert, analysiert und ausgewertet werden. Die Begrenzung auf ein Bundesland ist bewusst gewählt worden, da sich bei der Entwicklungs- und Gestaltungsmöglichkeit von Bibliotheken die Rahmenbedingungen in den Bundesländern zu sehr unterscheiden, z.B.:

- die politischen Verhältnisse in Bezug auf Reformen und finanzielle Zuwendungen

- die unterschiedliche Anbindung an eine Verbunddatenbank

- das Vorhandensein oder Nichtexistieren eines Bibliotheksgesetzes mit den damit verbundenen Vorgaben, Förderungen oder Absicherungen für Bibliotheken.

In dieser Arbeit soll der derzeitige Aufwand der Katalogisierung ${ }^{15}$ in Sachsen-Anhalt dargelegt sowie eine Übersicht erstellt werden, auf welchem Weg eine Fremddatenübernahme für die eingesetzten Systeme möglich ist.

\footnotetext{
12 https://www.gbv.de/news/neues-aus-der-verbundzentrale/2013/stadtbibliothekVerbundzentrale des GBV o.J.c (10.02.2019).

${ }^{13}$ http://www.dnb.de/DE/Service/DigitaleDienste/digitaledienste node.html (10.02.2019).

${ }^{14}$ https://www.z3950.de/zackSchneider 1999 (10.02.2019).

${ }^{15}$ Der Vorgang der Aufnahme der Metadaten in die jeweilige Bibliotheksdatenbank.
} 
Die inhaltlichen Aspekte der Themenkomplexe RDA-Umstieg und neues Datenformat MARC21 werden im Rahmen dieser Masterarbeit nicht untersucht.

Zunächst erfolgt ein Überblick über die derzeitige Situation der Öffentlichen Bibliotheken in Sachsen-Anhalt. Diese wurde durch mehrere Kreisgebietsreformen beeinflusst, durch die sich die Zuordnung der Zuständigkeiten und Einzugsbereiche der einzelnen Bibliotheken veränderte. Dies führte zu gravierenden Unterschieden der Anzahl der zu betreuenden Einwohner. Hierzu wird die Zahl des zur Verfügung stehenden Personals in einem statistischen Vergleich auf Basis der DBS-Daten dargelegt. Ein geschichtlicher Abriss und die Funktion des ÖVK als der Katalog der Öffentlichen Bibliotheken komplettiert die Situationsdarstellung.

Weiterhin wird auf die derzeitige Katalogisierungssituation und die verwendeten Standards eingegangen. In diese Artikel fließen die Ergebnisse der im Rahmen dieser Arbeit durchgeführten Online-Umfrage schon mit ein, deren detaillierte Auswertung einen großen Teil der Arbeit einnimmt.

Im Anschluss werden die durch Experteninterviews ermittelten Methoden der Fremddatenübernahme der im Bundesland eingesetzten Bibliotheksmanagementsysteme dargestellt, sowie exemplarische Anleitungen erstellt bzw. vorhandene im Anhang beigefügt. 


\section{2. Öffentliche Bibliotheken in Sachsen-Anhalt}

In diesem Kapitel wird die Institution Öffentliche Bibliothek in ihrer Funktion und Bestimmung beschrieben, um das Thema Katalogisierung als Forschungsgegenstand der vorliegenden Arbeit in einen inhaltlichen und regionalen Rahmen einzuordnen.

Begonnen wird mit der Struktur der deutschen Bibliothekslandschaft, insbesondere der in Sachsen-Anhalt. Im Anschluss wird der Unterschied zwischen Öffentlichen und Wissenschaftlichen Bibliotheken aufgezeigt. Ein Vergleich der Jahre 2007, 2012 und 2017 legt die derzeitige Situation detailliert dar.

Weiterhin wird der ÖVK als gemeinsamer Katalog der Öffentlichen Bibliotheken in SachsenAnhalt erläutert.

\subsection{Begriffsbestimmung und Abgrenzung}

Öffentliche und Wissenschaftliche Bibliotheken sind Teil des Netzes der deutschen und internationalen Informationsversorgung. Wie im Strategiekonzept Bibliotheken 2007 beschrieben, erfüllen sie durch Maßnahmen der Leseförderung und zielgruppenorientierte Angebote einen bildungspolitischen Auftrag bei der Entwicklung von Lese- und Medienkompetenz. Sie bieten Orientierung im Alltag, im Umgang mit Informationen sowie zur kreativen Freizeitgestaltung und unterstützen bei der kompetenten Nutzung des Internets, und bieten z.T. die Möglichkeit, das Internet und weitere elektronische Quellen überhaupt nutzen zu können. Sie beschaffen, erschließen, archivieren und erhalten gedruckte wie elektronische Literatur- und Informationsbestände sowie Bestände weiterer Medientypen ${ }^{16}$ und tragen damit als neutraler Anbieter und Vermittler von Informationen zur Erfüllung des Grundrechts auf freien Zugang zur Information und zur Chancengleichheit bei. ${ }^{17}$

Verbreitet ist in Deutschland die Aufteilung in die Sparten Öffentliche Bibliotheken (mitunter auch Büchereien genannt) und Wissenschaftliche Bibliotheken. ${ }^{18}$

Öffentliche Bibliotheken dienen vorwiegend „der allgemeinen Information, der politischen und beruflichen Bildung sowie der Unterhaltung (und stellen) ihre Bestände der gesamten Öffentlichkeit ohne Einschränkung zur Verfügung. ${ }^{\text {19 }}$ Sie werden von Gemeinden, Städten oder Landkreisen finanziert.

\footnotetext{
16 U.a.: Musik-CDs, Hörbücher, Konsolen- und Gesellschaftsspiele.

${ }^{17}$ Vgl. Beger 2004, S. 10-11.

18 Gantert 2016, S. 9.

${ }^{19}$ Ebd.
} 
Wissenschaftliche Bibliotheken dienen „vor allem dem wissenschaftlichen Studium und der Forschung. ${ }^{20}$ Die Finanzierung erfolgt ebenfalls aus öffentlicher Hand ${ }^{21}$.

Die Unterteilung dieser beiden Sparten ist nicht immer eindeutig. Auch eine Wissenschaftliche Bibliothek ist i.d.R. öffentlich zugänglich, ebenso wie eine Öffentliche Bibliothek auch wissenschaftliche Medien zur Verfügung stellen kann. Bibliotheken der Sparte Wissenschaftliche Bibliothek sind nicht Thema dieser Masterarbeit. Betrachtet werden nur die als Öffentliche Bibliothek getypten Bibliotheken, die sich durch einen freien Zugang für alle Bevölkerungsschichten definieren.

Eine Strukturierung der deutschen Bibliotheken liegt in verschiedenen Ansätzen vor. Neben der Unterteilung in Wissenschaftliche und Öffentliche Bibliotheken werden Bibliotheken nach dem zu erbringenden Versorgungsgrad des zu betreuenden Bereiches untergliedert. Um den Literatur- und Informationsbedarf zu decken, wurde eine heute noch gültige Grundeinteilung im „Bibliotheksplan '73“22 festgelegt und in dem Positionspapier „Bibliotheken '93“23 übernommen. Darin festgeschrieben ist eine Einteilung in 4 Funktionsstufen.

Bibliotheken der Stufe 1 sind Einrichtungen, die den Grundbedarf der Bevölkerung abdecken. Hierzu zählen kleinere und mittlere Öffentliche Bibliotheken, sowie Zweigstellen von Großstadtbibliotheken. In Sachsen-Anhalt zählen dazu 72 der in der Umfrage betrachteten Bibliotheken, sowie insgesamt 12 Zweigstellen der 3 Großstadtbibliotheken.

Zur 2. Stufe zählen die Zentralbibliotheken der 3 Großstadtbibliotheken Dessau, Halle und Magdeburg. Sie haben mit ihrem Angebot einen gehobenen Bedarf abzudecken.

Die 3. und 4. Stufe sind den Bibliotheken vorbehalten, die den spezialisierten Bedarf bzw. den hochspezialisierten Bedarf abdecken. Für Öffentliche Bibliotheken wäre dies eine Zentralbibliothek einer Stadt mit mindestens 400.000 Einwohnern, die es in Sachsen-Anhalt aber nicht gibt.

Die in dieser Arbeit betrachteten Bibliotheken gehören also bis auf die Zentralbibliotheken der Städte Dessau, Halle und Magdeburg der Stufe 1 an.

Eine weitere Einteilung der Bibliotheken nimmt der Deutsche Bibliotheksverband (DBV) vor. Dieser Institutionsverband ist ein Zusammenschluss von Öffentlichen und Wissenschaftlichen Bibliotheken, dessen Anliegen es ist, "die Wirkung von Bibliotheken in Kultur und Bildung sichtbar zu machen und ihre Rolle in der Gesellschaft zu stärken. “24 Der DBV gliedert sich in 16

\footnotetext{
${ }^{20}$ Gantert 2016, S. 9.

${ }^{21}$ Ausnahmen sind z. B. kirchliche Bibliotheken oder Bibliotheken privater Hochschulen.

22 Bibliotheksplan 19731973.

${ }^{23}$ Bibliotheken '93 1994.

${ }^{24}$ https://www.bibliotheksverband.de/dbv/ (13.1.2019).
} 
Landesverbände und 9 Sektionen. Vier Sektionen sind dabei den Öffentlichen Bibliotheken vorbehalten. ${ }^{25}$ Ein Vergleich der Typisierungen zeigt auf, dass die Sektion 1 des DBV der 3. Stufe aus ,Bibliotheken '93' entspricht. ${ }^{26}$ Der Sektion 2 sind die 3 Großstadtbibliotheken zugeordnet wobei Dessau einen Sonderstatus genießt, da die Einwohnerzahl nicht mehr der vorgegebenen Größe von 100.000 Einwohnern entspricht. Wittenberg ist in der Sektion 3A und 57 Bibliotheken sind in der Sektion 3B. ${ }^{27}$ gelistet. Der DBV Sachsen-Anhalt führt zentrale Weiterbildungsveranstaltungen durch und ist durch die Vergabe von Fördermitteln eine direkte Unterstützung der Bibliotheken.

Der Landesentwicklungsplan für das Land Sachsen-Anhalt aus dem Jahr 2010 wiederum legt in der Beikarte $1^{28}$ die drei Städte Dessau, Halle und Magdeburg als Oberzentren fest. Bibliotheken in Oberzentren erhalten eine in der „Kulturförderrichtlinie Sachsen-Anhalt"29 festgeschriebene Förderung für den Kauf von Medieneinheiten.

Sachsen-Anhalt besteht aus 3 kreisfreien Städten und 11 Landkreisen. ${ }^{30}$ Die Landesfachstelle für Öffentliche Bibliotheken des Landes Sachsen-Anhalt verzeichnet für das Bundesland 75 Bibliotheken $^{31}$, die den Kriterien der in dieser Arbeit betrachteten hauptamtlich geführten Öffentlichen Bibliotheken entsprechen. Reine Schulbibliotheken werden in die Umfrage nicht einbezogen. Die kleinste in der Umfrage erfasste Bibliothek mit hauptamtlichem Personal versorgt knapp 2.000 Einwohner, zwei der drei Großstadtbibliotheken stehen für jeweils ca. 240.000 Einwohner zur Verfügung. Dieser Spanne im Betreuungsschlüssel ist eine in dieser Arbeit vorgenommene Unterteilung in Einwohnergrößengruppen geschuldet, mit dem Ziel, eine statistische Auswertung auf eine vergleichbare Basis zu stellen. Die Situation, der in der Arbeit hauptsächlich betrachteten Arbeitsbereiche der Katalogisierung ist in einer One Person Library (OPL) anders zu bewerten als in Bibliotheken, die für diesen Arbeitsbereich spezialisierte Mitarbeiterinnen ${ }^{32}$ einsetzen.

Öffentliche Bibliotheken sind nach dem sachsen-anhaltinischen Bibliotheksgesetz definiert als „Teil des Bildungssystems und dienen der schulischen, beruflichen, allgemeinen und kulturellen Bildung, der Vermittlung von Medien- und Informationskompetenz sowie der Pflege von

\footnotetext{
${ }^{25}$ https://www.bibliotheksverband.de/fachgruppen/sektionen.html (05.01.2019).

${ }^{26}$ Eine Stadt mit dieser Einwohnerzahl ist in Sachsen-Anhalt nicht vorhanden.

${ }^{27}$ https://www.bibliotheksverband.de/landesverbaende/sachsen-anhalt/mitglieder.html (13.1.2019).

${ }^{28}$ Ministerium für Landesentwicklung und Verkehr des Landes Sachsen-Anhalt 2011 http://www.landesrecht.sachsen-anhalt.de/jportal/docs/anlage/Ir/st/a4f7f9e3-645f-4cf3-9543-bc9049df48b6st230.10+2011+160+p+anhang_1.pdf (03.03.2019).

29 http://www.foerderdatenbank.de/Foerder-DB/Navigation/Foerderrecherche/suche.html?get= 056c1600964814c7ac8c99bf030cba03;views;document\&doc=10722\&typ=RL (10.01.2019).

${ }^{30}$ Statistisches Landesamt Sachsen-Anhalt 2018.

31 https://Ivwa.sachsen-anhalt.de/das-Ivwa/kultur-denkmalschutz/landesfachstelle-fuer-oeffentlichebibliotheken/bibliotheken-in-sachsen-anhalt/ (03.03.2019).

32 Die weibliche Form steht in dieser Arbeit für alle Geschlechter, da der größte Teil des Bibliothekspersonals dem weiblichen Geschlecht zuzurechnen ist.
} 
Sprache und Literatur. Sie unterstützen in besonderer Weise die Leseförderung bei Kindern und Jugendlichen und tragen insbesondere mit der Bereitstellung fremdsprachiger Literatur und der Durchführung interkultureller Veranstaltungen zur interkulturellen Bildung bei.“ ( $§ 3$ Abs. 2 BibIG LSA $)^{33}$

Die Öffentlichen Bibliotheken des Bundeslandes werden durch die Landesfachstelle für Öffentliche Bibliotheken des Landes Sachsen-Anhalt im Landesverwaltungsamt ${ }^{34}$ fachlich begleitet. Die Landesfachstelle veranstaltet Fortbildungsveranstaltungen und ist beratend in spezifischen Bibliotheksbelangen tätig, u.a. zu den Themen Bestandsaufbau und -ausstattung sowie DV-Anwendungen. Ihr Rechtsanspruch ist mit den konkret festgelegten Aufgaben und Verantwortlichkeiten im Bibliotheksgesetz Sachsen-Anhalt ${ }^{35}$ verankert.

Seit einigen Jahren fungieren Öffentliche Bibliotheken in zunehmendem Maße als ein sogenannter ,dritter Ort'. Gemeint sind frei zugängliche Räumlichkeiten, in denen man lernen, lesen oder spielen kann, in denen Informationsveranstaltungen und Schulungen stattfinden. Dieser Begriff wurde 1989 von dem amerikanischen Soziologen Ray Oldenburg geprägt. ${ }^{36}$ Die meisten seiner Beschreibungen eines solchen Ortes sind auf Bibliotheken zutreffend, weshalb der Begriff in den Sprachgebrauch der Bibliotheken übergegangen ist, um eine Bezeichnung dafür zu finden, was Bibliotheken heutzutage sind und darstellen.

„Unsere dritte[n] Orte sollen fair, inspirierend, informell und persönlich sein.“ Aat Vos ${ }^{37}$

Prägende Beispiele der Umsetzung dieser neuen Herangehensweise sind die Stadtbibliothek Dokk1 in Aarhus/Dänemark ${ }^{38}$ sowie die 2018 neu eröffnete Stadtteilbibliothek ,Oodi' im finnischen Helsinki ${ }^{39}$.

\section{2. ÖVK - der Katalog der Öffentlichen Bibliotheken}

Das Land Sachsen-Anhalt legt in seinem Bibliotheksgesetz fest, dass die Vernetzung von Öffentlichen Bibliotheken durch das Land gefördert wird. Dies ist in der Kulturförderrichtlinie explizit definiert: „Die inhaltlichen Schwerpunkte der Förderung [...] sind: Projekte, die der Entwicklung effektiver Strukturen der bibliotheksmäßigen Versorgung dienen, insbesondere unter Beachtung einer regionalen oder spartenübergreifenden Vernetzung“ ${ }^{40}$

\footnotetext{
33 Online verfügbar unter: http://www.landesrecht.sachsenanhalt.de/jportal/?quelle=jlink\&query=BiblG+ST\&psml=bssahprod.psml\&max=true\&aiz=true (11.02.2019)

${ }^{34} \mathrm{https}$ ///lvwa.sachsen-anhalt.de/das-Ivwa/kultur-denkmalschutz/landesfachstelle-fuer-oeffentliche-bibliotheken/ (11.02.2019).

35 http://www.landesrecht.sachsen-

anhalt.de/jportal/?quelle=jlink\&query=BiblG+ST\&psml=bssahprod.psml\&max=true\&aiz=true $(11.02,2019)$.

${ }^{36}$ Oldenburg 1989.

37 https://aatvos.com/warum-bibliotheken-ein-dritter-ort-fur-alle-werden-sollten/ (11.02.2019).

${ }^{38}$ https://www.visitaarhus.de/de/dokk1-gdk1077504 (11.02.2019).

${ }^{39} \mathrm{https}: / /$ finland.fi/de/leben-amp-gesellschaft/helsinki-investiert-in-eine-bibliothek-die-ihr-wesen-neu-erfindet/ (11.02.2019).

${ }^{40}$ Landesverwaltungsamt Sachsen-Anhalt Erl. der StK vom 2017, Punkt 2.4 Abs. m, aa.
} 
Mit Gründung des GBV 1996 ist das Land Sachsen-Anhalt diesem Bibliotheksverbund beigetreten und finanziert die Mitgliedschaft laut Verwaltungsabkommen ${ }^{41}$. Seit 2004 bietet der GBV für Öffentliche Bibliotheken eine separate Datenbank zur Speicherung ihrer Katalogdaten im PICA-Format an. Der ÖVK ist der „frei zugängliche Verbundkatalog der Öffentlichen Bibliotheken im GBV [, er] enthält über 4,1 Mio. Titel- und über 6,9 Mio. Besitznachweise von mehr als 150 Bibliotheken“42 aus den Ländern Thüringen, Sachsen-Anhalt und Niedersachsen.

Die Teilnahme am ÖVK ist für Bibliotheken aus Sachsen-Anhalt mit keinen weiteren Kosten für die jeweilige Bibliothek verbunden. Eine Teilnahme ist per formlosem Antrag ${ }^{43}$ an die Verbundzentrale des GBV (VZG) möglich. Voraussetzung ist eine Sigelnummer der deutschen ISIL-Agentur und Sigelstelle.

Der ÖVK fungiert als zentraler überregionaler Standortnachweis. Durch separate Sichten kann die Ansicht auf Bundesländer, Landkreise bis zur Auswahl einzelner Bibliotheken eingeschränkt und somit separat angezeigt werden.

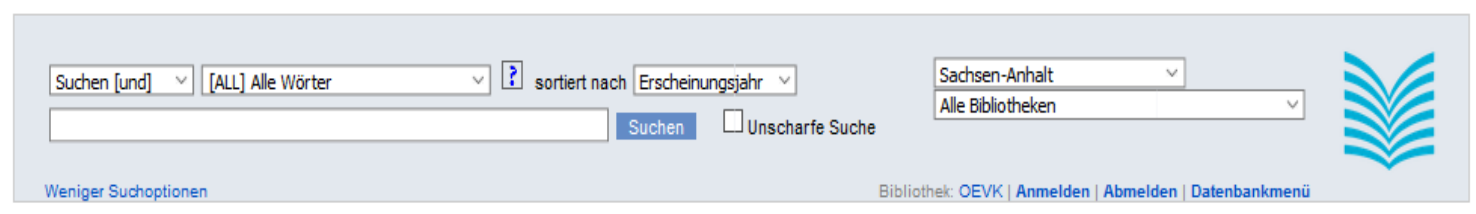

Abb.: 1 Darstellung der regionalen Suchmöglichkeit - Sicht für Sachsen-Anhalt

Seine Hauptfunktion besteht derzeit in der Durchführung der gebenden und nehmenden Fernleihe, die über ein Web-Frontend durchgeführt wird. Bibliotheken können Lieferprofile und Verfügbarkeitsfristen festlegen ${ }^{44}$. Eine Z39.50- sowie eine SRU-Schnittstelle ermöglichen den Zugriff auf die Datenbanken des GVK und des ÖVK.

Es findet keine zentrale oder kooperative Katalogisierung direkt in der ÖVK-Datenbank statt. Jede Bibliothek erfasst die Katalogisate im lokalen Bibliothekssystem und übermittelt Ihre Katalogdaten in regelmäßigen Abständen an den ÖVK.

Der ÖVK ist in der jetzigen Form als Datenquelle für eine Fremddatenübernahme wenig geeignet, da aufgrund der langen Import-Intervalle kaum aktuelle Daten vorhanden sind, die von Öffentlichen Bibliotheken für die Katalogisierung ihrer Neuanschaffungen nachgenutzt werden können. Als Grund für eine Nichtteilnahme am ÖVK wurde dieser zeitliche Verzug von den Umfrageteilnehmern jedoch nicht ausgewählt.

\footnotetext{
${ }^{41}$ Verbundzentrale des GBV 1996, §9.

42 Verbundzentrale des GBV 2019, Siehe: https://gso.gbv.de (10.03.2019).

${ }^{43}$ Online-Antrag für Bibliothekssigel: http://sigel.staatsbibliothek-berlin.de/de/beantragung/.

${ }^{44}$ Lange 2018, F. 4.
} 
Ein separater aktueller Katalog für Öffentliche Bibliotheken aus der Region hätte für eine Fremddatenübernahme den Vorteil, dass die Klassifikation und auch die Schlagwortvergabe durch die politisch ähnliche Situation und Geschichte oft identisch sind. Übernahmen müssten nicht aus einer Vielzahl von Treffern in verschiedenen Bibliotheksverbünden herausgefiltert werden. Die Auswahl eines geeigneten Treffers in größeren Datenbanken erscheint mehreren Teilnehmenden oft zeitaufwändiger, als ein eigenes Katalogisat zu erstellen. Auch wird eine zielgerichtete Suche vor der Fremddatenübernahme als zeitraubend empfunden. Es stellte sich die Frage, welche Datenbank für die Öffentlichen Bibliotheken in Sachsen-Anhalt als Fremddatenpool favorisiert wird.

Von 39 Bibliotheksleitungen gaben 2/3 (27 Bibliotheken) an, dass sie Mitglied im ÖVK sind. Die Nichtteilnehmer geben zu 33\% (3 Bibliotheken) mangelndes Know-how oder fehlende Mittel und technische Voraussetzungen als Grund an. $44 \%$ (4 Bibliotheken) geben an, dass sie sich damit noch nicht beschäftigt haben. 2 Bibliotheken (22\%) geben keine Gründe für eine Nichtteilnahme an. 2 Bibliotheken umgingen generell die Antwort mit ,keine Angabe'.

Eine Bibliothek gibt an, dass ihre Daten im BRISEOpac präsentiert werden. BRISE ist ein seit dem Jahr 2000 betriebenes Medienportal, das den Bibliotheken softwareunabhängig die Teilnahme an einer virtuellen Bibliothek ermöglicht ${ }^{45}$.

Auf der Grundlage des Verwaltungsabkommens mit dem Land Sachsen-Anhalt wurde der GBV ab 2004 beauftragt, im Anschluss an das BRISE-Projekt einen Verbundkatalog für Öffentliche Bibliotheken des Landes Sachsen-Anhalt aufzubauen und zu betreiben.

Der GBV plant im Jahr 2019 im Zusammenhang mit dem Start von K10Plus eine Neufassung der Datenbankstrukturen im ÖVK mit einem „überarbeiteten Importverfahren für mehr Automatisierung und schnellere Updates ${ }^{\prime 46}$. Mittels Master Record Model (MRM) ${ }^{47}$ wird ein neues Abgleichverfahren implementiert, welches das vorher genutzte Match \& Merge $(M+M)^{48}$ Verfahren ablöst. Eine erhöhte Aktualisierungsrate sowie die Möglichkeit für DifferenzLieferungen kann die Aktualität des ÖVK deutlich erhöhen. Eine Fernleihe für aktuellste Medien aber auch die Möglichkeit, den ÖVK als Fremddatenquelle für aktuelle Medien nutzen zu können, wären erstmals möglich. (siehe Fazit)

\footnotetext{
${ }^{45}$ Siehe: http://www.herrmann-pflug.de/html/brise.htmHerrmann [und] Pflug GbR.

${ }^{46}$ Lange 2018, F.8.

${ }^{47}$ Ein primärer Datensatz wird als Master angelegt - diesem werden die individuellen Datensätze der einzelnen Bibliotheken („Slave-Records“) zugeordnet. Siehe: Sommerschuh 2014, S. 3.

${ }^{48}$ Ein über festgelegte Regeln zur Erkennung von Dubletten angewendetes Abgleichverfahren bei der

Zusammenführung von Daten.
} 
Aktuelle Situation der Öffentlichen Bibliotheken

in Sachsen-Anhalt

\section{Aktuelle Situation der Öffentlichen Bibliotheken in Sachsen-Anhalt}

Dieses Kapitel erklärt die Aufteilung der Öffentlichen Bibliotheken in die Gebietsstrukturen der jeweiligen Städte, Gemeinden und Landkreise und stellt anhand einer Statistikauswertung die personellen Ressourcen in Bezug zu vorliegenden Berechnungsgrundlagen dar.

\subsection{Struktur und Gliederung der Institutionen}

Die politische Gebietsstruktur in Sachsen-Anhalt wurde durch zwei Kreisgebietsreformen ${ }^{49}$ verändert. Diese Reformen haben naturgemäß Einfluss auf die in den Gemeinden und Landkreisen angesiedelten kulturellen Bildungseinrichtungen. Die Bildung von neuen Einheitsgemeinden mit über 10.000 Einwohnern führte bis jetzt nicht nachweislich zu einer Neustrukturierung der Bibliothekslandschaft in den betreffenden Ortschaften. So stehen der Bevölkerung in den 31 Gemeinden mit 10.000 - 20.000 Einwohnern ${ }^{50}$ nur 22 hauptamtlich geleitete Bibliotheken zur Verfügung. Die Verteilung der Öffentlichen Bibliotheken in SachsenAnhalt nach Kreisen und Einwohnergrößen zeigt in Tabelle 1, dass jede Gemeinde ab 20.000 Einwohnern über eine hauptamtlich geleitete Bibliothek verfügt.

Die Anzahl der Gemeinden unter 10.000 Einwohnern liegt bei weit über 100. Diese Zahl kann in diesem Vergleich nicht genutzt werden, da keine statistischen Werte für nebenberuflich geführte Bibliotheken vorliegen. Die betreffenden Gesamtwerte sind in Tabelle 1 daher in Klammern dargestellt.

Die Bezeichnungen der Bibliotheken zeigen i.d.R. die unterschiedlichen Zuständigkeiten auf. Mit 77\% liegen 57 Bibliotheken in der Zuständigkeit der jeweiligen Stadt bzw. Gemeinde und haben die Bezeichnung Stadt- oder Gemeindebibliothek bzw. -bücherei. Überregionale Zuständigkeit haben 12 Bibliotheken (16\%). Vereinzelt sind Bibliotheken an Volkshochschulen, TouristInformationen und Schulen angebunden.

\footnotetext{
${ }^{49}$ Kreisgebietsreform 1994 mit Reduzierung von 37 auf 21 und 2007 mit Reduzierung auf 11 Landkreise.

${ }^{50}$ Quelle: https://statistik.sachsen-anhalt.de/fileadmin/Bibliothek/Landesaemter/StaLa/startseite/Themen/ Bevoelkerung/Berichte/Bevoelkerungsstand/6A102_02_17-A.pdf S. 6.
} 
Tab.: 1 Anzahl der hauptamtlich geführten Öffentlichen Bibliotheken in Sachsen-Anhalt nach Kreisen und Einwohnergrößen (Stand 2018) ${ }^{51}$

\begin{tabular}{|l|c|c|c|c|c|c|c|}
\hline Landkreise & Einwohner & & & & & \\
\hline Anhalt-Bitterfeld & & & & & & & \\
\hline Börde & 3 & 1 & 2 & 1 & 0 & 0 & 7 \\
\hline Burgenlandkreis & 4 & 4 & 0 & 0 & 0 & 0 & 8 \\
\hline Harz & 5 & 0 & 1 & 1 & 1 & 0 & 8 \\
\hline Jerichower Land & 2 & 2 & 2 & 1 & 1 & 0 & 8 \\
\hline Mansfeld-Südharz & 0 & 2 & 1 & 0 & 0 & 0 & 3 \\
\hline Altmarkkreis Salzwedel & 4 & 1 & 3 & 0 & 0 & 0 & 4 \\
\hline Stendal & 3 & 1 & 1 & 1 & 0 & 0 & 7 \\
\hline Saalekreis & 2 & 7 & 0 & 1 & 0 & 0 & 10 \\
\hline Salzlandkreis & 2 & 0 & 2 & 2 & 0 & 0 & 6 \\
\hline Wittenberg & 3 & 3 & 0 & 0 & 1 & 0 & 7 \\
\hline Dessau & 0 & 0 & 0 & 0 & 0 & 1 & 1 \\
\hline Halle & 0 & 0 & 0 & 0 & 0 & 1 & 1 \\
\hline Magdeburg & 0 & 0 & 0 & 0 & 0 & 1 & 1 \\
\hline gesamt & $(28)^{52}$ & 22 & 12 & 7 & 3 & 3 & 47 \\
\hline Anzahl der Gemeinden & $(163)$ & 31 & 11 & 7 & 3 & 3 & 55 \\
\hline
\end{tabular}

\subsection{Statistischer 10-Jahres-Vergleich auf Grundlage der Deutschen} Bibliotheksstatistik

Um die Entwicklung der Öffentlichen Bibliotheken in Sachsen-Anhalt der letzten Jahre besser nachvollziehen zu können, wurde mittels Sekundärdaten der DBS-Statistik ${ }^{53}$ eine Vergleichsstatistik ${ }^{54}$ der Jahre 2007, 2012 und 2017 erstellt ${ }^{55}$. Neben den drei auszuwertenden Jahren wurden folgende Filter für die Auswahl gesetzt: Öffentliche Bibliotheken, Bundesland Sachsen-Anhalt und hauptamtliche Leitung.

Der Vergleich zeigt, dass die primären Nutzer ${ }^{56}$ in den herausgefilterten Bibliotheken in 10 Jahren um 220.685 angestiegen sind. Die Bevölkerungsstatistik in Sachsen-Anhalt dagegen zeigt

\footnotetext{
${ }^{51}$ Statistisches Landesamt Sachsen-Anhalt 2018, S. 6.; Landesfachstelle für Öffentliche Bibliotheken https://lvwa.sachsen-anhalt.de/das-lvwa/kultur-denkmalschutz/landesfachstelle-fuer-oeffentlichebibliotheken/bibliotheken-in-sachsen-anhalt/ (15.02.2019).

52 Diese Zahlen können wegen der fehlenden Statistik für nebenberufliche Bibliotheken nicht verglichen werden.

53 https://www.hbz-nrw.de/produkte/bibliotheksstatistik (24.02.2019).

${ }^{54}$ Die Tabellen liegen als prüfungsrelevante Dokumentation vor, siehe beiliegende CD.

552007 und 2017 listet die DBS 74 Bibliotheken auf. 2012 nur 73 Bibliotheken. Um vergleichbare Werte zu bekommen, wurde die fehlende Bibliothek (Sandersdorf) für das Jahr 2012 durch Hinzufügen des jeweiligen Mittelwertes der beiden vorhandenen Jahre dieser Bibliothek mit aufgenommen.

${ }^{56} \mathrm{Zu}$ betreuende Einwohner werden in der DBS ,primäre Nutzer' genannt, da sie sich nicht unbedingt nur auf eine bestimmte Gemeinde beziehen.
} 
Aktuelle Situation der Öffentlichen Bibliotheken

in Sachsen-Anhalt

einen Rückgang der Gesamtbevölkerung um 189.391 Einwohner. Ein Grund für diese Diskrepanz liegt in den Kreisgebietsreformen. Viele Bibliotheken sind nun wegen der größeren Einzugsgebiete für mehr Einwohner zuständig. Im Alltag wird eine Gebietsreform sicher nicht dazu beitragen, dass Einwohner in die nächstgrößere Gemeinde fahren, um das Bibliotheksangebot zu nutzen. Aufgrund der zunehmenden digitalen Dienstleistungen ist die Nutzung von Bibliotheksmedien jedoch auch aus entfernt liegenden Orten Realität geworden.

Es wird die Hypothese aufgestellt, dass trotz der angestiegenen Zahl der zu betreuenden Einwohner die Anzahl des Personals nicht im Maß des vorgesehenen Betreuungsschlüssels (Personalstelle/Einwohner) angewachsen ist. Ein Vergleich des Personalschlüssels in den drei Vergleichsjahren zeigt keine Erhöhung, sondern eine deutliche Abnahme des Gesamtpersonals. Gemäß KGST-Gutachten ' $73^{57}$ und Bibliotheken ' $93^{58}$ ist „für eine voll entwickelte Öffentliche Bibliothek (2 Medieneinheiten pro Einwohner, 3 Ausleihen je Medieneinheit pro Jahr) von einem Personalbedarf von einer Personalstelle je 2000 Einwohner auszugehen. ${ }^{49}$ Das Grundlagenwerk ,Bibliotheken und Informationsgesellschaft in Deutschland ${ }^{60}$ nimmt Bezug auf diese Werte, fügt jedoch eine Ergänzung der KGST aus dem Jahr 1994 an, wonach für die Gemeinden der Größenklasse 5 (25.000 - 50.000 Einwohner) eine Reduzierung der Personalausstattung auf 50\% der Werte aus 1973 vorgesehen ist. Eine weitere Empfehlung gab 1991 die ,Bund-LänderArbeitsgruppe Bibliothekswesen ${ }^{61}$ für die neuen Bundesländer. Diese als „politische Reduzierung eines als notwendig anerkannten Personalbedarfs " ${ }^{\prime 62}$ bezeichneten Werte lagen bei 70\% der im KGST-Gutachten empfohlenen Personalstellen. Eine Empfehlung der IFLA ${ }^{63}$ zum Personaleinsatz in Öffentlichen Bibliotheken kommt 2001 auf ähnliche Werte: eine Personalstelle für je 2.500 Einwohner. Im Strategiekonzept Bibliotheken $2007^{64}$ wird zum Personaleinsatz lediglich vermerkt, dass er auf „Erkenntnissen moderner und leistungsorientierter Betriebsführung beruhen" muss. Einschränkende Bemerkungen zum heutigen Einsatz dieser Berechnungstabellen findet man bei Naumann/Umlauf dahingehend, dass die damalige mit der heutigen Situation kaum vergleichbar ist. Als Grund werden der

\footnotetext{
57 Öffentliche Bibliothek (1973), S. 88.

${ }^{58}$ Bibliotheken '93 1994, S. 112; IFLA 2001, S. 8-9.

${ }^{59}$ Naumann/Umlauf, S. 8-9.

60 Plassmann et al. 2011, S. 188.

${ }^{61}$ Bund-Länder-Arbeitsgruppe Bibliothekswesen 1991, S. 24.

62 Naumann/Umlauf, S. 9 Fußnote.

63 IFLA 2001, S. 59.

${ }^{64}$ Beger 2004, S. 21.
} 
Aktuelle Situation der Öffentlichen Bibliotheken

in Sachsen-Anhalt

damalige „geringe Automatisierungsgrad und die große Fertigungstiefe (z.B. kaum Fremddaten bei der Katalogisierung) ${ }^{\prime 65}$ benannt. Hier stellt sich wieder die in der Einleitung gestellte Frage: Welche (Öffentlichen) Bibliotheken mögen sich von dieser Einschätzung angesprochen fühlen? Die auf Grundlage der angegebenen Berechnungsschlüssel in einem Schlüsselzahlverfahren berechneten Werte für Sachsen-Anhalt sind in Abb. 2 dargestellt.

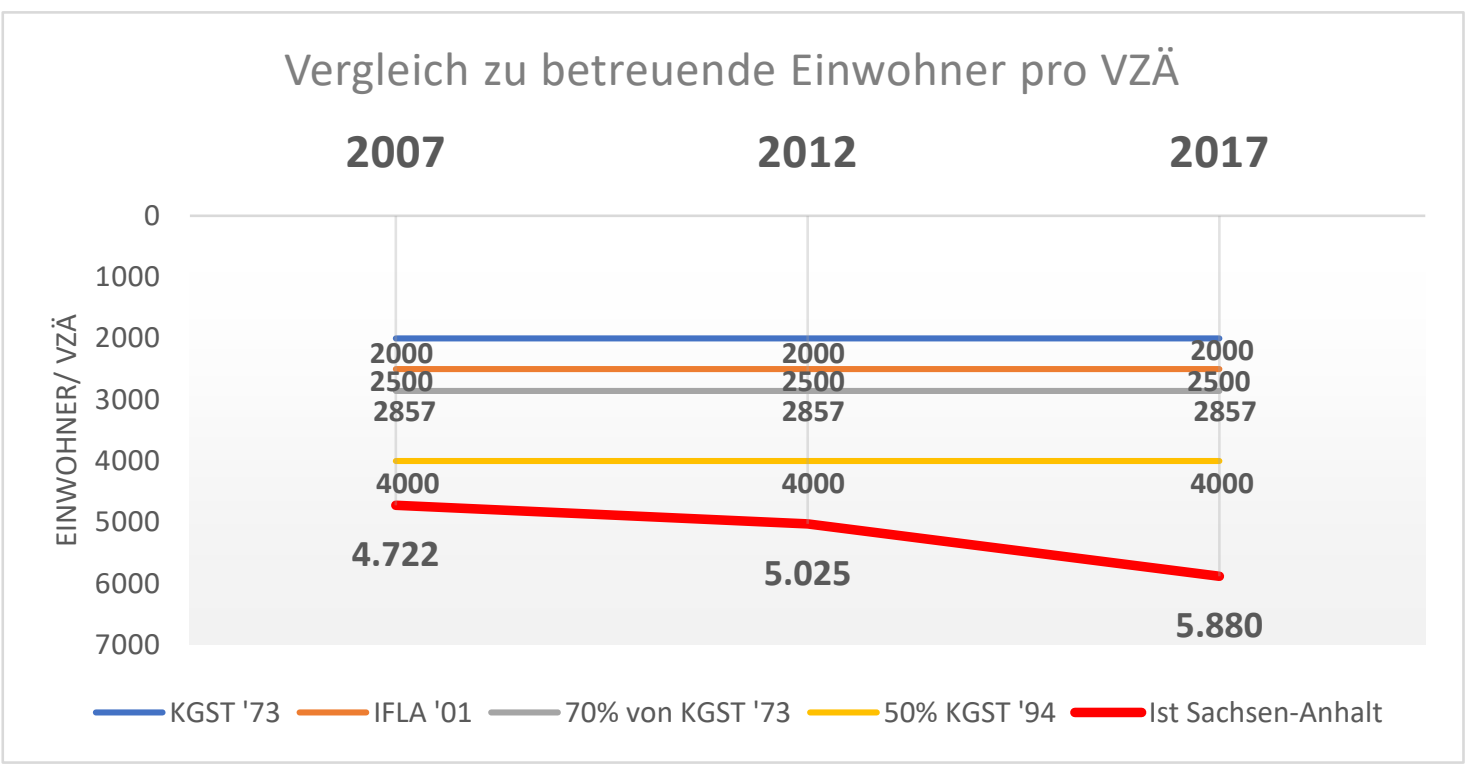

Abb.: 2 Empfohlener Personalbedarf und IST-Zustand in Öffentlichen Bibliotheken Sachsen-Anhalts (zu betreuende Einwohner pro Vollzeitäquivalent - VZÄ)

Die Personalsituation hat sich in Bezug auf die zu betreuende Einwohnerzahl nicht nur verschlechtert, es ist mit ihr ebenfalls ein massiver Fachkräfteabbau verbunden. Die im Beobachtungszeitraum erfolgte Reduzierung von minus 17,95 Personalstellen in Förderungsmaßnahmen (minus 58\%) ist mit einem Anstieg von $+25,91$ VZÄ an, sonstigem Personal (+76\%) ausgeglichen worden, bzw. um über 18\% angestiegen. Fördermaßnahmen waren 2018 meist ausgelaufen.

Ein Anstieg des sonstigen Personals kann z.B. eine Festeinstellung von Personal aus einer ehemaligen Fördermaßnahme, aber auch die Einstellung von neuen Berufsgruppen sein. Eine Fachqualifikation kann daher in dieser Personalgruppe nicht in Zahlen belegt werden.

${ }^{65}$ Naumann/Umlauf, S. 9. 
Aktuelle Situation der Öffentlichen Bibliotheken

in Sachsen-Anhalt

Tab.: 2 Personal an Öffentlichen Bibliotheken in Sachsen-Anhalt - laut DBS in VZÄ

\begin{tabular}{|c|c|c|c|c|c|c|c|}
\hline & $\begin{array}{c}\text { Personal } \\
\text { Fach- } \\
\text { bibliothekare }\end{array}$ & $\begin{array}{c}\text { Personal } \\
\text { Fach- } \\
\text { angestellte }\end{array}$ & $\begin{array}{c}\text { Summe } \\
\text { Fach- } \\
\text { personal }\end{array}$ & $\begin{array}{c}\text { Personal aus } \\
\text { Förder- } \\
\text { programmen }\end{array}$ & $\begin{array}{c}\text { Sonstiges } \\
\text { Personal }\end{array}$ & $\begin{array}{c}\text { Sonme } \\
\text { Sorsonaliges } \\
\text { Personal }\end{array}$ & $\begin{array}{c}\text { Personal } \\
\text { gesamt }\end{array}$ \\
\hline $\mathbf{2 0 0 7}$ & 152,77 & 109,47 & 262,24 & 30,98 & 34,25 & 65,23 & 327,47 \\
\hline $\mathbf{2 0 1 2}$ & 129,14 & 115,73 & 244,87 & 27,58 & 59,09 & 86,67 & 331,54 \\
\hline $\mathbf{2 0 1 7}$ & 117,52 & 109,79 & 227,31 & 13,03 & 60,16 & 73,19 & 300,5 \\
\hline & & & & & & & \\
\hline $\begin{array}{l}\text { Differenz } \\
\text { zu 2007 }\end{array}$ & $-35,25$ & 0,32 & $-34,93$ & $-17,95$ & 25,91 & 7,96 & $-26,97$ \\
\hline
\end{tabular}

Die Reduzierung des Gesamtpersonals um 27 VZÄ bei gleichbleibender Anzahl der Bibliotheken entspricht einem Wegfall der Personalbesetzung einer kompletten Großstadtbibliothek wie Ludwigsburg, Paderborn oder Regensburg.

Die Aufgaben des Bibliotheksfachpersonals (z.B. die Katalogisierung) werden von weniger Fachkräften geleistet, weshalb eine bessere Unterstützung bei diesen Tätigkeiten oder eine verbesserte kooperative Zusammenarbeit angebracht ist. 


\section{Einführung in die derzeitige Katalogisierungssituation}

In diesem Kapitel wird die bibliothekarische Katalogisierung in ihren Grundzügen umrissen. Dargestellt werden die allgemeinen Grundbegriffe und Vorgänge. Im Anschluss wird auf die Unterschiede in der Katalogisierung zwischen Wissenschaftlichen und Öffentlichen Bibliotheken eingegangen sowie die derzeitige Situation der Kooperationsverbünde dargestellt.

\subsection{Bibliothekarische Katalogisierung - Allgemeines}

Im bibliothekarischen Erschließungsvorgang werden Metadaten (Daten über Daten) erfasst. Diese Daten sind entweder im Dokument selbst enthalten oder sie werden von Bibliothekarinnen intellektuell erfasst oder maschinell erzeugt. ${ }^{66}$ Ziel ist es, eine Art Abbild des jeweiligen Mediums zu erstellen, mit dessen Hilfe der Bibliotheksnutzer das Medium im Katalog auffinden $\mathrm{kann}^{67}$. Der Arbeitsvorgang wird in Formal- und Sacherschließung unterteilt. „Die formale Erschließung setzt voraus, dass die Titeldaten erfasst, interpretiert und gegebenenfalls ergänzt werden. Die sachliche Erschließung setzt voraus, dass der Inhalt unabhängig von den Titeldaten analysiert und in geeignete Suchbegriffe (Schlagwörter oder Notationen) umgesetzt wird. ${ }^{68}$ Die bibliographische Beschreibung als Teil der Formalerschließung erfolgt nach den internationalen Standards der „International Standard Bibliographic Description“ (ISBD) ${ }^{69}$. Die in Deutschland eingesetzten Regelwerke für Alphabetische Katalogisierung haben die ISBD übernommen. Es finden sich jedoch kleinere Unterschiede, z.B. durch sprachliche Bezeichnungen oder Deskriptionszeichen. In den Öffentlichen Bibliotheken wird vorwiegend nach RAK-ÖB und jetzt auch nach RDA katalogisiert.

Die sachliche (inhaltliche) Erschließung wird durch die Vergabe von Schlagworten sowie durch die Vergabe einer Notation der Klassifikation der Bibliothek vorgenommen.

\subsection{Verwendete Standards}

Die einzelnen Inhalte in der bibliothekarischen Erschließung von Medien - formal und sachlich - werden in Datenfeldern erfasst. Diese Feldnummern sind durch das jeweilige Datenformat (z.B. internes Format, MAB2 oder MARC21) auf eine inhaltliche Zuordnung

\footnotetext{
${ }^{66}$ Haller/Fabian 2004, S. 223 ff.

${ }^{67}$ Wiesenmüller 2017, S. 30.

68 Ebenda, S. 224.

691971 erschien die ISBD(M) für monografische Publikationen. In der Folgezeit wurden für die unterschiedlichen Materialarten jeweils eigene ISBDs entwickelt, z. B. die ISBD(NBM) für Nichtbuchmaterialien und die ISBD(ER) für elektronische Ressourcen. 2011 wurden alle ISBDs in einem einzigen Text zusammengeführt („ISBD consolidated“) siehe: Wiesenmüller 2017, S.30.
} 
Einführung in die derzeitige Katalogisierungssituation

festgelegt. Die Anweisungen, wie das jeweilige Feld gefüllt wird, legt das verwendete Regelwerk (z.B. RAK-ÖB oder RDA) fest.

RDA - Resource Description and Access

Mit der Gründung des Standardisierungsausschusses $(\mathrm{STA})^{70} \mathrm{im}$ Jahr 2000 kam es zu Festlegungen, dass die deutschen Regelwerke und Formate eine internationale Neuausrichtung benötigen. 2011 wurde beschlossen, dass die deutschen Bibliotheken bis Ende 2015 auf RDA umsteigen.

Warum man das tun sollte, hat diverse Gründe. Der internationale Datenaustausch ist nur einer davon. Die Erfassung in Datenbanken und die Darstellung in Online-Katalogen bietet für Bibliotheken und deren Nutzer eine große Anzahl zusätzlicher Darstellungs- und Suchmöglichkeiten. So kann z.B. jede Person, die mit einem Werk in Beziehung steht, dargestellt werden - die Beschränkung in RAK kann entfallen, da nicht mehr für jede Person eine Katalogkarte gedruckt, in den Katalog eingestellt und wieder entfernt werden muss.

Die Darstellung in RDA ermöglicht eine detaillierte Auflistung jedes einzelnen Feldes und ermöglicht so eine semantische (maschinenlesbare) Darstellung. „Auf Basis von RDA erstellte Bibliothekskataloge werden einen maßgeblichen Beitrag zur Entwicklung des Semantic Web leisten, indem sie Begriffe, Benennungen und Bezeichnungen zusammenführen und Suchmaschinen erlauben, mehr zu leisten als Zeichenfolgen abzugleichen. " 71

RDA ist nach dem FRBR-Modell aufgebaut - in der Übersetzung: ,Funktionale Anforderungen an bibliographische Datensätze'.

Ziel der Einführung ist das Interesse des Nutzers, Dinge zu finden, sie zu identifizieren, auszuwählen und zu Ihnen Zugang zu erhalten. Die Umsetzung erfolgt in einem an relationale Datenbanken angelehnten Entitätenmodell. Die Entitäten werden in 3 Gruppen unterteilt und stehen jeweils in Beziehung zueinander:

Gruppe 1: Werk-Expression-Manifestation-Exemplar

Gruppe 2: Person-Körperschaft

Gruppe 3: Begriff-Gegenstand-Ereignis-Ort ${ }^{72}$

\footnotetext{
${ }^{70}$ Ein Zusammenschluss von wiss. Bibliotheken, Verbundsystemen, politischen Vertretungen...um im deutschsprachigen Raum einheitliche bibliothekarische Standards sicherzustellen. Siehe: Wiesenmüller 2017, S. 5.

${ }^{71}$ Umlauf 2015 http://www.ib.hu-berlin.de/\%7Ekumlau/handreichungen/h189/ (07.02.2019).

72 Vgl.: Wiesenmüller 2017, S. 11 - 30.
} 
Einführung in die derzeitige Katalogisierungssituation

\section{GND}

Für eine normierte Ansetzungsform von Personennamen, Schlagworten und z.B. Körperschaften steht seit 2012 die GND ${ }^{73}$ unter einer frei nachnutzbaren CC0 1.0 $0^{74}$-Lizenz zur Verfügung. Die Umfrage in Sachsen-Anhalt ergab, dass mit 18\% 7 der teilnehmenden Bibliotheken die GND nutzen. Die 3 Großstadtbibliotheken sind dabei nicht vertreten. Die hier jeweils eingesetzte Bibliotheksmanagementsoftware BIBLIOTHECAplus bietet keinen SRU-Abgleich und somit könnte die GND nur regelmäßig komplett in eine 2. Datenbank importiert werden. (siehe Fazit)

\section{Austauschformate}

Die bibliographische Beschreibung wird in einer konkreten Datenbankstruktur abgebildet. Dieses als Datenformat bezeichnete Schema legt fest, in welches Datenbankfeld ein typologisch gleicher Inhalt abgelegt wird. Um Metadaten untereinander austauschen zu können, bedarf es neben vorhandener Datenstrukturen auch Übertragungsprotokolle und Datenaustauschformate. Bibliotheksmanagementsysteme verwenden für die Speicherung von Katalogisierungsdaten interne Datenformate. Der Import und Export der Daten wird gewährleistet, indem diese Formate in standardisierte Austauschformate konvertiert werden. ${ }^{75}$ Seit 1973 liegt in Deutschland das "Maschinelle Austauschformat für Bibliotheken (MAB)“ als einheitliches Datenformat für den Austausch bibliografischer Daten in maschinenlesbarer Form vor. Die Weiterentwicklung MAB2 ermöglicht es den deutschen Bibliotheken seit 1995 auch, Daten über Online-Umgebungen auszutauschen. ${ }^{76} 2006$ wurde die Weiterentwicklung dieses Formates eingestellt, 2013 endete auch die Datenlieferung der DNB im Format MAB2. ${ }^{77}$

MAB2 ist ein in den Öffentlichen Bibliotheken noch sehr häufig genutztes Datenformat. Nicht alle Bibliotheksmanagementsysteme ermöglichen einen vollständigen automatisierten Fremddatenaustausch über MARC21 in RDA. (siehe Anhang 3)

In Deutschland wird seit 2013 versucht, das Format MAchine-Readable Cataloging (MARC21) durchzusetzen. MARC21 ist ein von der Library of Congress entwickeltes Datenformat, das verschiedene Formen von Metadaten wie Titeldaten, Normdaten und Exemplardaten, Klassifikationen und Adressdaten abbilden kann. MARC21 kann in der offenen Auszeichnungssprache XML als MARCXML ausgedrückt werden.

\footnotetext{
73 Siehe: Deutsche Nationalbibliothek 2019a. https://wiki.dnb.de/display/ILTIS/Informationsseite+zur+GND (25.02.2019).

${ }^{74} \mathrm{vgl}$. https://creativecommons.org/publicdomain/zero/1.0/deed.de (25.02.2019).

75 Plassmann et al. 2011, S. 206.

76 Umlauf 2016, S. 205.

${ }^{77}$ https://www.dnb.de/DE/Standardisierung/Formate/MAB/mab_node.html (07.02.2019).
} 
In Kombination mit dem Regelwerk RDA kann ein Austausch und eine Nachnutzung von Metadaten plattform- und herstellerunabhängig realisiert werden. ${ }^{78} \mathrm{In}$ den Experteninterviews der Systemanbieter der vier eingesetzten Bibliotheksmanagementsysteme wurde deutlich, dass diese Systeme MARC21-Daten importieren können. 46\% der befragten Bibliotheken (18) setzen jedoch noch MAB2 für ihren Datenimport ein. 12 Bibliotheken (31\%) gaben ,andere' Formate an, wobei eine mangelnde Kenntnis der Datenformate deutlich wird. Alle vier eingesetzten Bibliothekssysteme nutzen für das Ablegen der Daten ein internes Datenformat. Um Daten importieren oder exportieren zu können, werden die Daten in MAB2 oder MARC21 konvertiert.

Jedoch können nicht alle Vorteile, die RDA bietet, in MARC21 angezeigt werden. Seit 2011 entwickelt die BIBFRAME ${ }^{79}$-Initiative der Library of Congress ein neues Framework, das insbesondere die semantische Auszeichnung der bibliographischen Daten in den Vordergrund rückt. BIBFRAME soll MARC21 mittelfristig ablösen. ${ }^{80}$ Die DNB stellt als Mitglied der „Early Implementers Group" seit Februar 2014 eine prototypische Konversion über einen Link in den Titelanzeigen des DNB-Kataloges zur Verfügung. ${ }^{81}$

Die 4 Anbieter der in Sachsen-Anhalts Öffentlichen Bibliotheken eingesetzten Bibliotheksmanagementsoftware setzen sich zurzeit noch nicht mit BIBFRAME auseinander.

\section{Übertragungsprotokolle}

Das Datenübertragungsprotokoll Z39.50 ist ein standardisiertes Kommunikationsprotokoll für den Datenaustausch. Das Protokoll wurde von der National Standards Organisation (NISO) seit 1984 entwickelt und wird seit 1990 durch die Library of Congress weiterbetreut. ${ }^{82}$ Es „fungiert als universeller Übersetzer zwischen dem Zugriffsprogramm und dem Datenbanksystem. ${ }^{183}$ In einer Client-Server-Anwendung werden die Daten verbindungsorientiert übertragen. Das Protokoll ermöglicht sowohl die Suche als auch die Abruf-Funktion und ist in der Lage parallel mehrere Datenbanken abzufragen. Die im Projekt „DBV-OSI84${ }^{84}$ standardisiertes WebserviceProtokoll“ festgelegten Standards wurden von den deutschen Verbünden aufgegriffen und es konnte somit schon Ende der 1990er Jahre eine Datenübertragung erfolgen.

\footnotetext{
78 Schade 2016, S. 71.

${ }^{79}$ Bibliographic Framework Initiative (BIBFRAME). o.J. http://bibframe.org (07.02.2019).

${ }^{80}$ Gantert 2016, S. 221.

81 https://www.dnb.de/DE/Wir/Projekte/Laufend/bibframe.html (07.02.2019).

82 Göttker/Wein 2014, S. 76.

${ }^{83}$ Plassmann et al. 2011, S. 206.

${ }^{84}$ DBV-OSI Projekt (Deutscher Bibliothekenverbund - Open Systems Interconnection) ein vom Bundesministerium für Bildung, Wissenschaft, Forschung und Technologie und der Deutschen Forschungsgemeinschaft (DFG) gefördertes Projekt (1993 - 1997) Entwicklung eines z39.50 server und z39.50 client.
} 
Die Verbindung findet in einer systemunabhängigen Transfer-Syntax statt. Basis ist das Bib1Attribut Set ${ }^{85}$ der Library of Congress. Z39.50 wird vorwiegend im Bibliotheksumfeld eingesetzt und ist insbesondere im Bereich der Fernleihe auch heute noch ein in den Verbünden genutztes Protokoll. Durch die Integration von Z39.50 Clients in die Bibliotheksmanagementsysteme wurde ein Abfragen von Metadaten auch den Öffentlichen Bibliotheken möglich. Z39.50 ist jedoch kein Protokoll der Internet-Zeit. Durch Gateways wie den ZACK wurde zwar eine Übertragung über das Internet möglich gemacht, die Entwicklung ist jedoch seit dem Jahr 2000 in Richtung HTTP-bzw. HTTPS-Protokoll weitergegangen. Deren Vorteil liegt in Verwendung neuer Protokolle oder Frameworks, die in allgemein genutzten Formaten webbasiert übertragen werden. Mit einer Nutzung wäre eine Basis gelegt, dass sie auch von Systemen und Programmierern außerhalb des Bibliothekswesens verstanden und bearbeitet werden können. ${ }^{86}$

Eine Weiterentwicklung von Z39.50 ist das standardisierte Webservice-Protokoll SRU (Search / Retrieve via URL) ${ }^{87}$. Auch dieser Standard wurde von der Library of Congress entwickelt. Die Anfragen werden in der Retrievalsprache CQL (Contextual Query Language) formuliert. Ziel ist die URL-basierte Abfrage von Datenbanken über das HTTP oder HTTPS-Protokoll. Bei der beibehaltenen Suchsemantik von Z39.50 wird die Abfrage hiermit zustandslos, also ohne Session durchgeführt. Somit wird hier ein grundlegender Architekturwandel - vom Client/Server zum Webbasierten System vollzogen. Die Ergebnisse der Suche werden in einem XML-Format zur Verfügung gestellt. Über eine gezielte Suche mittels Suchindizes und Suchbegriffen ermöglicht das Protokoll die Übernahme der Ergebnisse in die eigene Umgebung. ${ }^{88}$

Durch die Abfrage über HTTPS entfällt für die Bibliotheken die Freischaltung der konkreten Ports der Z39.50 Server der Datenbankanwender, die sich zunehmend als Unsicherheitsfaktor in der Absicherung des Webservers und als Problem bei der Konfiguration der Firewall erweisen.

Neben dem Übertragungsprotokoll Z39.50 und dem XML-basierten Übertragungsprotokoll SRU wird der Einsatz von offenen Übertragungsprotokollen notwendig. ${ }^{89}$ Diese Entwicklung kann dem Gesamtsystem der Bibliotheken weltweit den Vorteil bringen, dass Bibliotheks-IT keine Nischenanwendung mehr sein wird.

\footnotetext{
85 Die Feldbezeichnungen des BIB1-Attributs siehe: https://www.loc.gov/z3950/agency/bib1.html (09.03.2019).

${ }^{86}$ Siehe: Pohl/Danowski 2013, S. 2.

87 Siehe: Library of Congress 2016 (25.02.2019).

${ }^{88}$ Deutsche Nationalbibliothek 2018a. (25.02.2019).

${ }^{89}$ Schade 2016, S. 71.
} 


\subsection{Unterscheidung der Katalogisierungsarbeit in Wissenschaftlichen und Öffentlichen Bibliotheken}

Der Vorgang der Katalogisierung unterscheidet sich in einer Verbundlösung wesentlich von einer Katalogisierung ausschließlich für die eigene Bibliothek. Die Verbundzentralen bieten den Mitgliedsbibliotheken die Möglichkeit einer Online-Katalogisierung auf der Grundlage einer existierenden Datenbank. Sie bieten einen stets aktuellen Zugang zur GND sowie zu weiteren Metadaten, Datenbanken und E-Books und legen wegen der heterogenen Formate Verbundrichtlinien fest und schulen diese. ${ }^{90}$

Die Arbeit der Verbundsysteme wird seit 1983 durch die ,Arbeitsgemeinschaft der Verbundsysteme (AGV)' begleitet, deren Ziel die verbundübergreifende Koordinierung von Konzepten und die Kooperation bei der Einführung und dem Betrieb von innovativen Dienstleistungen ist. Als eine von zwei ständigen Arbeitsgruppen erstellt die AG Leihverkehr Empfehlungen für einen deutschlandweit optimal umgesetzten Leihverkehr und die AG Kooperative Verbundanwendungen erarbeitet Maßnahmen zur Verbesserung der Datenübernahme zwischen den Verbünden mit dem Ziel der Senkung der Eigenkatalogisierungsquote sowie die Anreicherung von Katalogisaten mit zusätzlichen Informationen. ${ }^{91}$

Verbundbibliotheken vollziehen ihre Katalogisierung nach vorgegebenen Bearbeitungsrichtlinien der Verbundzentrale..$^{92}$ Oberste Priorität hat dabei die Kontrolle, ob das vorliegende Medium schon erfasst ist. Je nach Vorhanden- oder Nichtvorhandensein, wird das Katalogisat angelegt (wenn die Rechte vorhanden sind) oder das Exemplar wird angehängt. Die Unterschiede zeichnen sich z.B. durch die Verwendung der Normdatensätze ab. Die Katalogisiererin in einem Verbund verknüpft und aktualisiert die vorhandenen Einträge. In der Öffentlichen Bibliothek liegen z.B. die Personendatensätze komplett mit in der Titelaufnahme in der lokalen Datenbank. Sie werden mit den eigenen Einträgen verknüpft, oder - wenn möglich - mit den GND-Daten abgeglichen. Die Titelaufnahmen werden jedoch zu einem hohen Anteil manuell erfasst. Durch das Einspielen der Datensätze in den ÖVK ist auch bei den Öffentlichen Bibliotheken ein Vorgehen streng nach vorliegenden Katalogisierungsregeln angemessen, da die Daten sichtbar im ÖVK dargestellt werden. Die Unterschiede in den individuellen Katalogisierungsvorgaben der einzelnen Bibliotheken führen zurzeit jedoch noch zu häufigen Mehrfachausgaben der aufgenommenen Titel.

\footnotetext{
90 Hohoff 2015, S. 83.

${ }^{91}$ Siehe: https://www.dnb.de/DE/Wir/Kooperation/AGVerbundsysteme/agverbund_node.html.(19.02.2019).

92 Siehe z.B. unter: Verbundzentrale des GBV o.J.a. Richtlinie zur Bearbeitung von Titelaufnahmen im GBVKat mit Anhängen.
} 


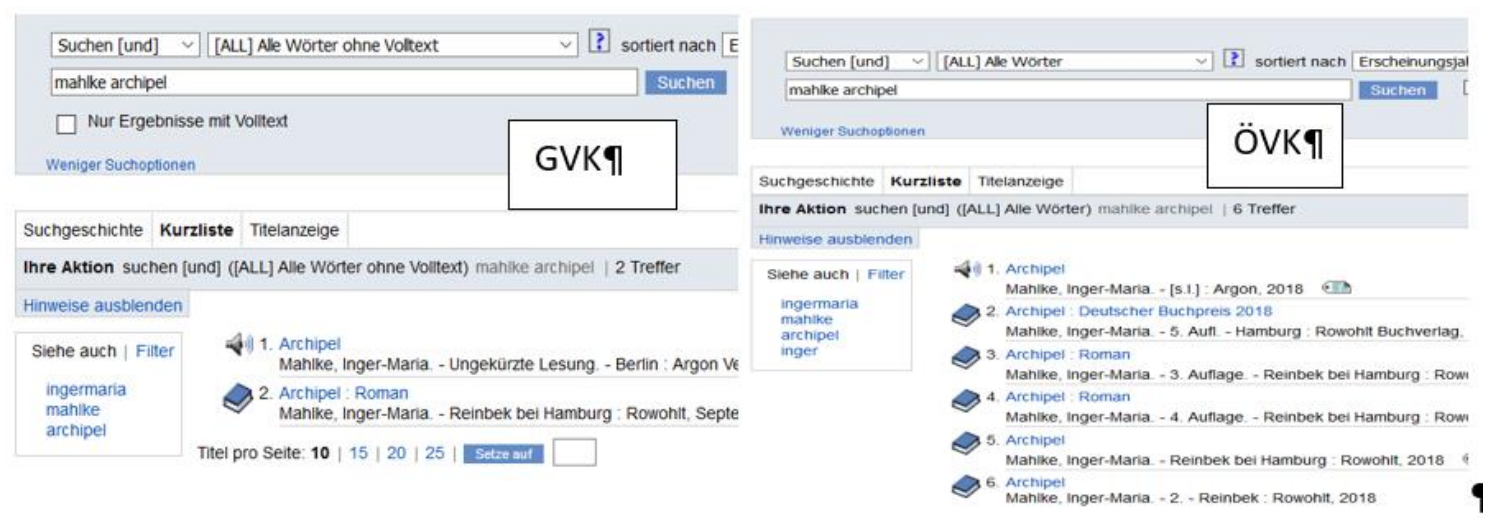

Abb.: 3 Darstellung einer identischen Suche in GVK und ÖVK

Die Beispiele zeigen die Unterschiede in den Trefferanzeigen zwischen GVK und ÖVK bei der identischen Suche nach ,mahlke archipel‘. Im GVK werden die Exemplare der Bibliotheken an eine erste identische Titelaufnahme angehängt. Es sind RDA-Aufnahmen - daher besteht auch keine unterschiedliche Anzeige der verschiedenen unveränderten Nachauflagen des Romans. Alle Exemplare des Werkes ,Archipel' können in einer Titelaufnahme dargestellt und somit gesucht werden. Anders bei den ÖVK-Titelaufnahmen: Hier zeigen die unterschiedlichen Ansetzungen z.B. des Verlagsortes Reinbek bei Hamburg, die Unterschiede im Ausschreiben des Wortes ,Auflage‘ und die unterschiedlichen Zusätze zum Sachtitel deutlich die verschiedenen Handschriften der Kolleginnen sowie die unterschiedlichen Katalogisierungsregeln. Die Datenzusammenführung des ÖVK hin zum ,Master-Record-Model' sollte hier eine deutliche Verbesserung bringen. 


\subsection{Verbünde}

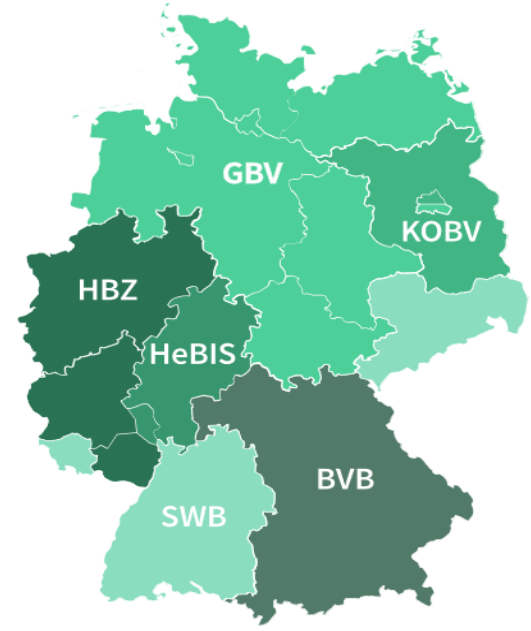

Abb.: 4 Bibliotheksverbünde in Deutschland - nach Regionen ${ }^{93}$

Wissenschaftliche Bibliotheken haben sich seit den 1970er Jahren zu regionalen Bibliotheksverbünden zusammengeschlossen. Vorrangiges Ziel war es, die einmal katalogisierten Daten gegenseitig nutzbar zu machen. Die entstandenen Datenbanken ermöglichen eine überregionale Suche und wurden somit zu wichtigen Instrumenten für Recherchen und die Steuerung des deutschen Leihverkehrs ${ }^{94}$. Entstanden ist in Deutschland jedoch nicht eine deutschlandweite Datenbank, sondern es existieren derzeit 5 Verbunddatenbanken, verteilt auf sechs Bibliotheksverbünde ${ }^{95}$.

Von Seiten der Deutschen Forschungsgemeinschaft (DFG) und dem Wissenschaftsrat (WR) wurden in den Jahren 2010/2011 nachhaltige Konsolidierungsprozesse und neue Strukturreformen gefordert. ${ }^{96}$ In einer ,gemeinsamen Erklärung ${ }^{97}$ und einem Positionspapier ${ }^{98}$ wurden diese aufgelistet und formuliert.

Als Konsequenz der auf die beiden Papiere folgenden Ausschreibung der DFG wurde 2013 das CIB-Projekt (Cloudbasierte Infrastruktur für Bibliotheksdaten) ${ }^{99}$ gestartet. Projektpartner waren das Hessische BibliotheksInformationsSystem (HeBIS) als Konsortialführer, der Bibliotheksverbund Bayern (BVB) und der Kooperative Bibliotheksverbund Berlin-Brandenburg

\footnotetext{
93 Quelle: Deutscher Bibliotheksverband: Bibliotheksportal/ Verbundsysteme in Deutschland. https://bibliotheksportal.de/informationen/bibliothekslandschaft/bibliotheksverbuende/ (12.01.2019).

94 Plassmann et al. 2011, S. 154.

95 Die Verteilung der Bundesländer auf Bibliotheksverbünde ist in Abbildung 4 dargestellt.

96 Diedrichs/Goebel 2018, S. 211.

97 Deutsche Forschungsgemeinschaft und Wissenschaftsrat 2011a. 2011.

98 Deutsche Forschungsgemeinschaft und Wissenschaftsrat 2011b.

${ }^{99}$ Siehe auch: http://www.projekt-cib.de/wordpress/ (14.02.2019).
} 
Einführung in die derzeitige Katalogisierungssituation

$(\mathrm{KOBV})^{100}$. Das Projekt konnte Wege in eine cloudbasierte und international ausgerichtete Infrastruktur untersuchen und wesentliche Grundbedingungen schaffen.

Als Resümee des CIB-Projektes wurde festgehalten: „Die Schaffung eines einheitlichen deutschen Datenraumes mithilfe der Plattformsynchronisation konnte nicht gelingen." 101 Empfohlen wurde vom 2016 abgeschlossenen Projekt eine Primärkatalogisierung im OCLC WorldCat ${ }^{102}$.

Im Ergebnis der Expertenbefragung wird deutlich, dass OCLC für die Öffentlichen Bibliotheken mit der Bibliotheksmanagementsoftware BIBLIOTHECAplus auch den WorldCat im Fokus hat.

Während des CIB-Projektzeitraumes einigten sich das BSZ und der GBV auf eine strategische Partnerschaft ihrer beiden Verbünde. ${ }^{103}$ Die Datenbank ,K10plus' enthält die Daten von Bibliotheken aus 10 Bundesländern sowie der Stiftung Preußischer Kulturbesitz. Für den 25. März 2019 ist der Produktionsbeginn geplant. ${ }^{104}$ Das Index-Backend ,K10plus-Zentral' wird mit mehr als 200 Millionen Datensätzen neben dem GVK, dem SWB und vielen weiteren Daten auch den ÖVK enthalten.

Das Angebot des GBV für Wissenschaftliche Bibliotheken ist sehr komplex. Zum Abgabezeitpunkt dieser Arbeit wird sich die Katalogisierung der Bibliotheken gerade in der Umstellungsphase befinden.

Ein Kooperationsprojekt aller Verbünde stellt der ,Gemeinsame Verbünde-Index bzw. FernleihIndex ${ }^{105}$ der AG Verbundsysteme dar. In einer Solr-Cloud werden zurzeit die Index-Daten von ca. 170 Mio. Titeln vorgehalten und täglich aktualisiert. Über das Open Source Discovery-System VuFind wurde das Fernleihportal ,BOSS ${ }^{106}$ aufgesetzt. BOSS bietet über eine einfach zu bedienende Oberfläche und über eine Facettierung den schnellen Zugriff auf das gewünschte Medium, das dann über eine Fernleihbestellung oder einen Online-Zugriff genutzt werden kann. ${ }^{107}$ Als externer Index kann er in bestehende Portale eingebunden werden. (Bsp. KOBV ${ }^{108}$ ) Die folgende Tabelle stellt die bestehenden und geplanten Kooperationen der Bibliotheksverbünde in einer Übersicht dar.

\footnotetext{
100 Röschlau 2017, S. 411.

101 Kratzer et al. 2016, S. 254

102 https://www.worldcat.org/ (14.02.2019).

103 Diedrichs/Goebel 2018, S. 213.

104 https://wiki.k10plus.de/display/STAR/Startseite (14.02.2019).

105 Siehe: https://wiki.bsz-bw.de/doku.php?id=projekte:boss:start (11.03.2019).

106 Zu nutzen über: https://fernleihe.boss.bsz-bw.de/ (11.03.2019).

107 Vgl. Conradt/Lohrum 2017.

108 Zu nutzen über: https://portal.kobv.de/?plv=2 (11.03.2019).
} 
Tab.: 3 Verbunddatenbanken in Deutschland: ${ }^{109}$

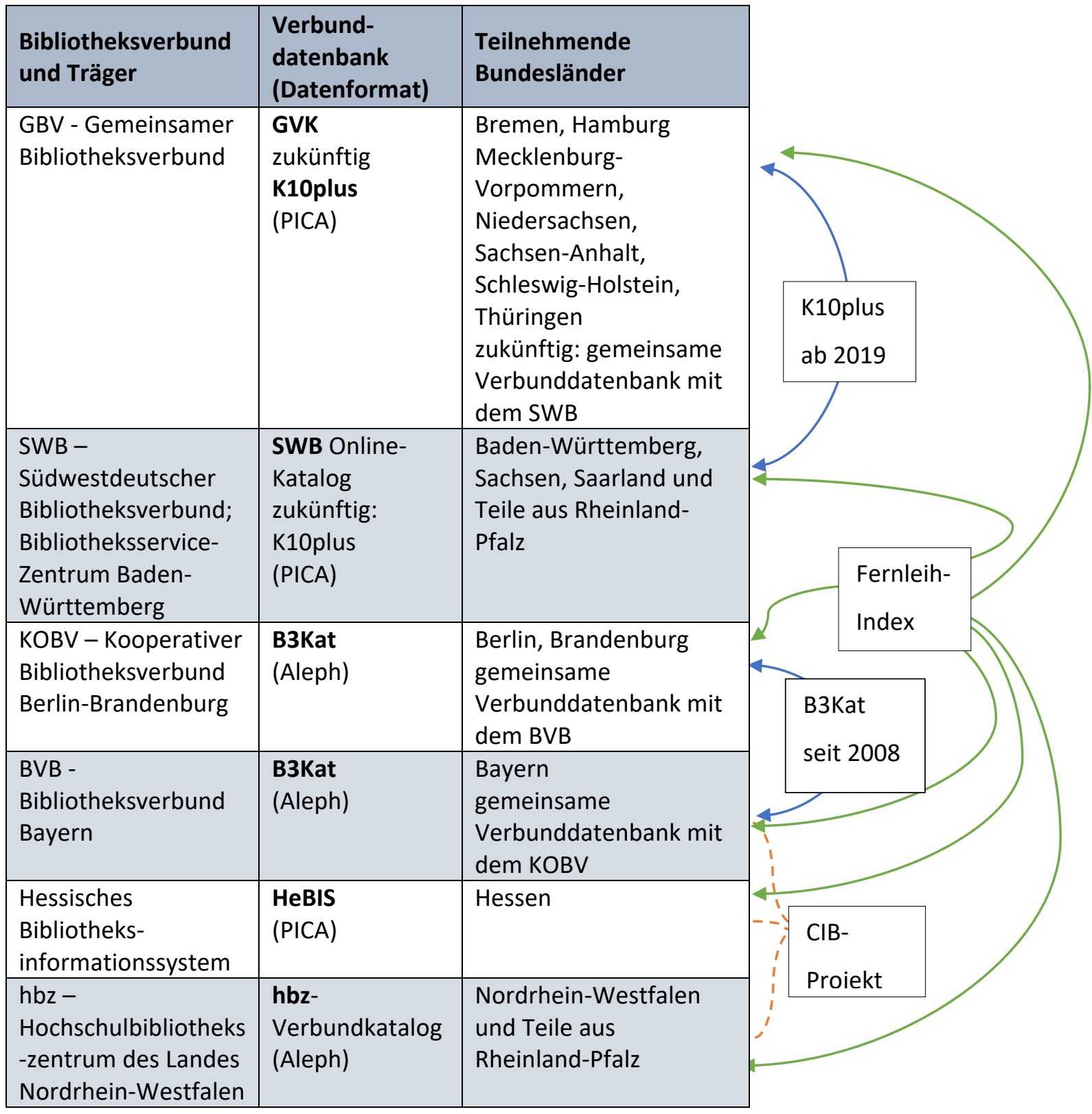

Der GBV bietet den Öffentlichen Bibliotheken der am Verbund teilnehmenden Bundesländer seit 2004 eine separate Datenbank an - den ÖVK. (siehe 2.2) 


\section{Umfrage zur Katalogisierungssituation in Öffentlichen Bibliotheken Sachsen-Anhalts}

In diesem Kapitel wird das Kernstück der Arbeit - die Umfrage zur Katalogisierungssituation erläutert und ausgewertet. Zunächst wird die Methodik der Durchführung vorgestellt, hierzu wird die Grundlage der Daten, die Umfrageart und -durchführung sowie die Fragenzusammenstellung genauer beschrieben. Im Anschluss erfolgt die Auswertung der Fragenkomplexe in dem jeweiligen inhaltlichen Antwortkontext.

\subsection{Methodik}

Um die derzeitige Katalogisierungssituation und den Stand der Fremddatenübernahme in den Öffentlichen Bibliotheken Sachsen-Anhalts eruieren zu können, wurde eine Online-Umfrage konzipiert. Diese Lösung wurde gewählt, um auf einfachem Weg möglichst viele Bibliotheken des Bundeslandes zu erreichen. Zur Abgrenzung der Zielgruppe wurde zunächst eine Auflistung der Landesfachstelle für Öffentliche Bibliotheken des Landes Sachsen-Anhalt mit 75 hauptamtlich geführten Bibliotheken ${ }^{110}$ verwendet. Abgeglichen wurde diese Liste mit der DBSStatistik ${ }^{111}$ für 2017, die 74 Bibliotheken in der Auswahl: 'Öffentliche Bibliothek, Sachsen-Anhalt, hauptamtlich geführt' ausweist. Die 75 Bibliotheken wurden per E-Mail kontaktiert und bilden somit die Grundgesamtheit.

Aufgrund der kleinen Grundgesamtheit und der durch die im Masterarbeitsthema räumlich und inhaltlich schon vorgegebenen Einschränkungen kam nur eine Vollerhebung in Frage. Die Komplexität des Themas und die zu erwartende Individualität der Antworten führten zu einer kombinierten Forschungsmethode aus quantitativem und qualitativem Design. Diese Methode ermöglicht es, sowohl die Rahmenbedingungen als auch die persönlichen Einschätzungen abzufragen. Die Umfrage unterteilt die Teilnehmenden in der ersten Frage in Bibliotheksleitung und Mitarbeiterinnen in der Katalogisierung. Katalogisiererinnen wurden nach Auswahl ihrer Tätigkeit per Filter gleich auf diejenigen Fragen geleitet, die nur Katalogisierung und Fremddatenübernahme betreffen. (ab Frage 19ff)

Die Umfrage bestand aus 24 Fragen und 2 Zusatzfragen. Es wurden offene, halboffene, geschlossene Fragen und Bewertungsfragen eingesetzt. Da sich die Umfrage auf einen relativ kleinen geschlossenen Teilnehmerkreis bezog, wurden sämtliche Fragen als Pflichtfragen festgelegt. Es wurden jedoch Möglichkeiten der Nichtbeantwortung durch Felder wie, nicht

\footnotetext{
110 https://Ivwa.sachsen-anhalt.de/das-Ivwa/kultur-denkmalschutz/landesfachstelle-fuer-oeffentlichebibliotheken/bibliotheken-in-sachsen-anhalt/ (15.01.2019).

${ }^{111}$ Deutsche Bibliotheksstatistik siehe: https://www.bibliotheksstatistik.de/ (15.01.2019).
} 
Umfrage zur Katalogisierungssituation in Öffentlichen Bibliotheken Sachsen-Anhalts

bekannt' oder ,Sonstiges' eingeräumt. Nach Möglichkeit wurden geschlossene Fragen mit vorgegebenen Antwortmöglichkeiten vorgelegt, um die Beantwortung möglichst einfach zu halten. Den Fragen wurde in einigen Fällen ein erläuternder Hilfstext beigefügt. Auf die Möglichkeit von Mehrfachantworten wurde hingewiesen. Mit Hilfe von einstellbaren Logiken und Filtern wurden bei Beantwortung bestimmter Fragen je nach ausgewählter Antwort die sich darauf beziehenden Folgefragen angezeigt oder übersprungen. So wurden die Katalogisiererinnen bei Beantwortung der Frage 1 sofort auf Frage 19 weitergeleitet. Die Fragen $13^{112}$ und Frage $14^{113}$ wurde nur bei Verneinung der Frage $12^{114}$ angezeigt. Ein Filter bei Frage $19^{115}$ führte bei Markierung der Antwort, Ich nutze keine Fremddaten' zur Frage $\mathbf{2 1}^{116}$. (siehe Abbildung 5)

Der Fragenkatalog wurde in 3 inhaltliche Bereiche grob unterteilt:

1. Grundsatzfragen / Rahmenbedingungen: u. a. Einwohnerzahl, Mitarbeiteranzahl und eingesetztes Bibliotheksmanagementsystem (Fragen 1-4),

2. technische Voraussetzungen in der Bibliothek, verwendete Datenformate/ Schnittstellen,

Teilnahme am ÖVK (Fragen 5-14),

3. inhaltliche Aspekte von Katalogisierung und Fremddatenübernahmen (Bibliothek: Fragen 15-18; individuell: Fragen 19-24).

Die Aussagen der 1. Fragengruppe stellten sicher, dass es sich bei den Antworten um unterschiedliche Bibliotheken handelt. Ein zusätzliches Statistikmerkmal verwies auf den jeweiligen Landkreis. Dies ermöglichte es, die Antworten weitgehend auf Plausibilität zu überprüfen.

Die Fragengruppen der Bereiche 1, 2 und 3 (bis Frage 18) sollten von der jeweiligen Bibliotheksleitung beantwortet werden. Die Fragen zum 3. Themenbereich (ab Frage 19) sind den Katalogisiererinnen vorbehalten. Da in einer Reihe von Bibliotheken die Leitung zusätzlich Katalogisierungsaufgaben übernimmt (u.a. in One-Person-Libraries), konnte die Beantwortung ab Frage 19 auch von der Bibliotheksleitung weiter ausgefüllt werden. Ist dies nicht der Fall, gab

\footnotetext{
112 Frage 13: ,Warum nehmen Sie nicht am ÖVK teil?’

113 Frage 14: ,Wenn eine laufende - möglichst automatisierte - Aktualisierung der Katalogdaten dazu führen würde, dass Sie aktuelle Titeldaten direkt aus dem ÖVK importieren könnten, würde Ihre Bibliothek dann dem ÖVK beitreten?"

114 Frage 12: „Nimmt Ihre Bibliothek am ÖVK teil?’

115 Frage 19: ,Welche Anbieter von Katalogdaten bzw. welche Werkzeuge nutzen Sie PERSÖNLICH zur manuellen Katalogisierung, indem Sie Daten kopieren und in Ihren Katalog einfügen?’

${ }^{116}$ Frage 21: ,Warum nutzen Sie persönlich keine Fremddaten?’
} 
Umfrage zur Katalogisierungssituation in Öffentlichen Bibliotheken Sachsen-Anhalts

es eine verneinende Antwortmöglichkeit bei den Katalogisierungsfragen. Dieses Vorgehen erschien wegen der individuellen Vorgehensweise einer jeden Katalogisiererin am meisten zielführend. Basiert die Katalogisierung nicht auf einer automatisierten Übernahmemöglichkeit von Metadaten, erfordert der Arbeitsprozess einen hohen Eigenanteil an Recherchearbeit und individuellen Suchwegen. Diese Mitarbeiterinnengruppe wurde daher separat angesprochen. Die Bibliotheksleitung war aufgefordert, den Umfragelink an Personal in der Katalogisierung weiterzuleiten.

In Frage 25 wurde abgefragt, wer Interesse an einem generellen Austausch über das Thema Katalogisierung hat und in Frage $\mathbf{2 6}$ wurde mit der Gewinnaussicht auf kleine Bürohilfen ein Anreiz gesetzt, an der Umfrage teilzunehmen.

Technisch wurde die Online-Umfrage mit dem Tool ,easyfeedback ${ }^{117}$ umgesetzt, das in einem modernen Webdesign datenschutzkonform alle notwendigen Features anbietet.

Nach der Erstellung der Fragen wurde ein Pretest mit 6 Testpersonen aus verschiedenen Bibliotheken bzw. Institutionen durchgeführt. Die nun angepassten Fragen wurden ab dem 27.11.2018 per E-Mail-Verteiler an die Bibliotheksleitungen geschickt. Um den Zugriff möglichst unproblematisch zu gestalten, wurde auf das Erstellen von persönlichen Zugangscodes verzichtet, da sich dieses Verfahren anhand des Pretests für eine solche kombinierte Umfrage als nicht handhabbar herausstellte. Nach 2 Wochen wurde eine Erinnerung von Seiten der Landesfachstelle geschickt, in der um Teilnahme an der Umfrage gebeten wurde.

${ }^{117}$ Easyfeedback siehe: https://easy-feedback.de/ (15.01.2019). 


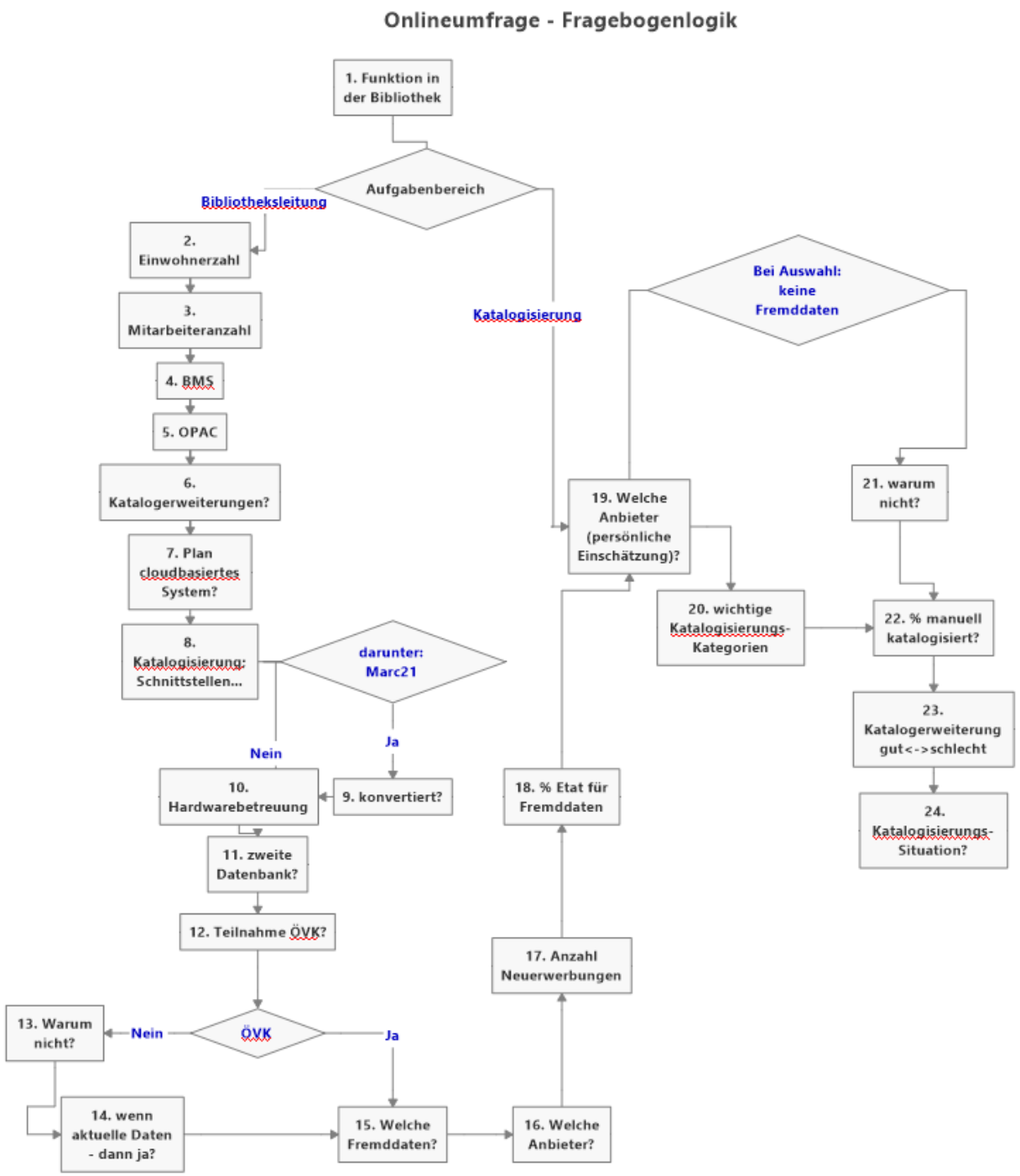

Abb.: 5 Darstellung von Logik und Filter in der Fragestellung der Umfrage - eigene Darstellung

\subsection{Auswertung der Umfrage}

\subsubsection{Teilnahme}

Das Umfragetool registrierte 71 Teilnehmende, von denen 59 die Umfrage vollständig und 12 teilweise beendet haben. Aus der Antwortgruppe der unvollständigen Beantwortungen wurden alle Teilnehmende mit mehr als 4 beantworteten Fragen (7) in die Analyse einbezogen und als auswertbar angesehen. Antworten mit weniger als 5 beantworteten Fragen (5) wurden gelöscht, da es sich hier durch Festlegung auf Pflichtfragen nur um die Grundsatzfragen handelt und somit kein inhaltlicher Bezug zur Thematik der Arbeit hergestellt werden kann. Somit können 93\% (66 Teilnehmende) in die Analyse einbezogen werden. 
Die Fragen 1 bis 4 dienen der Zuordnung der Antwortenden zu einer Tätigkeitsgruppe in der Bibliothek, der eingesetzten Bibliotheksmanagementsoftware, sowie der Gemeinde- und Personalgröße. Ab Frage 5 beginnt die inhaltliche Zuordnung zum analysierten Thema. Nicht feststellbar ist, ob weitere Bibliotheken die Umfrage begonnen haben oder ob Teilnehmende vorab getestet, also die Umfrage abgebrochen und später erneut begonnen haben. Anhand der Antworten wurden keine Dopplungen festgestellt. Ebenfalls stimmen die Antworten mit der jeweiligen Zuordnung zum Landkreis überein. ${ }^{118}$

Geantwortet haben 47 Bibliotheken, was einer Gesamtbeteiligung von 63 \% entspricht. Fünf Bibliotheken - bis 30.000 zu betreuende Einwohner - haben die Umfrage abgebrochen, bevor eine inhaltliche Beantwortung erfolgt ist. Die Datenbasis wurde daraufhin um diese Teilnehmende bereinigt. Sieben Bibliotheken haben nicht alle Fragen ausgefüllt - hier werden die Antworten einbezogen, die auswertbar sind. Eine Bibliothek hat nur Fragen des ThemenBereichs ,Katalogisierung' beantwortet. Eine Auswertung ist daher bei $n=42$ Bibliotheken möglich. Bei manchen Fragen liegt der Grundausgangswert aus den genannten Gründen niedriger und wird somit in den Berechnungen jeweils angepasst. Von den 26 Bibliotheken in Gemeinden mit mehr als 20.000 zu betreuenden Einwohnern haben mit 22 teilnehmenden Bibliotheken 85 \% der Bibliotheken geantwortet. Diese Umfrage stellt daher die derzeitige Katalogisierungs-Situation der hauptamtlich geführten Öffentlichen Bibliotheken in SachsenAnhalt deutlich dar.

Als ,Mitarbeiter der Katalogisierung' haben 25 Teilnehmende geantwortet. Diese Antworten werden parallel zu den institutionellen Beantwortungen ausgewertet.

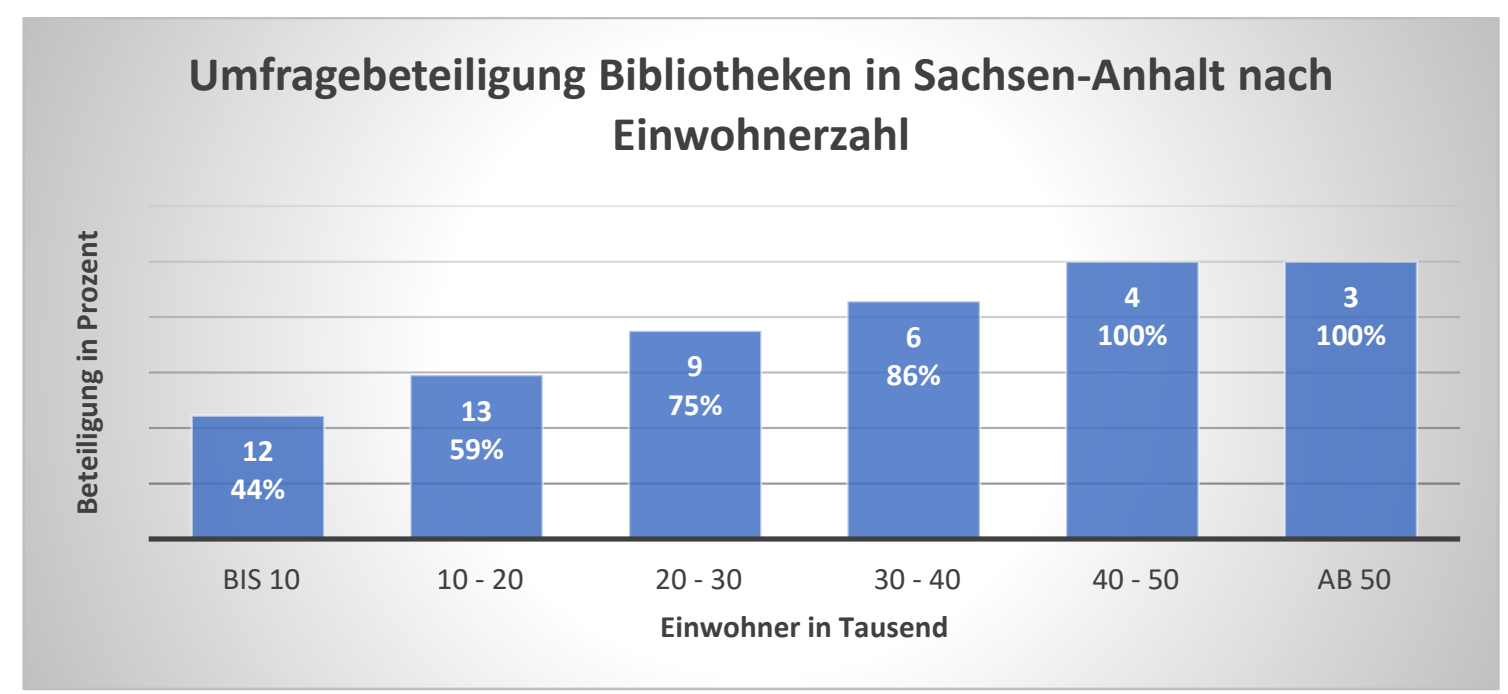

Abb.: 6 Umfragebeteiligung der Öffentlichen Bibliotheken nach Einwohnerzahl der Gemeinden (Anzahl Bibliotheken; prozentualer Anteil) ( $n=47)$

${ }^{118}$ Bsp.: Im Saalekreis existiert keine Bibliothek mit 30-40.000 zu betreuenden Einwohnern. 


\subsubsection{Fragenkomplex: Grundsatzfragen}

Das verwendete Bibliotheksmanagementsystem wurde in Frage $\mathbf{4}^{119}$ behandelt. Bibliotheksmanagementsysteme sind in den Öffentlichen Bibliotheken Deutschlands seit Ende der 1980er und im Laufe der 1990er Jahre eingeführt worden. Die meisten Bibliotheken leben seitdem mit der damals getroffenen Kaufentscheidung - ein Wechsel des Bibliotheksmanagementsystems findet sehr selten statt, da der komplette Arbeitsablauf auf das System abgestimmt ist. Ein Bibliotheksmanagementsystem aus dieser Zeit wird oft als ,integriertes Bibliothekssystem' oder einfach ,Bibliothekssystem' bezeichnet. Dieser Begriff „umschreibt dabei eine modular aufgebaute Software, die weitgehend alle Organisationsbereiche einer Bibliothek abdecken soll. Dies sind nach allgemeinem Verständnis mindestens die Erwerbung, Katalogisierung, Ausleihe, Nutzerdatenverwaltung und der Endnutzerkatalog. “120 Das Wort ,integriert' steht hierbei für die Verknüpfung der verschiedenen Komponenten auf Grundlage einer gemeinsam genutzten Datenbasis. Die unterschiedlichen Geschäftsgänge werden zwar meist in verschiedenen Modulen abgearbeitet, sie sind jedoch so verknüpft, dass Redundanzen in den Arbeitsabläufen vermieden werden können. Die Systeme nutzen eine lokale Datenbank in einem Client-Server-Modell. Einige Softwareanbieter stellen seit mehreren Jahren auch Hosting-Lösungen bereit. Im Einsatz sind verschiedene Varianten: Hosting mit Lizenzerwerb oder Mietvarianten im Modell von Software as a Service (SaaS) ${ }^{121}$. Die heute genutzten Systeme vereinen die üblichen Arbeitsvorgänge in Bibliotheken, die von der Bestellung über die Einarbeitung und Katalogisierung bis zur Ausleihe reichen. In dieser Arbeit wird daher der Begriff Bibliotheksmanagementsystem als Bezeichnung genutzt.

Die an der Umfrage teilnehmenden Bibliotheken verteilen sich auf 4 Bibliotheksmanagementsysteme. Zwei Bibliotheken setzen keine Software ein, eine Bibliothek nutzt eine Software, die nicht mehr weiterentwickelt wird. Es gibt in Deutschland kein Verzeichnis der eingesetzten Softwaresysteme, wodurch die Kommunikation der Bibliotheken untereinander erschwert ist. Obwohl die Arbeitsvorgänge nahezu identisch sind, findet jede Bibliothek ihren arbeitstechnisch eigenen Weg. Die in dieser Arbeit dargestellte Katalogisierungssituation der einzelnen Bibliotheken ist dafür ein Beispiel.

\footnotetext{
119 Frage 4: ,Welches Bibliotheksmanagementsystem setzen Sie in Ihrer Bibliothek ein? Gemeint ist die Software Ihres Ausleih- und Katalogsystems.'

120 Kemner-Heek 2012, S. 13.

${ }^{121}$ Variante des Cloud Computing, bei der Software und IT-Infrastruktur beim Dienstleister betrieben werden.
} 


\section{Verteilung der eingesetzten Bibliotheksmanagementsysteme}

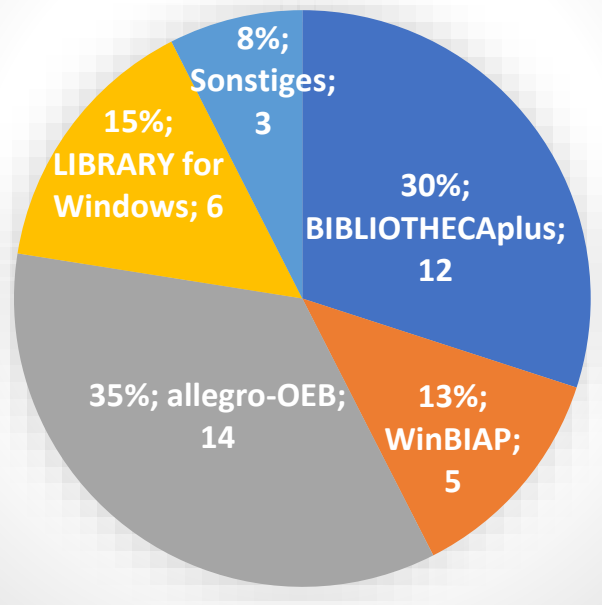

Abb.: 7 Prozentuale Verteilung der eingesetzten Bibliotheksmanagementsysteme der teilnehmenden Öffentlichen Bibliotheken in Sachsen-Anhalt $(n=40)$

Tab.: 4 Verteilung der eingesetzten Bibliotheksmanagementsysteme der teilnehmenden Bibliotheken in SachsenAnhalt nach Einwohnergruppen $(n=40)$

\begin{tabular}{|c|c|c|c|c|c|c|}
\hline $\begin{array}{c}\text { Einwohner } \\
\text { in } \\
\text { Tausend }\end{array}$ & $\begin{array}{c}\text { BIBLIOTHECA } \\
\text { plus }\end{array}$ & WinBIAP & allegro-OEB & $\begin{array}{c}\text { LIBRARY } \\
\text { for Windows }\end{array}$ & $\begin{array}{c}\text { Keine, bzw. } \\
\text { veraltete } \\
\text { Software }\end{array}$ & Gesamt \\
\hline bis 10 & 4 & 0 & 2 & 2 & 3 & 11 \\
\hline $10-20$ & 1 & 1 & 7 & 2 & 0 & 11 \\
\hline $20-30$ & 0 & 2 & 2 & 2 & 0 & 6 \\
\hline $30-40$ & 2 & 1 & 3 & 0 & 0 & 6 \\
\hline $40-50$ & 2 & 1 & 0 & 0 & 0 & 3 \\
\hline über 50 & 3 & 0 & 0 & 0 & 0 & 3 \\
\hline Gesamt & $\mathbf{1 2}$ & $\mathbf{5}$ & $\mathbf{1 4}$ & $\mathbf{6}$ & $\mathbf{3}$ & $\mathbf{4 0}$ \\
\hline
\end{tabular}

\subsubsection{Fragenkomplex: Ausstattung und Technik}

Mit dem Erfassen der Titeldaten in elektronischen Katalogen wurde nicht nur die Voraussetzung für eine automatisierte Ausleihverbuchung ermöglicht, sondern auch die Präsentation der vorhandenen Medien in einem öffentlich zugänglichen WWW-Katalog. Der sogenannte OPAC wird für die meisten Bibliothekssysteme als zusätzliches Modul angeboten. Der DBS-Vergleich der Bibliotheken Sachsen-Anhalts ${ }^{122}$ zeigt hier in den Jahren 2007 bis 2012 einen deutlichen Zuwachs. Haben 2007 noch 57\% aller Bibliotheken (42) keinen OPAC angegeben, so reduzierte sich diese Zahl 2012 auf 30 Bibliotheken (41\%). In den nächsten 5 Jahren konnte in nur einer weiteren Bibliothek ein OPAC eingerichtet werden. Die Umfrage ergab aus den Antworten zu

122 Siehe Tabelle DBS gesamt_DBS-Personal.xIsx auf beigelegter CD. 
Frage $\mathbf{5}^{123}$, dass mit 10 Bibliotheken $25 \%$ der beteiligten Bibliotheken ihren Nutzern keine Möglichkeit einer selbständigen Recherche, Verlängerung oder Vorbestellung außerhalb der Bibliotheksräume anbieten können. Die Präsentation im Internet und die Suchmöglichkeit nach den konkreten Angeboten der Bibliothek ist im Jahr 2019 eine Grundvoraussetzung, um von Einwohnern der Gemeinden gesehen und gefunden zu werden. Die Möglichkeit einer Implementierung eines OPAC oder Discovery-Systems sollte daher von jeder Bibliothek geprüft werden. Sinnvoll ist ein Zusammenschluss von mehreren kleineren Bibliotheken, um den finanziellen und personellen Aufwand zu reduzieren. Im Bundesland sollte diese Möglichkeit nochmals geprüft werden (siehe Fazit).

Wichtige Voraussetzung für eine Katalogisierung auf Basis einer funktionsfähigen Fremddatenübernahme ist der in Frage $\mathbf{1 0 ^ { 1 2 4 }}$ gestellte Sachverhalt, wer das System und die Hardware in der Bibliothek betreut. Bibliothekarinnen in Öffentlichen Bibliotheken verfügen oftmals nicht über ausreichend Kenntnisse, um Vorgänge wie webbasierte Übertragung von Metadaten, dabei verwendete Formate und Protokolle angemessen bewerten zu können und entsprechend konkrete Anforderungen an die mit der technischen Betreuung beauftragten Firmen weiterzugeben. Der Beruf des Systembibliothekars oder Bibliotheksinformatikers wird erst seit wenigen Jahren ausgebildet und kann auch nur in größeren Einrichtungen eingesetzt werden, die spezialisiertes Personal beschäftigen können. Ein falsches Berufsimage in der Gesellschaft, eine daraus resultierende Bewerbersituation, die zu wenig technikaffine Menschen in die Bibliotheken bringt, behindern derzeit ebenfalls eine modernere Bibliotheksarbeit. ${ }^{125}$

Das Personal der jeweiligen IT-Abteilungen der Gemeinden und Städte, bzw. der für die Kommunen arbeitenden IT-Firmen hat in der Regel ebenfalls keine BibliotheksinformatikAusbildung und beherrscht durch seine herkömmliche IT-Ausbildung die bibliotheksspezifischen Anforderungen nicht. Der Status der ,freiwilligen Selbstverwaltungsaufgabe ${ }^{126}$, den Öffentliche Bibliotheken in ihren Kommunen haben, erschwert finanzbedingt hierbei oft weitere mögliche Initiativen. Kleinere Bibliotheken werden meist von örtlichen IT-Firmen betreut.

\footnotetext{
${ }^{123}$ Frage 5: ,Setzen Sie für Ihre Bibliotheksnutzer einen Katalog/ OPAC bzw. ein Discovery-System ein?‘

${ }^{124}$ Frage 10: ,Wer betreut in Ihrer Bibliothek die Hardware und die Server?"

$125 \mathrm{Vgl}$. Schleihagen 2018, S. 470-471.

${ }^{126}$ Im Gegensatz zur ,pflichtigen Selbstverwaltungsaufgabe' kann jede Kommune entscheiden, ob und wie sie eine Bibliothek führt.
} 


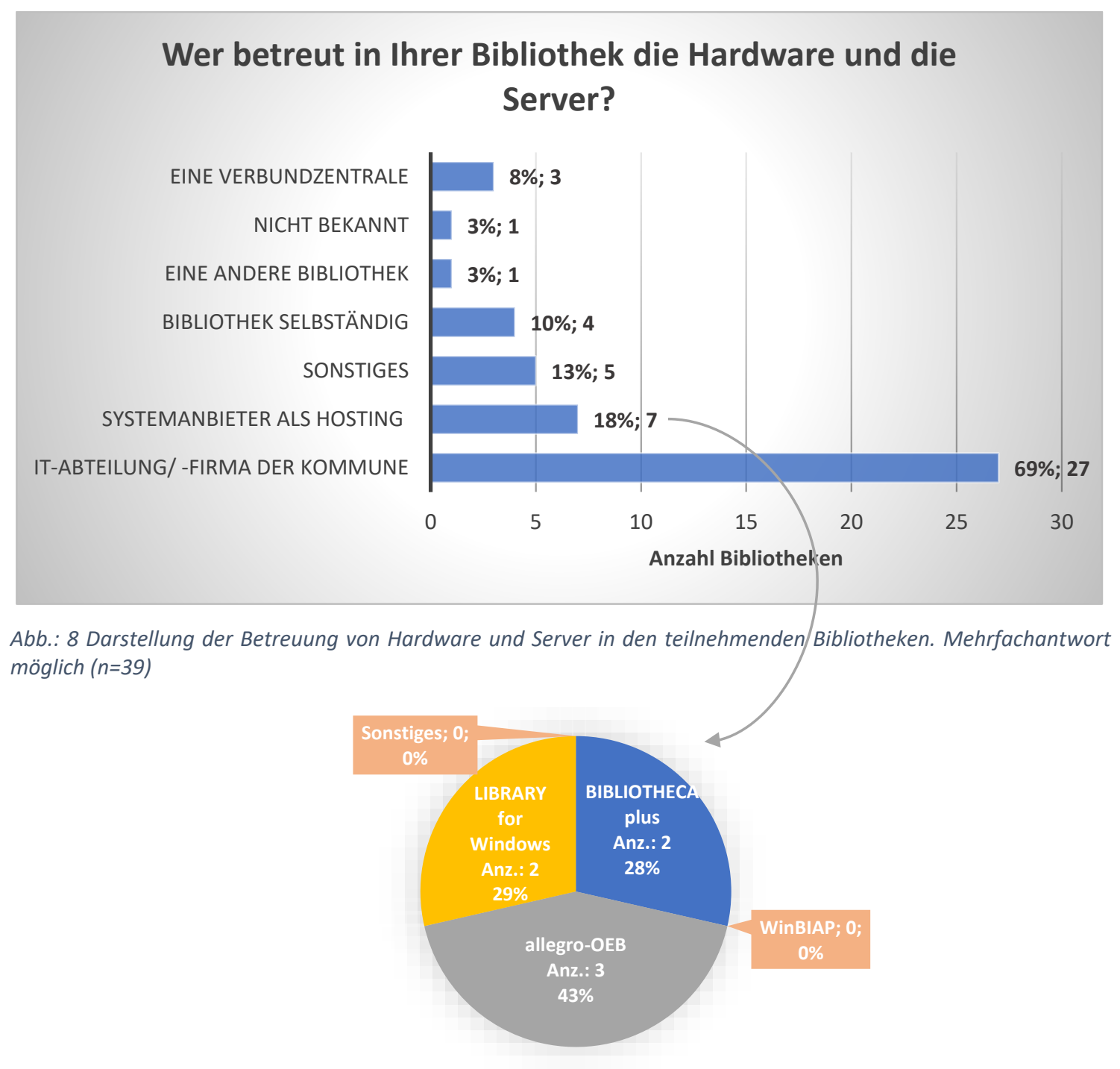

Abb.: 9 Verteilung der Bibliotheken mit Hosting des Bibliotheksmanagementsystems ( $n=7)$

$18 \%$ der beteiligten Bibliotheken (7) nutzen eine Hosting-Anwendung durch den jeweiligen Systembetreiber (OCLC und Fleischmann). Es sind außer den Großstadtbibliotheken Bibliotheken aller Größengruppen beteiligt. Unter Hosting verstehen die Systemanbieter neben der vorliegenden Software und automatisierten Updates auch die Datenhaltung in den jeweiligen Systemhäusern. Hosting bieten alle 4 Systemanbieter an. Insbesondere für kleinere Bibliotheken ist dies eine Variante, die auch für die Fremddatenübernahme beste Voraussetzungen bietet, da die Schnittstellen alle vom Anbieter bereitgestellt werden und keine interne Absprache mit der betreuenden IT vor Ort stattfinden muss, da die Anwendungen webbasiert sind (siehe Fazit). 


\subsubsection{Fragenkomplex: Katalogisierung /Fremddatenübernahme}

\section{Personal}

Der Einsatz von maßgeblich 2 Berufsgruppen in Öffentlichen Bibliotheken führt zu der Frage, welche der beiden Gruppen die Katalogisierung durchführt. Dazu wurde in Frage $\mathbf{3}^{\mathbf{1 2 7}}$ nicht nur die Anzahl des Personals in VZÄ verteilt auf die Berufsgruppen ,Fachbibliothekare', ,Fachangestellte, Bibliotheksassistentinnen ${ }^{128}$ und ,Sonstiges Personal ${ }^{129}$ abgefragt, sondern gleichzeitig eine Darunter-Zählung zum jeweiligen Einsatz in der Katalogisierung. Verwertbar sind die Antworten von 40 Bibliotheken. Die Gesamtsumme des Personals in den beteiligten Bibliotheken zeigt, dass in den Öffentlichen Bibliotheken Sachsen-Anhalts beide Berufsgruppen fast gleich häufig vertreten sind. Deutlich wird jedoch ebenfalls der große Anteil an ,Sonstigem Personal'. Unterschiede zeigen sich beim Einsatz in der Katalogisierung. Von 7 Bibliotheken wird angegeben, dass ,Sonstiges Personal' mit Arbeiten der Katalogisierung betraut ist - das entspricht 7\% des eingesetzten Personals (6,62 VZÄ). FAMIs katalogisieren zu 37\% (35,4 VZÄ) in 21 Bibliotheken. 56\% der Fachbibliothekarinnen sind in 31 Bibliotheken für die Katalogisierung zuständig (52,4 VZÄ). Der Gesamtanteil von katalogisierendem Personal liegt mit 94,4 VZÄ bei $41 \%$.

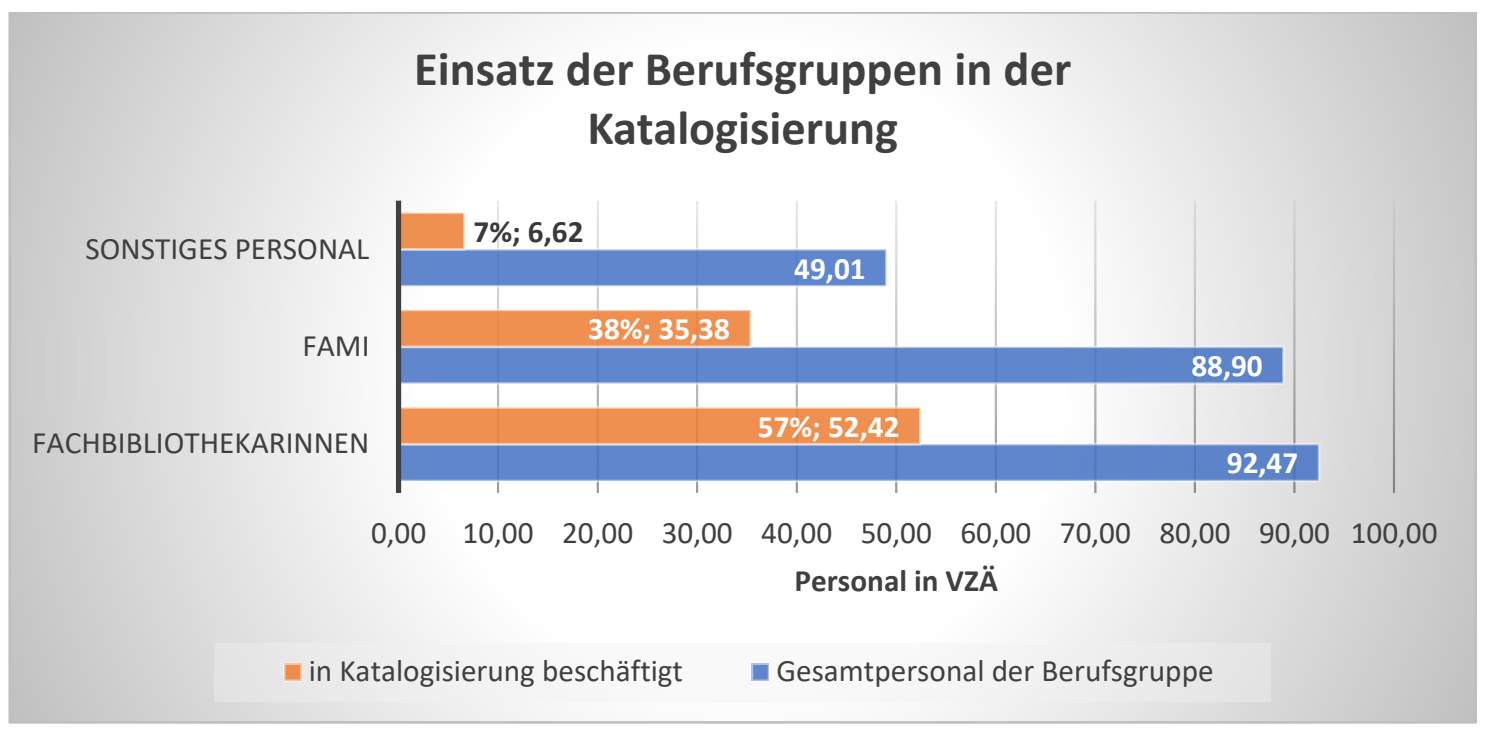

Abb.: 10 Gesamtpersonal in Sachsen-Anhalts Öffentlichen Bibliotheken nach Berufsgruppen, sowie anteiligem Einsatz in der Katalogisierung $(n=230,37)$

Die Begriffe der Berufsbezeichnungen und die Berechnungsgröße (VZÄ) wurden analog zur DBS verwendet. Aufgrund der hohen Personalschwankungen wurden sie dennoch abgefragt, da die letzte Statistik zum Erscheinungstermin dieser Arbeit mehr als ein Jahr zurück lag.

\footnotetext{
127 Frage: ,Wie viele Mitarbeiter/-innen sind in Ihrer Bibliothek beschäftigt?’

128 Um die Darstellung zu vereinfachen wird in der Arbeit vorwiegend der Begriff FAMI verwendet.

${ }^{129}$ Angelehnt an die Berufsbezeichnungen in der DBS.
} 
Eine Verteilung der Berufsgruppen auf die jeweiligen Einwohnergruppen zeigt eine deutliche Spezialisierung. Ab 40.000 zu betreuenden Einwohnern ist nur ausgebildetes Fachpersonal in der Katalogisierung eingesetzt. Mit abnehmender Anzahl der zu betreuenden Einwohner in den Gemeinden nimmt die Anzahl der Fachassistenten in der Katalogisierung zu. In Gemeinden unter 30.000 Einwohnern wird auch ,Sonstiges Personal' mit Katalogisierungsaufgaben betraut.

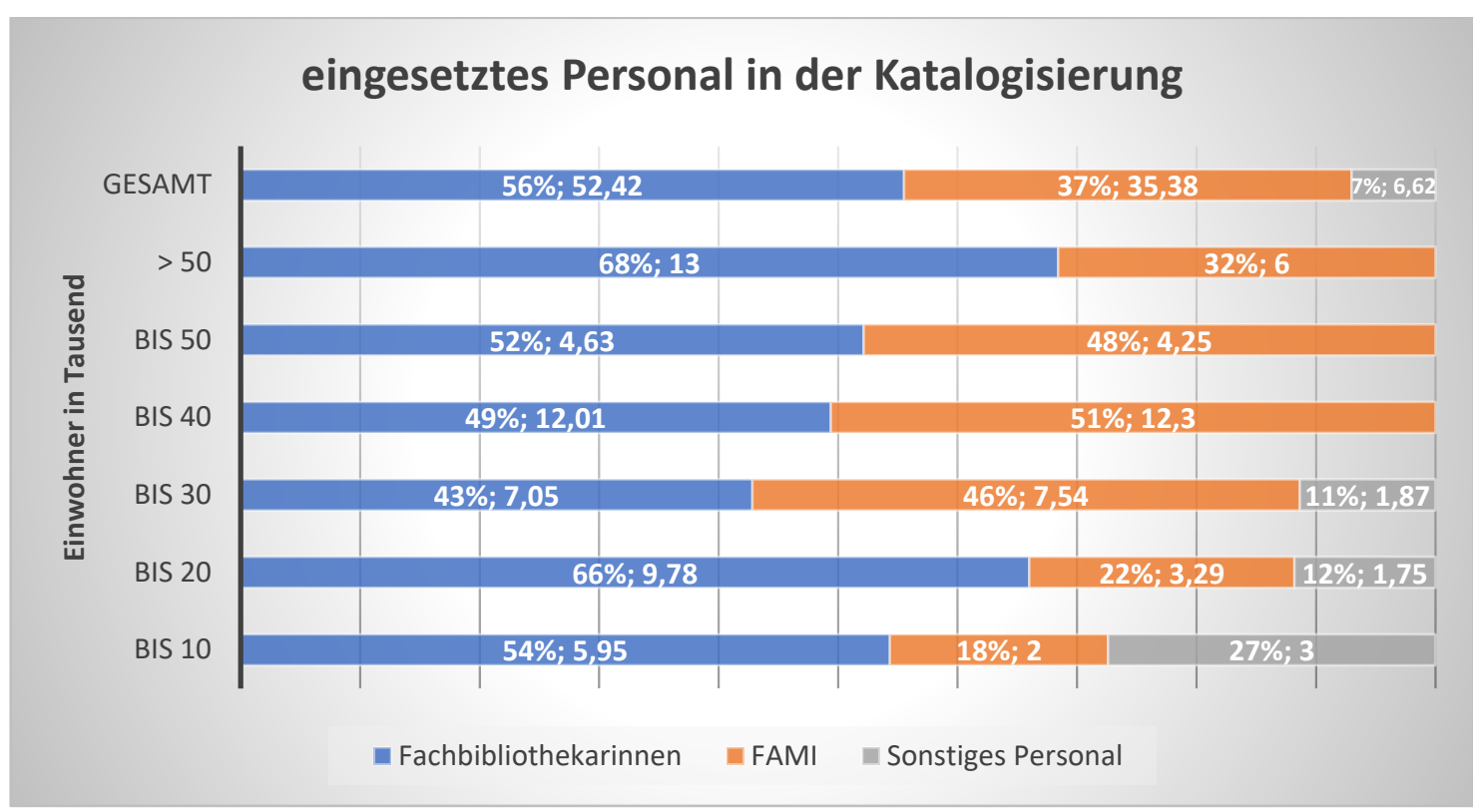

Abb.: 11 Verteilung der Berufsgruppen in der Katalogisierung innerhalb der Einwohnergruppen in VZ̈̈ $(n=94,42)$

Um Katalogisierung auf Grundlage von Fremddaten in Bibliotheken einrichten und durchführen zu können, verlangt es Kenntnisse in Datenformaten, Protokollen und Schnittstellen und setzt nicht zuletzt Wissen um zur Verfügung stehende Anbieter voraus. Die Beantwortung der in der Umfrage gestellten Fragen mit falschen Zuordnungen, die häufige Auswahl, ist mir nicht bekannt' oder ,dazu kann ich nichts sagen' bringt neben den konkret beantworteten Fragen klar zum Ausdruck, dass das technische Grundwissen bei vielen Mitarbeitern nicht oder nicht ausreichend zur Verfügung steht. Die Weiterentwicklung der Technik und die daraus resultierende Notwendigkeit der Anwendung in den Bibliotheken kann inhaltlich von großen Teilen des Personals noch nicht geleistet werden. Die bibliothekarische Fachpresse widmet sich mit Aussagen wie: die „fachlichen Anforderungen an das vorhandene Personal [können] nicht immer hinreichend erfüllt werden“130. oder „Wir brauchen extrem viel Fort- und Weiterbildung “131 diesem brisanten Thema - in der täglichen Arbeit wird Fortbildung zum Thema Katalogisieren in den Bibliotheken jedoch nicht kontinuierlich gelebt.

\footnotetext{
130 Schleihagen 2018, S. 470.

${ }^{131} \mathrm{Vgl}$. Münch 2014, S. 50.
} 


\section{Grundbedingungen}

Um den Stand der Katalogisierung einschätzen zu können, sind Aussagen über Grundbedingungen in den Bibliotheken notwendig. In Frage $\mathbf{8}^{132}$ werden die zu betrachtenden Voraussetzungen und Gegebenheiten abgefragt.

Die DNB stellt seit 2012 die Gemeinsame Normdatei (GND) in einer Online-Datenbank zur Verfügung. „Die GND verzeichnet die normierten Namen von Personen, Familien, Körperschaften, Kongressen, Geographika, Schlagwörtern und Titeln. “" ${ }^{133}$

Von den 37 antwortenden Bibliotheken nutzen 19\% (7 Bibliotheken) dieses Angebot. Sechs dieser Bibliotheken sind allegro-OEB-Anwender. Da es sich bei der 7. Antwort um eine sehr kleine Bibliothek handelt, wird diese Antwort eher angezweifelt. Es lässt sich schlussfolgern, dass von den vier verwendeten Bibliotheksmanagementsystemen nur allegro-OEB eine einfach nutzbare Möglichkeit der Datennutzung der GND anbietet.

Weiter abgefragt wurden die Katalogisierungsregeln durch Checkboxen in Mehrfachauswahl. Seit Ende 2015 sollten deutsche Bibliotheken ihre Titelaufnahmen auf der Basis von RDA anfertigen. ${ }^{134}$ Auch bei diesem Thema klafft die Spanne der durchgeführten Schulungen zwischen den Öffentlichen und Wissenschaftlichen Bibliotheken weit auseinander. In den Wissenschaftlichen Bibliotheken gab es Schulungspläne ${ }^{135}$, Mitarbeiter wurden zu Multiplikatoren ausgebildet. Die Verbundzentralen unterstützten und wurden einbezogen oder waren Initiator der Schulungen.

Anders in den Öffentlichen Bibliotheken: Es gibt keine zentrale Institution, die ein inhaltliches oder wirtschaftliches Interesse hat, um alle Bibliotheken in dieser Thematik auf den gleichen Stand zu bringen und ein Datum der Einführung festsetzen kann. Eine Weiterbildung der Fachstelle für Öffentliche Bibliotheken ist ein Ansatz - eine Fortführung und Begleitung dieser Thematik fehlt jedoch. Es bleibt in vielen Fällen der einzelnen Bibliothek überlassen, wann sie in welcher Form auf RDA umsteigt. Die eingesetzten Bibliotheksmanagementsysteme bieten teilweise erst seit 2018 die Möglichkeit, vollständig nach RDA zu katalogisieren. (siehe Anhang 3)

Aus Kenntnis der derzeitigen Situation wurde daher eine Auswahlmöglichkeit mit der Bezeichnung RAK-ÖB/ RDA-Mix angeboten. Hiermit wurde den Bibliotheken eine Antwortvariante angeboten, die ein System einsetzen, das noch nicht die kompletten RDA-

\footnotetext{
132 Frage 8: ,Katalogisierung - Schnittstellen, Normdateien (Frage nach: Nutzung von GND-Normdateien, eingesetzter Katalogisierungsregeln, Vorhandensein eines z39.50 Clients, genutztes Schnittstellenformat oderProtokoll, genutztes Datenformat.'

133 Gantert 2016, S. 193.

134 Wiesenmüller 2017, S. 52.

${ }^{135}$ Deutsche Nationalbibliothek 2018b. https://wiki.dnb.de/display/RDAINFO/Schulungen, (27.02.2019).
} 
Umfrage zur Katalogisierungssituation in Öffentlichen Bibliotheken Sachsen-Anhalts

Regeln umsetzen kann, aber auch den Bibliotheken, die in ihren internen Abläufen die Umsetzung auf die neuen Regeln noch nicht beendet haben. Es wurden 6 Mehrfachantworten gegeben. Bei 4 Antworten wurde der Eintrag unter RAK-ÖB gelöscht, da ein zusätzlicher bei RAKÖB/ RDA-Mix gesetzt worden ist. Ein 5. und 6. Doppeleintrag bei RAK-WB und RDA führte zu einer neuen Rubrik RAK-WB/ RDA-Mix. Die abgefragten Katalogisierungsregeln wurden in der folgenden Grafik dargestellt.

\section{Eingesetzte Katalogisierungsregeln der teilnehmenden Bibliotheken in Sachsen-Anhalt}

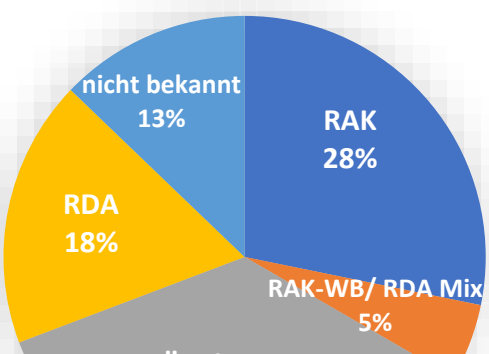

RAK-ÖB / RDA Mix

$36 \%$

Abb.: 12 Prozentuale Verteilung der verwendeten Katalogisierungsregeln

Deutlich wird, dass nur ein geringer Teil der Öffentlichen Bibliotheken bereits vollständig in RDA katalogisiert. Von den 7 Bibliotheken sind 5 allegro-OEB- und 2 BIBLIOTHECAplus-Anwender. Keine Anwenderbibliothek der beiden anderen Bibliotheksmanagementsysteme setzt komplett RDA ein.

Tab.: 5 Verteilung der eingesetzten Katalogisierungsregeln zu den eingesetzten Bibliotheksmanagementsystemen

\begin{tabular}{|l|c|c|c|c|c|c|}
\hline $\begin{array}{l}\text { Bibliotheks- } \\
\text { managementsystem }\end{array}$ & $\begin{array}{l}\text { nicht } \\
\text { bekannt }\end{array}$ & RAK-ÖB & $\begin{array}{l}\text { RAK-ÖB/ } \\
\text { RDA Mix }\end{array}$ & $\begin{array}{l}\text { RAK-WB/ } \\
\text { RDA Mix }\end{array}$ & RDA & $\begin{array}{l}\text { Gesamt- } \\
\text { ergebnis }\end{array}$ \\
\hline allegro-OEB & 1 & 3 & 5 & & 5 & 14 \\
\hline BIBLIOTHECAplus & 3 & 3 & 3 & 2 & 2 & 13 \\
\hline $\begin{array}{l}\text { LIBRARY for } \\
\text { Windows }\end{array}$ & & 3 & 3 & & & 6 \\
\hline WinBIAP & & 2 & 2 & & & 4 \\
\hline Sonstige & 1 & & 1 & & & 2 \\
\hline Gesamtergebnis & 5 & 11 & 14 & 2 & 7 & 39 \\
\hline
\end{tabular}

Weiterhin wurde das verwendete Schnittstellenformat bzw. -protokoll abgefragt, sowie die Frage gestellt, ob ein Z39.50 Client Bestandteil der Bibliotheksmanagementsoftware ist. 
Umfrage zur Katalogisierungssituation in Öffentlichen Bibliotheken Sachsen-Anhalts

Das Umfrageergebnis zeigt, dass mit 55\% 22 Bibliotheken einen Z39.50 Client vorhalten. 20\% (8 Bibliotheken) verneinen die Frage und 25\% können dazu keine Auskunft geben (10 Bibliotheken). Nur zwei von 39 Bibliotheken nutzen bereits den neuen Standard SRU.

Eingebunden in diesen Punkt wurde auch die Frage nach dem für Neuaufnahmen genutzten Datenformat. Hier wird deutlich, dass besonders in kleineren Bibliotheken die Bezeichnungen der Formate und Regelwerke nicht geläufig sind. Es wurden 46\% Antworten zu ,MAB2' (18 Bibliotheken), 26\% zu ,MARC21' (10 Bibliotheken) und 31\% zu ,anderen Formaten' gegeben (12 Bibliotheken). Die verbalen Begründungen lassen darauf schließen, dass die Frage nicht gut genug erklärt wurde.

Wurde als Antwort ,MARC21' gewählt, führte die Umfragelogik die Nutzer zu der Zusatzfrage $9^{136}$, ob die Altdaten konvertiert worden sind. In einer Bibliothek wurden die Altdaten in MARC21 konvertiert, 4 Bibliotheken haben eine Konvertierung verneint und viermal wurde ,nicht bekannt' ausgewählt. Seit 2008 löst das Datenformat MARC21 das frühere Datenformat MAB2 $\mathrm{ab}^{137} .2018$ sollten Katalogisierungsdaten daher weitestgehend in MARC21 vorliegen. Hier stellt sich jedoch die Frage: Ist das auch für die kleine Öffentliche Bibliothek notwendig, oder kann eine Konvertierung stattfinden, wenn die Bibliothek auf eine cloudbasierte Software umsteigt? Welcher Mehrwert bietet sich dem Nutzer, wenn die Bibliothek bisher nur über einen OPAC ohne weiterführende Funktionalitäten verfügt? Zu dieser Problematik sind die Mitarbeiter der Softwareanbieter gefragt worden.

Ein Anbieter konvertiert bei jedem Update die Daten. Bei den Bibliotheken, die ein Update durchgeführt haben, liegen demnach nun alle Daten in MARC21 und in RDA vor. Alle 3 anderen Anbieter führen dies nicht durch. Einheitlich wird hierbei geantwortet, dass eine Konvertierung der Altdaten nicht notwendig ist. Bestände der Öffentlichen Bibliotheken wechseln deutlich häufiger als die der Wissenschaftlichen Bibliotheken. Die alten MAB2/ RAK-Aufnahmen laufen in den Systemen einfach aus und werden mit der Löschung der Daten in wenigen Jahren verschwunden sein.

Ein Anbieter bietet eine "intellektuelle Anpassung" von MAB2 zu MARC21 an, in der es möglich gemacht wird, per Hand einzelne MAB2-Titel in MARC21 zu konvertieren, um dem Bibliotheksnutzer z.B. eine Recherche aller Filme mit einem konkreten Schauspieler zu ermöglichen.

\footnotetext{
${ }^{136}$ Frage 9: ,Wenn Sie MARC21 verwenden, wurden die alten Titelaufnahmen in MARC21 konvertiert?

137 Plassmann et al. 2011, S. 206.
} 
Umfrage zur Katalogisierungssituation in Öffentlichen Bibliotheken Sachsen-Anhalts

\section{Fremddatenübernahme}

Ein Hauptaugenmerk dieser Arbeit wurde auf die Datenübernahme gelegt. Abgefragt wurden die Arten der Fremddatenübernahme. Aus dieser Frage $\mathbf{1 5}^{\mathbf{1 3 8}}$ ergibt sich bei $\mathrm{n}=39$ Bibliotheken folgende Übersicht.

\section{Fremddatenübernahme in teilnehmenden Bibliotheken}

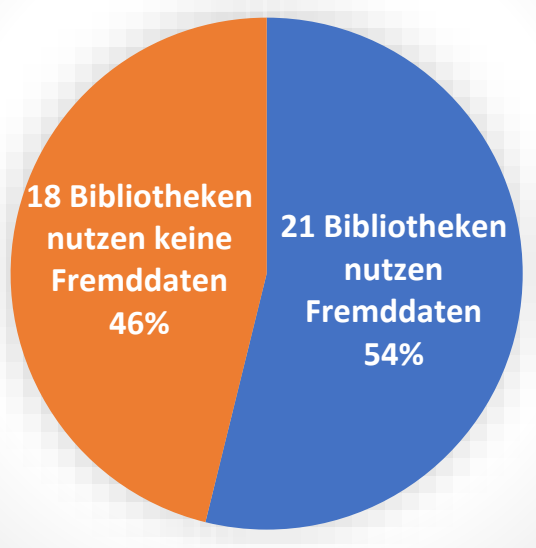

Abb.: 13 Prozentualer Anteil der teilnehmenden Bibliotheken mit und ohne Fremddatenübernahme (n=39)

Der Anteil von 18 Bibliotheken, die jegliche Katalogisate manuell erstellen ist mit $46 \%$ bemerkenswert hoch. Seit der Freigabe der Daten durch die DNB (2015) sollte der Anteil der Bibliotheken, die Fremddaten übernehmen, auch im Bereich der Öffentlichen Bibliotheken höher liegen. Erwartet wurde eine Nichtnutzung in den sehr kleinen Bibliotheken. In einer bundesweiten - von den Größengruppen vergleichbaren - Studie ${ }^{139}$ wurde ein Anteil von 16\% Bibliotheken ermittelt, die keinerlei Fremddaten nutzen. Diese Studie basiert auf einer repräsentativen Auswahl der Bibliotheken, weshalb in der vorliegenden Arbeit der absolute Anteil der kleinen Bibliotheken naturgemäß größer ist.

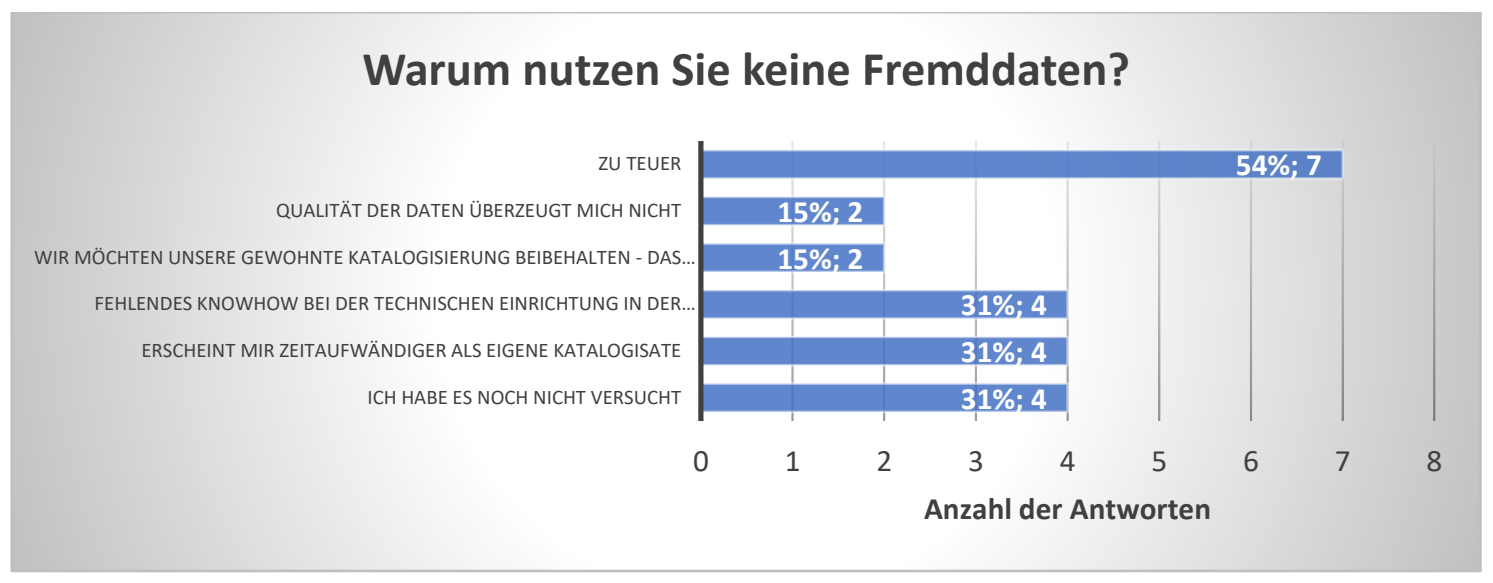

Abb.: 14 Auflistung der Gründe für Nichtnutzung von Fremddaten - prozentualer Anteil und Anzahl Antworten(n=13)

\footnotetext{
138 Frage 15: ,Welche Arten von Fremddaten bezieht Ihre Bibliothek?'

139 Woppowa 2018, S. 21.
} 
Umfrage zur Katalogisierungssituation in Öffentlichen Bibliotheken Sachsen-Anhalts

Die Beantwortung der Frage $21^{140}$ zeigt noch einmal, dass das Wissen über Fremddaten geschult werden sollte.

Ein Vergleich des zur Verfügung stehenden Medienetats der Bibliotheken in Sachsen-Anhalt anhand der DBS 2017 zeigt, dass die Möglichkeit einer automatisierten Datenübernahme für ein Datenabonnement der ekz.bibliotheksservice $\mathrm{GmbH}^{141}$ bei $41 \%$ der Bibliotheken in SachsenAnhalt nicht gegeben ist. Ihr Medienetat liegt mit bis $6000 €$ unter der Grenze, ab der eine Bibliothek selbst bei komplettem Abkauf des Etats bei der ekz ein Datenabonnement ${ }^{142}$ beziehen kann. Für diese Bibliotheken besteht die Möglichkeit, einzelne Datensätze pro recherchiertem Titel zu kaufen.

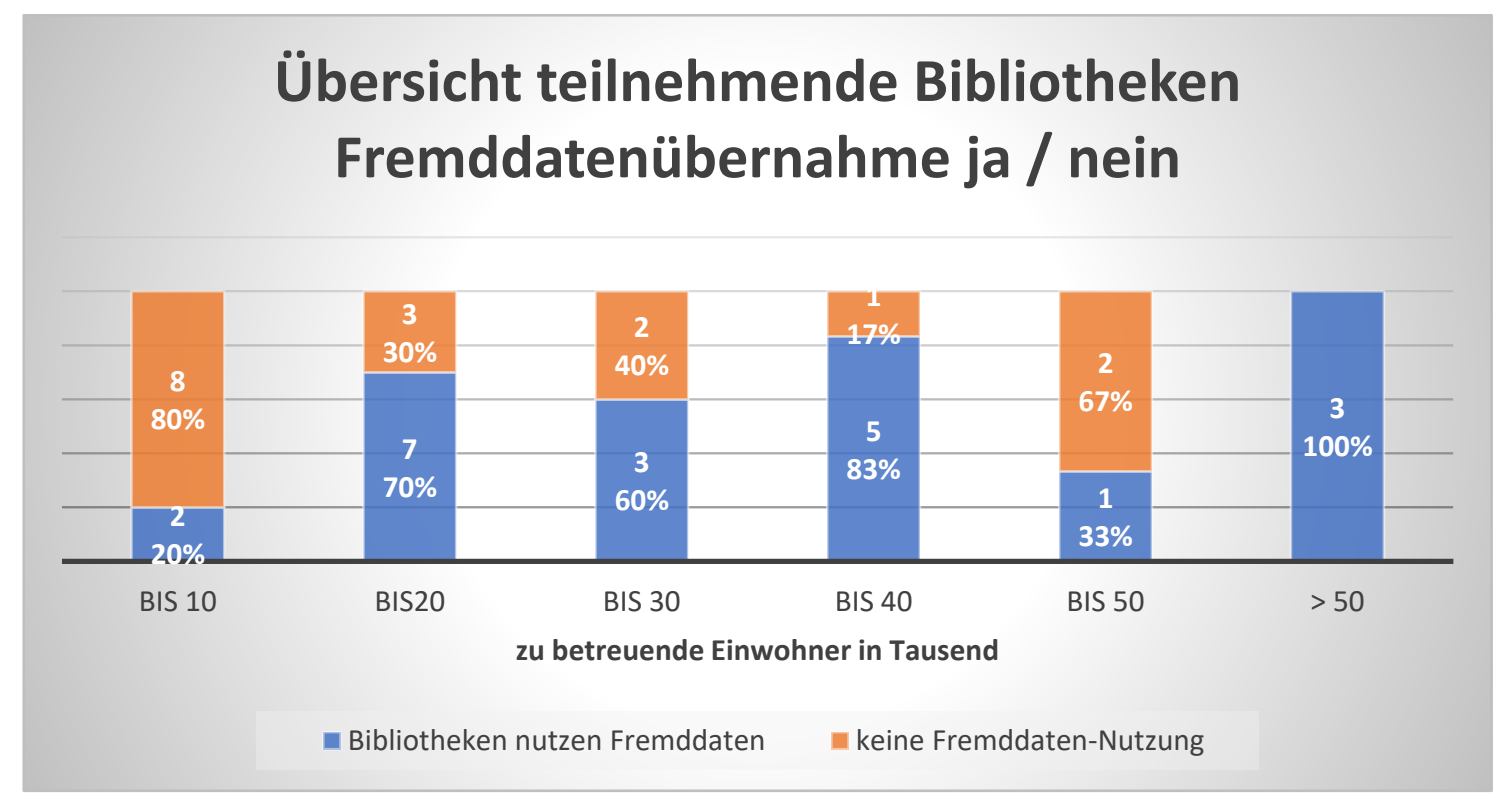

Abb.: 15 Anwendung von Fremddatenübernahmen der teilnehmenden Bibliotheken in Sachsen-Anhalt nach Einwohnergröße (n=39)

Eine Verteilung auf die Einwohnergrößen in Abbildung 15 belegt, dass der Anteil der Bibliotheken, die keine Fremddaten übernehmen, in den kleineren Bibliotheken besonders hoch ist. Aber auch der Prozentsatz der Bibliotheken der Kommunen mit 20 bis 50.000 Einwohnern weist einen hohen Prozentsatz aus. In der Übersicht wurden zwei Bibliotheken mit der Aussage ,dazu kann ich nichts sagen' mit in die Zählung der Bibliotheken ohne Fremddatennutzung gezählt, da diese Bibliotheken in Frage 16 angegeben haben, keine Fremddaten zu beziehen. Die Bibliothek einer Kommune über 40.000 Einwohner gibt an, dass im Jahr 2019 die Fremddatenübernahme geprüft wird. Durch die geringe Anzahl (3 Bibliotheken) in dieser Einwohnergruppe wirkt sich der prozentuale Anteil hier sehr stark aus. Auch von den 7

\footnotetext{
140 Frage 21: ,Warum nutzen Sie persönlich keine Fremddaten?"

141 ekz bibliotheksservice 2019a. https://www.ekz.de/ekz-bibliothekseinrichtung-ausstattung-service-und-medien/ (08.03.2019).

142 ekz bibliotheksservice 2019b. (24.02.2019).
} 
Bibliotheken, die ein Hosting der Softwarefirma nutzen wird von 3 Bibliotheken (42\%) die Möglichkeit, Fremddaten zu nutzen, nicht eingesetzt.

Mit $46 \%$ beziehen 18 Bibliotheken kostenfreie Fremddaten. Mit ebenfalls $46 \%$ nutzen ebenso viele Bibliotheken keine Fremddaten. Kostenpflichtige Katalogdaten werden von 6 Einrichtungen bezogen $\left(13 \%{ }^{143}\right)$. Diese Bibliotheken geben von Ihrem Medienetat bis zu $5 \%$ für kostenpflichtige Katalogdaten aus, wobei sich hierbei weder eine Tendenz zu einer Einwohnerzahlgruppe noch zu einem Bibliotheksmanagementsystem abzeichnet.

In Frage $16^{144}$ wurden die Bibliotheksleitungen nach den Anbietern und den eingesetzten Werkzeugen zur Fremddatenübernahme befragt. Mit 56\% antworteten 22 Bibliotheken inhaltlich und 44\% (17 Bibliotheken) mit der Antwort, wir nutzen keine Fremddaten'. Mit 50\% (11 Antworten) liegt die häufigste Nutzung bei der DNB, 41\% (je 9 Antworten) der Bibliotheken nutzen Fremddaten der Bibliotheksverbünde und der DIVIBIB, 36\% die der ekz (8 Nennungen). 18\% (4 Bibliotheken) nutzen das ZACK-Gateway und eine Bibliothek bekommt Daten von Hugendubel Fachinformation ${ }^{145}$. Mit ,Buch und Kunst ${ }^{146}$, der SpielTruhe ${ }^{147}$ (2 Nennungen) und amazon $^{148}$ ist die Auswahl,Sonstiges' mit 32\% von 7 Bibliotheken ausgewählt worden.

Mit Frage 19 wurde diese Abfrage auch den Katalogisiererinnen gestellt. Die prozentuale Verteilung auf die genutzten Angebote ändert sich nicht wesentlich. Die DNB-Daten werden mit 55\% von 27 Teilnehmerinnen genannt. EKZ, Bibliotheksverbünde und ZACK liegen in vergleichbaren Prozentwerten. Interessant ist die hohe Nutzung des GBV. Davon ausgehend, dass sich die Gewohnheiten der katalogisierenden Mitarbeiterinnen nicht einfach ändern lassen, kann durch diese Routine eine deutlich verbesserte Fremddatennutzung erwartet werden, wenn es gelingt, die großen Bibliotheken der teilnehmenden Bundesländer in eine aktuelle Abgleichroutine zu bringen.

Deutlich wird anhand von Tabelle 6 ebenfalls die Vielfalt der genutzten Angebote. Ein Prozentsatz von $24 \%$ und 12 Antworten für, ich nutze keine Fremddaten' ist jedoch wiederum ein Zeichen für die Notwendigkeit von Schulungen und Unterstützung bei der Thematik Fremddatenübernahme.

\footnotetext{
${ }^{143}$ Abweichende Prozentzahl durch Mehrfachnennung (Bezug von kostenpflichtigen UND kostenfreien Daten).

${ }^{144}$ Frage 16: ,Welche Anbieter von Katalogdaten bzw. welche Werkzeuge nutzen Sie in Ihrer Bibliothek zur Katalogisierung per Fremddatenübernahme?"

${ }^{145}$ Siehe: https://www.hugendubel.info/services/bibliotheken, (01.03.2019).

${ }^{146}$ Siehe: http://www.buk-stender.de/ (01.03.2019).

147 Siehe: https://www.spieltruhe.de/b2c_index.php (01.03.2019).

${ }^{148}$ Siehe: https://www.amazon.de/ (01.03.2019).
} 


\begin{tabular}{|l|c|c|}
\hline \multicolumn{3}{|c|}{$\begin{array}{c}\text { Antworten aller } \\
\text { Teilnehmerinnen }\end{array}$} \\
\hline Premddatenquellen & 27 & $55 \%$ \\
\hline DNB & 22 & $45 \%$ \\
\hline Bibliotheksverbünde & 20 & $41 \%$ \\
\hline GBV & 19 & $39 \%$ \\
\hline EKZ & 12 & $24 \%$ \\
\hline ich nutze keine Fremddaten & 10 & $20 \%$ \\
\hline Zack-Gateway & 7 & $14 \%$ \\
\hline jpc & 7 & $14 \%$ \\
\hline amazon & 5 & $10 \%$ \\
\hline KOBV/VÖBB & 3 & $6 \%$ \\
\hline Wikipedia & 3 & $6 \%$ \\
\hline SWB & 2 & $4 \%$ \\
\hline SpielTruhe & 2 & $4 \%$ \\
\hline BCS & 2 & $4 \%$ \\
\hline KVK & 1 & $2 \%$ \\
\hline Hugendubel & 1 & $2 \%$ \\
\hline Notenhändler & 1 & $2 \%$ \\
\hline Suchmaschinen & 1 & $2 \%$ \\
\hline BVB & &
\end{tabular}

\section{Manuelle Katalogisierung}

Die Reduzierung von Eigenkatalogisaten ist eines der grundlegenden Ziele der Bibliotheksverbünde, um durch Kooperation Bearbeitungszeiten zu minimieren. In Frage $\mathbf{2 2}^{149}$ wurde der prozentuale Anteil an manueller Katalogisierung abgefragt, um die Situation in Sachsen-Anhalt darstellen zu können. Bezogen auf die Hauptmediengruppen sollten die Antworten auf 3 Prozentgruppen ${ }^{150}$ verteilt werden. Hierbei wurden die rein manuellen sowie die per Kopieren und Einfügen erstellten Katalogisate als Schätzung abgefragt. Diese Frage wurde allen Teilnehmenden der Umfrage angeboten, da die Beantwortung auf dem Stand der konkreten Situation beruhen soll, die nur durch die jeweilige für die Mediengruppe katalogisierenden Personen in der Bibliothek eingeschätzt werden können.

\footnotetext{
${ }^{149}$ Frage 22: ,Wenn Sie die Gesamtanzahl der neu katalogisierten Medien des Jahres 2018 in den folgenden Mediengruppen einschätzen - wieviel Prozent haben Sie persönlich REIN MANUELL katalogisiert.'

150 1. Gruppe: 1-25\%, 2. Gruppe: 26-75\%, 3. Gruppe: 76-100\% manuelle Katalogisierung.
} 


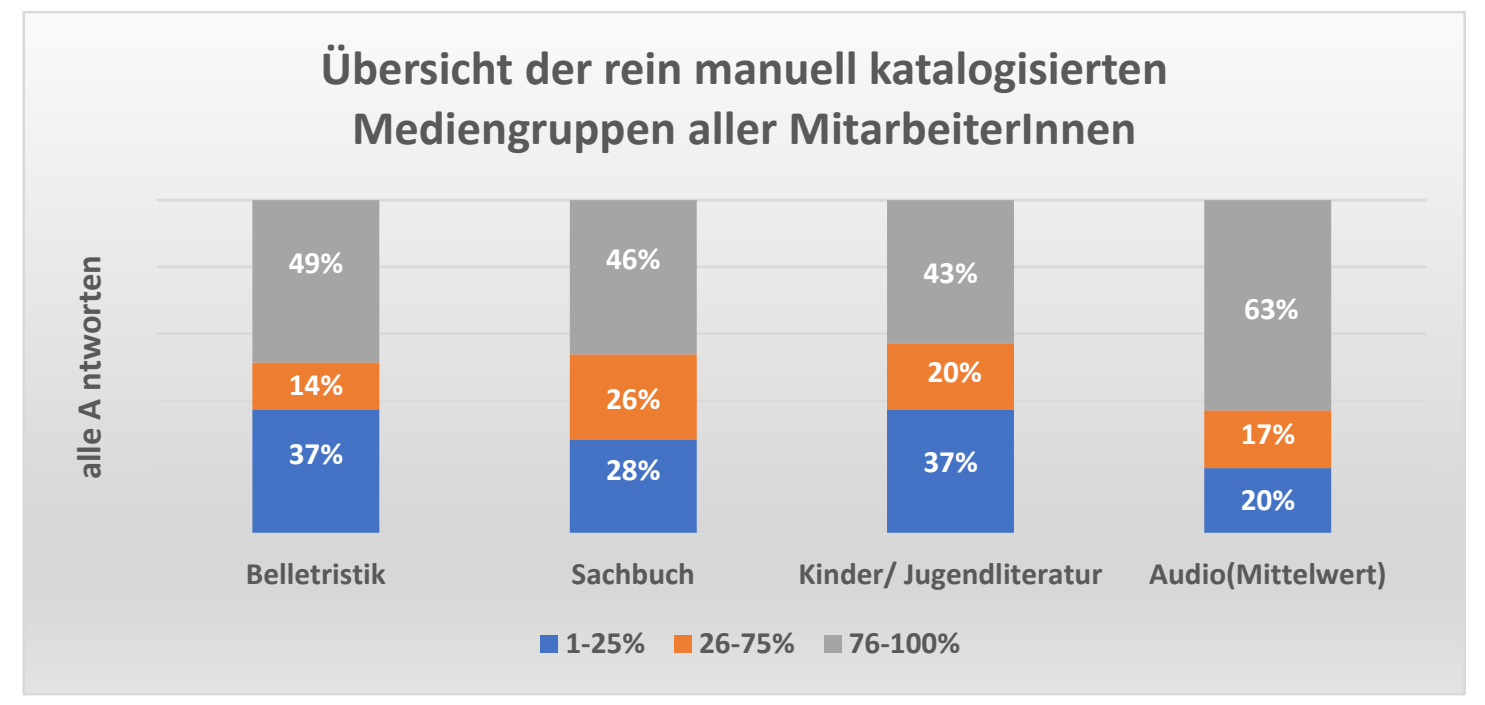

Abb.: 16 Anteile an manuell katalogisierten Mediengruppen aller Teilnehmenden der Umfrage ( $n=55)$

Durch die Einbeziehung der Antworten von Katalogisiererinnen und Bibliotheksleitung ist die Zuordnung zur jeweiligen Bibliothek nicht mehr gegeben. Dadurch liegt keine einheitliche Ausgangssumme an Teilnehmenden für die verschiedenen Mediengruppen vor. Die Tätigkeit der Katalogisierung ist in den größeren Bibliotheken auf verschiedene Kolleginnen verteilt, die sich auf Mediengruppen spezialisieren und in dieser Umfrage als Spezialisten geantwortet haben. Abbildung 16 zeigt, bezogen auf die Anzahl der zu bearbeitenden Medien, in der Summe am deutlichsten den reellen Stand im gesamten Bundesland, da die reine Anzahl der zu katalogisierenden Medien in den großen Bibliotheken die der kleinen Bibliotheken um ein Vielfaches übersteigt. Die Angaben beziehen sich jeweils auf 100\% der Antworten zur befragten Mediengruppe.

Durch alle Mediengruppen zieht sich eine hohe Prozentzahl der eigenen Katalogisate in der Gruppe der 76-100\% manuell, bzw. bei per Kopieren und Einfügen hergestellten Katalogisate. Die Mediengruppe Belletristik liegt mit $49 \%$ höher als Sachbuch (46\%) und Kinder- und Jugendliteratur.

Am deutlichsten ist der massive Anteil an eigenen Katalogisaten bei den audiovisuellen Medien. Die abgefragten Untergruppen, wie Hörbuch, CDs, DVDs oder BluRays wurden in Abbildung 17 zusammengezogen. $63 \%$ der Teilnehmenden schätzten ein, dass sie audiovisuelle Medien zwischen 76 und $100 \%$ manuell aufnehmen.

Eine detaillierte Auflistung der verschiedenen Erscheinungsformen der audiovisuellen Medien zeigt dabei keine deutlichen Ausreißer. 


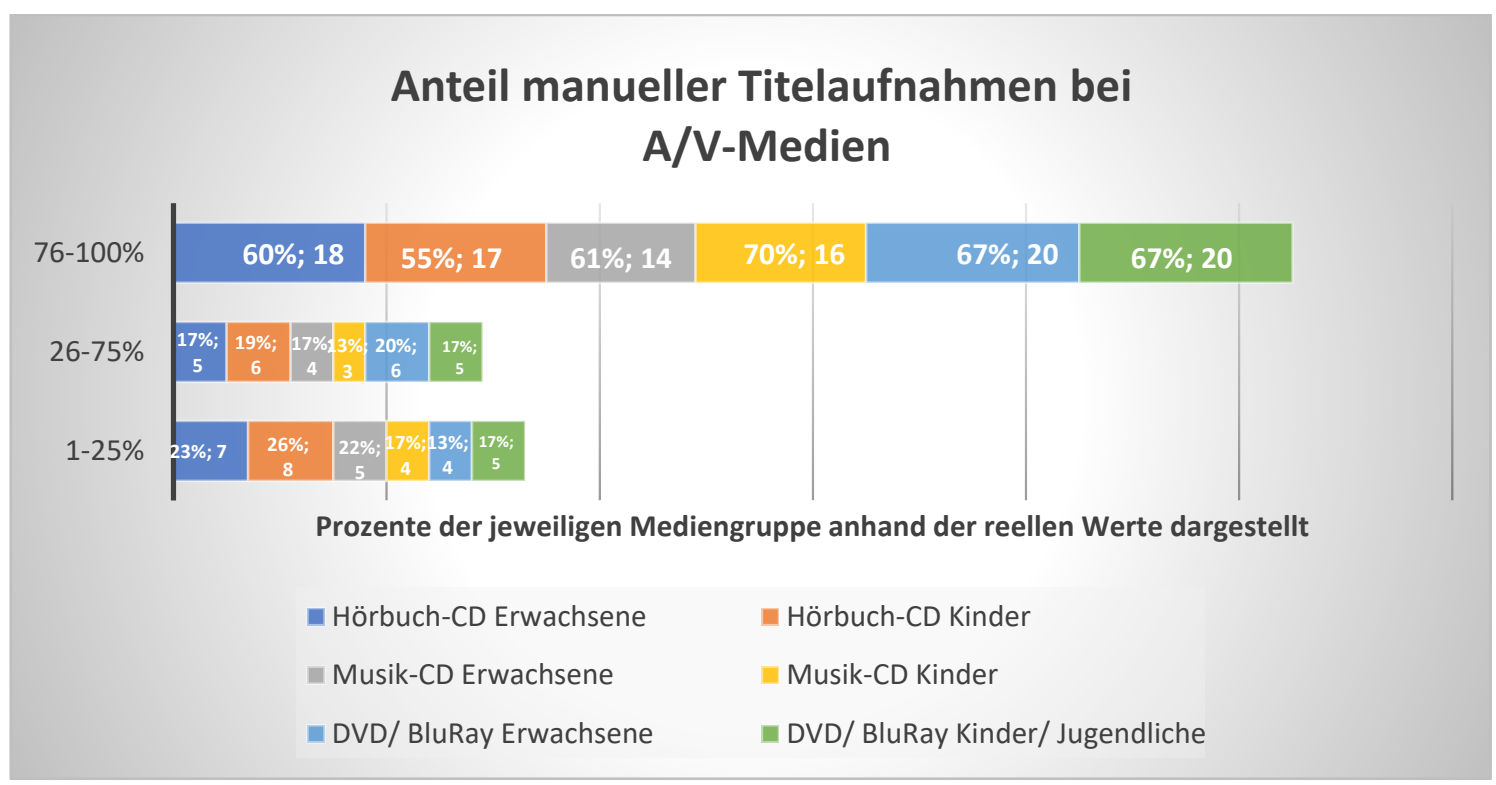

Abb.: 17 Anteile manueller Katalogisierung bezogen auf Varianten der AV-Medien

Besonders deutlich wird eine schlechte Datenlage bei DVD sowie bei Musik-CDs für Kinder. Die hohe Verbreitung von Hörbüchern scheint für eine etwas bessere Angebotssituation für Fremddaten zu sprechen.

Gegenübergestellt wurde in den Abbildungen 18 und 19, wie sich die prozentuale Verteilung verändert, vergleicht man alle Antworten mit den Antworten von Bibliotheken, die Fremddaten übernehmen. Erwartet wurde eine deutliche Abweichung. Dieser Vergleich zeigt die erwartete Abweichung bei den Printmedien ${ }^{151}$. Die tatsächliche Differenz in der Gruppe $1^{152}$ liegt bei $25 \%$. Das Beispiel der audiovisuellen Medien zeigt, dass sich diese Gruppe prozentual gar nicht verändert und mit jeweils $20 \%$ auch in den Bibliotheken mit Fremddatennutzung sehr niedrig liegt.

Das ekz-Geschäftsmodell ermöglicht den Bezug von Non-book-Meta-Daten ${ }^{153}$ ebenfalls erst ab einer festgelegten Umsatzgröße aus dem Bereich der Non-Book-Medien. Aus der Kombination der Antworten zu kostenpflichtigen Angeboten und Prozenten der manuellen Katalogisierung wird deutlich, dass dieses Angebot In Sachsen-Anhalt nicht genutzt wird oder die Anwender nicht teilgenommen haben.

\footnotetext{
151 Exemplarisch wurden hier die Medien aus der Gruppe Belletristik gewählt.

152 1-25\% der Titelaufnahmen werden manuell erfasst.

153 Die ekz fasst audiovisuelle Medien (CD/DVD/BD) unter dem Begriff ,Nonbook' zusammen.
} 


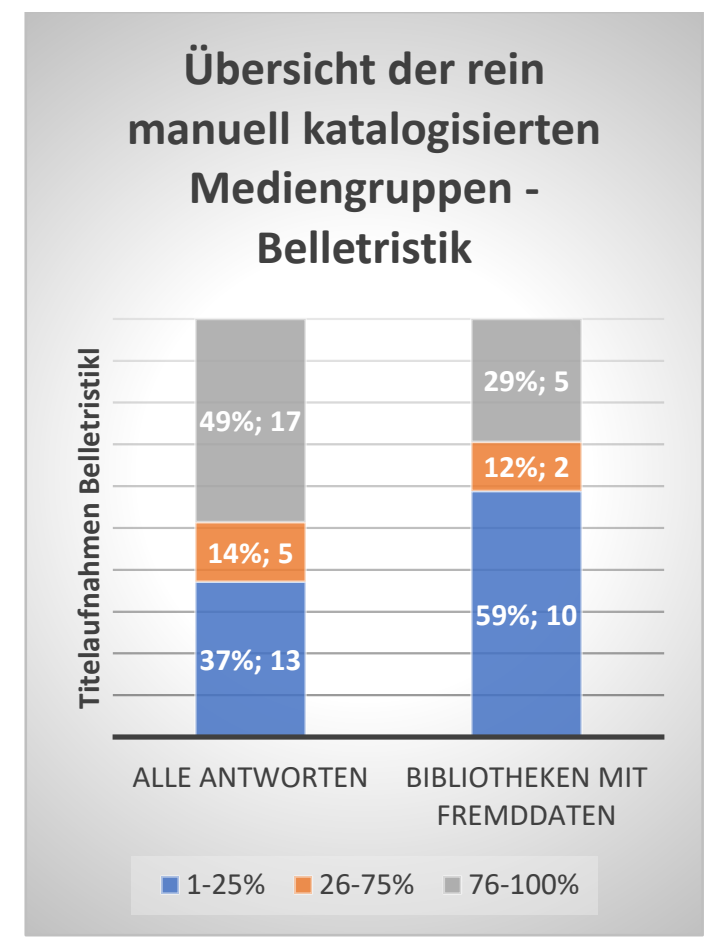

Abb.: 18 Beispieldarstellungen der anteiligen rein manuell erstellten Belletristik-Titelaufnahmen aller Befragten im Vergleich zu Bibliotheken, in denen Fremddaten eingesetzt werden (Prozent; Anzahl Antworten)

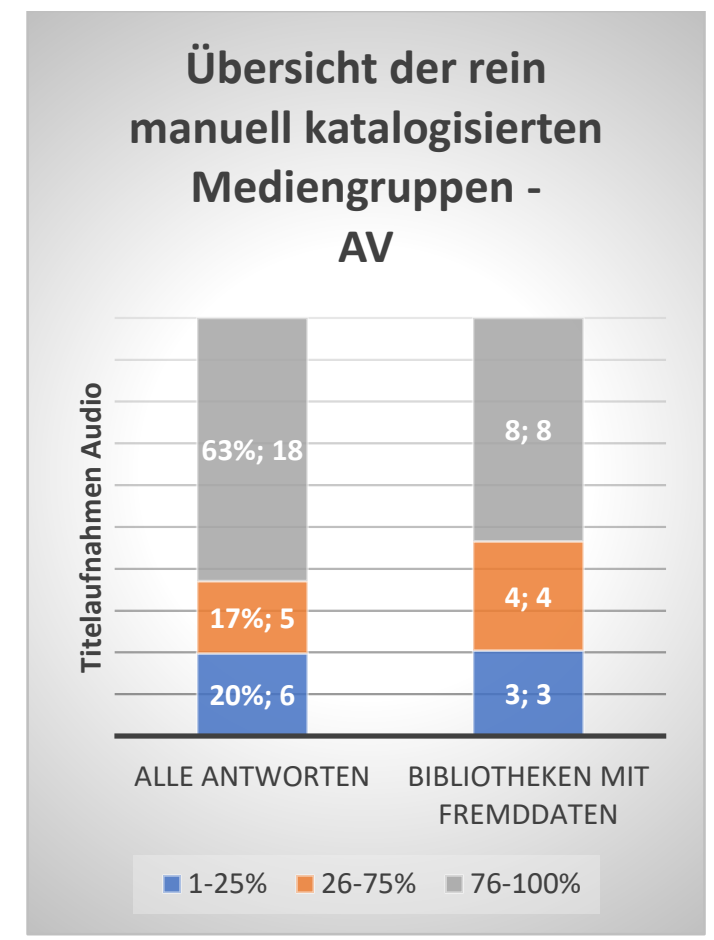

Abb.: 19 Beispieldarstellungen der anteiligen rein manuell erstellten Titelaufnahmen der audiovisuellen Medien aller Befragten im Vergleich zu Bibliotheken, in denen Fremddaten eingesetzt werden (Prozent; Anzahl Antworten)

Aus den Bemerkungen der Teilnehmenden geht weiterhin hervor, dass die speziellen Medien der Musikbibliotheken wie Musikdrucke und Noten besonders oft manuell katalogisiert werden, da keine Daten verfügbar bzw. nutzbar sind. Die Vereinigung der Musikbibliotheken hat auf der AlBM-Tagung 2017 die Problematik der kooperativen Katalogisierung thematisiert. Die als Ergebnis einer ,Umfrage zur RDA-Katalogisierung und Fremddatenübernahme' erwogene Zusammenarbeit mit der DNB konnte bisher nicht umgesetzt werden ${ }^{154}$.

Die Frage $\mathbf{2 0}^{155}$ ist die zweite Frage, die auch den Katalogisiererinnen gestellt worden ist. Bei der Fremddatenübernahme wählt die Mitarbeiterin nach persönlichen Vorlieben oder vorgegebenen Richtlinien der Bibliothek aus, welche Daten als Grundlage gewählt werden. Metadaten liegen zu gängigen Titeln in verschiedensten Formen in den unterschiedlichen Angeboten vor. Die Katalogisiererin entscheidet, ob und welche Daten übernommen werden. Die für die Suche ausschlaggebenden Kategorien wurden hier abgefragt und in Abbildung 20 dargestellt. Es antworteten 44 Katalogisiererinnen. Maßgebend ist die Antwort auf die Kategorie ,Schlagwort' gefallen. Mit 73\% bewerteten fast drei Viertel der Teilnehmenden diese Kategorie als wichtig für ihre Auswahl, eng gefolgt von der Annotation (66\%) und der Klassifikation (61\%). Sehen wir uns die Daten an, wird deutlich, warum das so ist.

\footnotetext{
154 Siehe: Wuthe 2017 http://www.aibm.info/wp-content/uploads/2017/09/2017-09-08_RDA_MusKat_VortragAlBM-2017.pdf (09.03.2019).

${ }^{155}$ Frage 20: ,Welche Katalogisierungs-Kategorien sind Ihnen bei einer Fremddatenübernahme persönlich wichtig (neben den Standardfeldern)?'
} 
Umfrage zur Katalogisierungssituation in Öffentlichen Bibliotheken Sachsen-Anhalts

Die Daten der DNB sind oft erst nach dem Kauf verfügbar. Schlagworte fehlen häufig komplett. Dies wird auch als Bemerkung einer Teilnehmenden konkret benannt: „Es ist sehr schade, dass die DNB kaum noch Schlagworte vergibt.". Die Klassifikation "KAB“ ist in den DNB-Daten nicht enthalten.

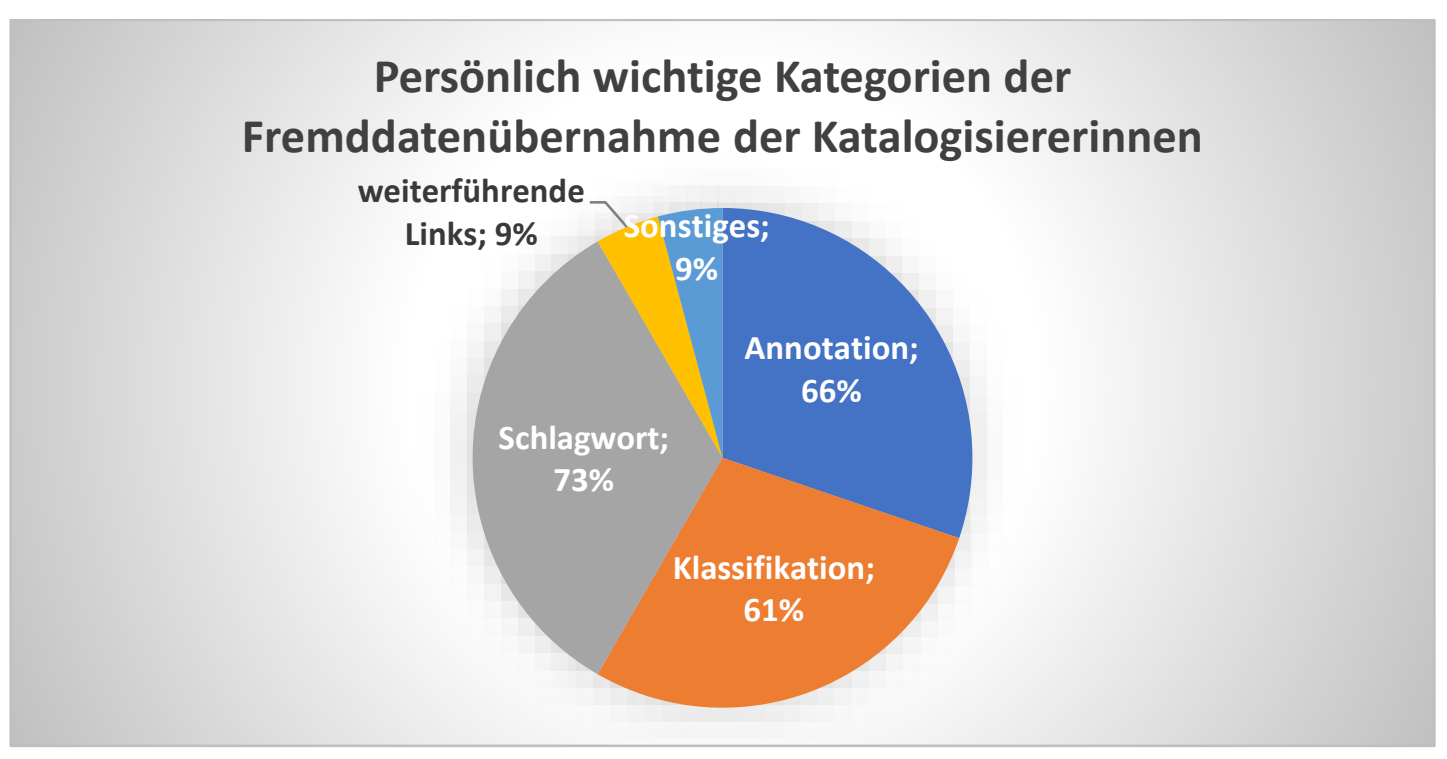

Abb.: 20 Prozentuale Darstellung der Wichtung der Katalogisierungskategorien bei der Fremddatenübernahme ( $n=44)$

\section{Katalogerweiterungen}

Qualitativ befragt wurden alle Teilnehmende in Frage $23^{156}$ nach ihrer persönlichen Einschätzung zu Katalogerweiterungen. Ergänzt wurde die Frage mit dem Hilfetext: ,Gemeint sind Coverabbildungen, BibTip, Links zu Wikipedia, Links zu Open-Access-Materialien oder Inhaltsverzeichnisse...'. Die Bewertung wurde mittels eines Schiebereglers in 10 Abstufungen von ,ich sehe es für unsere Nutzer nicht als hilfreich an' bis ,ich finde es sehr wichtig' abgefragt.

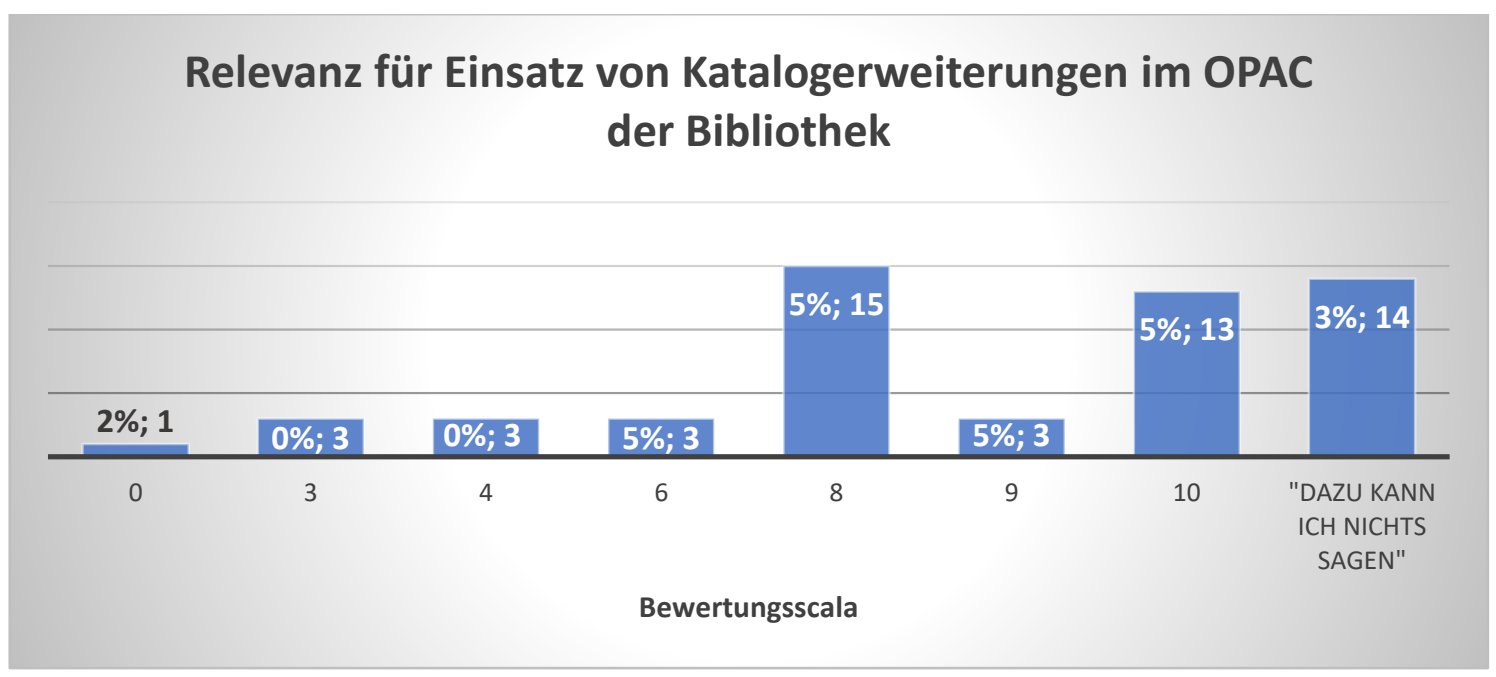

Abb.: 21 Verteilung der persönlichen Einschätzung der Relevanz der Katalogerweiterungen im OPAC ( $n=60)$

156 Frage 23: ,Für wie wichtig erachten Sie persönlich den Einsatz von Katalogerweiterungen (Catalog Enrichment) in Ihrem OPAC/ Discovery-System?’ 
Deutlich ist die Häufigkeit in der Auswahl der Einschätzung von $8-10$ Punkten. Von den 60 Teilnehmenden vergeben 15 (30\%) mit, 8’ Punkten der Katalogisierung eine hohe Wertigkeit. 3 (7\%) werten mit einer ,9‘ und 13 Teilnehmende (27\%) wählen die höchste Wertigkeit für diese Einschätzung aus. Eine Bewertung zwischen 3 und 7 Punkten vergeben 14 Mitarbeiterinnen. Nur eine Bibliotheksmitarbeiterin schätzt ein, dass Katalogerweiterungen im OPAC nicht wichtig sind - in dieser Bibliothek ist kein OPAC im Einsatz.

Die Bedeutung dieses Themas wird auch in der Antworthäufigkeit im Kommentarfeld, Möchten Sie dies begründen?'deutlich. 31\% der Teilnehmenden begründeten Ihre Entscheidung, was den größten Wert innerhalb der Kommentarfelder dieser Umfrage darstellt. Von den 11 inhaltlich relevanten Antworten gibt es 10 positive - hier meist in Bezug auf Cover, bessere Optik und verbesserte Suchmöglichkeit durch Inhaltsangaben. Zwei Teilnehmende geben den Nachteil der Ablenkung an - mehr Informationen überfordern evtl. den Nutzer: ,Links lenken meiner Meinung nach von ursprünglicher Absicht des Ausleihens und Medienfindens ab'.

Frage $24^{157}$ stellt die derzeitige Situation in den einzelnen Bibliotheken dar. Diese Frage ist gestellt worden, um im Fazit dieser Arbeit auch überregionale Bedürfnisse ermitteln zu können. Die einzelne Kollegin kann nur die Situation vor Ort einschätzen. Fortbildungen in der Katalogisierung sind in einer einzelnen Einrichtung (hier muss man die Großstadtbibliotheken ausnehmen) wenig zielführend. Beachten muss man auch, dass mit dieser Frage eventuell die eigene Leitungstätigkeit abgefragt und bewertet wird.

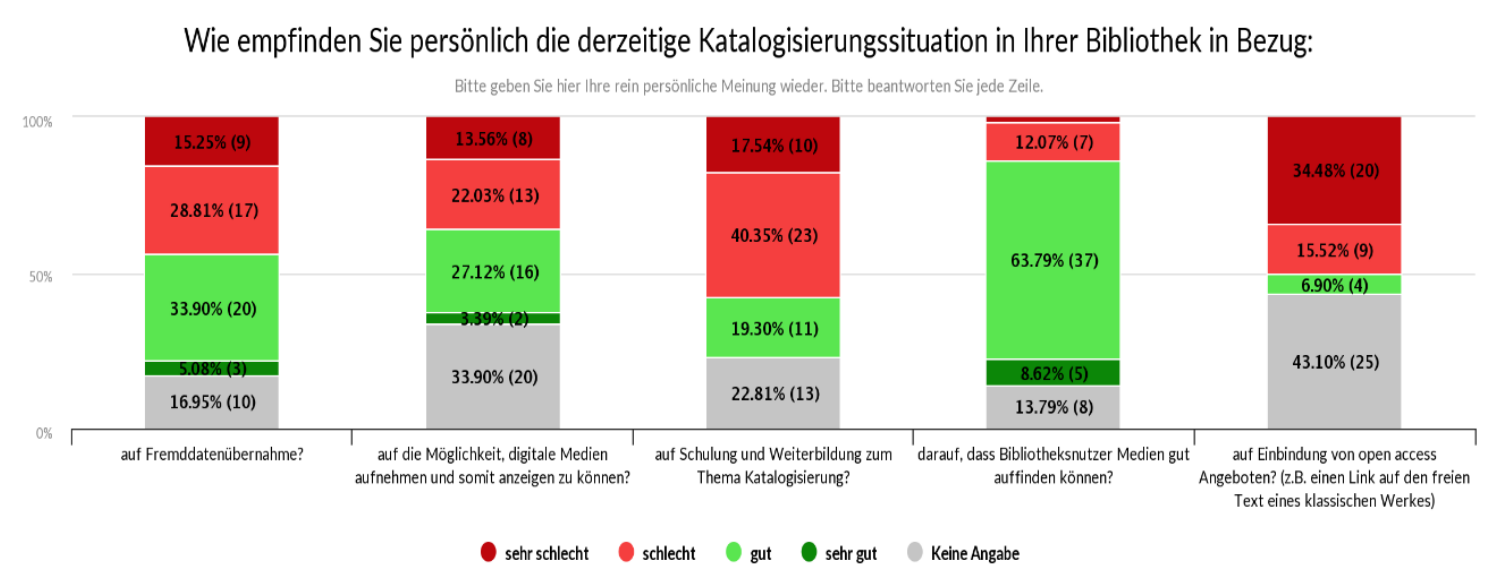

Abb.: 22 Verteilung der persönlichen Einschätzung der Katalogisierungssituation in der eigenen Bibliothek( $n=59)$

Auffallend sind 2 Dinge. Die Einschätzung, dass Bibliotheksnutzer die Medien gut auffinden können - ein Ergebnis a) einer guten sachlichen Erschließung und b) einer deutlich gekennzeichneten Aufstellung erreichte mit $72 \%$ positiven Bewertungen die höchsten

${ }^{157}$ Frage 24: ,Wie empfinden Sie persönlich die derzeitige Katalogisierungssituation in Ihrer Bibliothek?’ 
Ergebnisse. Beachten muss man hierbei, dass dies die Einschätzung des Bibliothekspersonals ist, nicht die der Bibliotheksnutzer.

Eine zweite interessante Schlussfolgerung kann man aus der Frage zu Angeboten von Open Access in den Bibliothekskatalogen ziehen. Diese Frage wurde von den meisten Teilnehmenden nicht beantwortet. Diese Reaktion lässt den Schluss zu, dass sich etliche Mitarbeiterinnen noch nicht mit dem Thema befasst haben. Auf der anderen Seite ist diese Frage auch diejenige mit der höchsten Prozentzahl an negativen Einschätzungen. 50\% der Befragten empfinden die Einbindung von Open-Access-Angeboten als schlecht oder sehr schlecht.

Die zentrale Forschungsthematik dieser Arbeit - die Fremddatenübernahme - wurde nur von $15 \%$ als sehr schlecht empfunden. Über $39 \%$ bewerten die Situation als gut oder sehr gut. Geht man von der prozentualen Nichtnutzung von $46 \%$ Fremddaten aus, ist diese Einschätzung nur mit Nichtkenntnis über die vorhandenen Möglichkeiten zu erklären und führt zu der nächsten Frage nach Weiterbildung. Folgerichtig ist diese Thematik mit 58\%, schlecht ' bzw. ,sehr schlecht ${ }^{\prime}$ im Vergleich der Fragen am negativsten beantwortet worden.

\subsection{Zusammenfassung und Überblick über die Umfrageergebnisse}

Grundgesamtheit für die Online-Umfrage zur Katalogisierungssituation der hauptamtlich geführten Öffentlichen Bibliotheken des Bundeslandes Sachsen-Anhalt stellen 75 Bibliotheken dar. Die Fragestellung wurde durch Filter und Logiken gesteuert. So begann die inhaltliche Hauptzielgruppe der Katalogisiererinnen erst ab Frage 19 - nachdem die jeweilige Bibliotheksleitung die organisatorischen, technischen, die grundlegenden inhaltlichen und personellen Belange beantwortet hatte. 66 auswertbare Teilnehmende und 63\% aller Bibliotheken haben an der Umfrage teilgenommen. Betrachtet man die Bibliotheken ab 20.000 zu betreuenden Einwohnern, liegt die Beteiligung sogar bei 85\%. Teilnehmende sind zusätzlich 25 Katalogisiererinnen, deren konkrete Arbeit abgefragt werden sollte.

Teilnehmende sind Bibliotheken aus Gemeinden von knapp 2.000 Einwohnern bis zu den beiden Großstädten Halle (Saale) und Magdeburg mit ca. 240.000 Einwohnern. Die nachfolgenden Werte beziehen sich jeweils auf die Teilnehmenden an der Umfrage.

Die Bibliotheken setzen 4 verschiedene Bibliothekmanagementsysteme ein:

- allegro-OEB (35\% - 14 Bibliotheken)

- BIBLIOTHECAplus (30\% - 12 Bibliotheken) - darunter alle 3 Großstadtbibliotheken

- LIBRARY for Windows (15\% - 6 Bibliotheken)

- WinBIAP (12\% - 5 Bibliotheken) 
8\% (3) der Bibliotheken setzen im Jahr 2019 kein oder ein nicht mehr gewartetes elektronisches Bibliotheksmanagementsystem ein, 25\% (10 Bibliotheken) können ihren Nutzern keinen OPAC und somit auch keinen Zugriff auf angebotene Medien, Selbstbedienungsfunktionen wie Verlängern oder Vorbestellen über das Internet anbieten.

Um die Katalogisierungssituation zu analysieren wurden Fragen zum Personaleinsatz, zur technischen Ausstattung und Betreuung sowie zu verwendeten Datenanbietern gestellt.

Bei $18 \%$ (7 Bibliotheken) ist durch ein Hosting abgesichert, dass das Bibliotheksmanagementsystem immer aktuell ist und die Medien- und Nutzerdaten auf einem gesicherten Firmen-Server liegen. Unter diesen 7 Bibliotheken befinden sich 3, die trotzdem keine Fremddaten beziehen.

Der Großteil der Bibliotheken (69\% -27 Bibliotheken) wird durch die städtische IT oder eine betreuende Firma der Stadt- oder Gemeindeverwaltung betreut, 10\% (4) sind für Hard- und Softwarewartung selbst verantwortlich.

In einer öffentlichen Bibliothek hat die katalogisierende Mitarbeiterin die volle Verantwortung für das zu erstellende Katalogisat, da eine kooperative Katalogisierung nicht vorhanden ist. Medien werden entweder manuell aufgenommen, ausgewählte Metadaten werden als Fremddaten importiert oder per Kopieren und Einfügen zusammengestellt. $42 \%$ des Gesamtpersonals der beteiligten Bibliotheken sind in der Katalogisierung tätig. Davon entfallen auf die einzelnen Berufsgruppen:

- $57 \%$ der Bibliothekarinnen,

- $40 \%$ der FAMIs sowie

- $14 \%$ des Sonstigen Personals

Abbildung 11 zeigt, dass die Spezialisierung mit der anwachsenden Einwohnerzahl zunimmt, aber auch, dass der prozentuale Anteil der eingesetzten FAMIs zu Bibliothekarinnen nahezu gleich ist.

Weiterhin wurde der Einsatz der seit 2015 in Deutschland eingesetzten Katalogisierungsregeln RDA sowie des Austauschformates MARC21 abgefragt. Haben bei dieser Thematik Wissenschaftliche Bibliotheken durch eine zentrale Katalogisierung Schulungen nutzen können und Multiplikatoren-Pläne entwickelt, stehen Öffentliche Bibliotheken hierbei jede für sich allein - in Abhängigkeit des Bibliotheksmanagementsystems - in der Verantwortung. 
Umfrage zur Katalogisierungssituation in Öffentlichen Bibliotheken Sachsen-Anhalts

RDA wird bisher in 7 Bibliotheken (18\%) eingesetzt. 41\% der Bibliotheken (16) arbeiten mit einem RDA/RAK-Mix. Die Umstellung ist dort noch nicht komplett vorgenommen worden. In 11 Bibliotheken (28\%) wird weiterhin ein reines RAK eingesetzt.

Der Einsatz von MARC21 in 10 Bibliotheken (26\%) bezieht sich zu 80\% auf 8 allegro-OEB Bibliotheken, 20\% auf 2 BIBLIOTHECAplus-Anwender. Hierbei wird, insbesondere bei kleineren Bibliotheken mit wenig technischem Know-how, die Abhängigkeit von und die Verantwortung des jeweiligen Anbieters des Bibliotheksmanagementsystems deutlich.

Diese Verantwortung besteht ebenso beim Angebot einer einfach durchzuführenden Fremddatenübernahme.

Im Ergebnis der Umfrage wurde deutlich, dass mit 18 Bibliotheken 46\% keine Fremddaten übernehmen. Mit Freigabe der Daten einiger Bibliotheksverbünde und der DNB unter einer CCOLizenz sollten diese Prozentzahlen höher liegen.

Bibliotheken, die Fremddaten nutzen, verwenden zu 61\% DNB-Daten (11 Bibliotheken), je 40\% (9 Bibliotheken) nutzen Daten der Bibliotheksverbünde und Daten der DIVIBIB und mit 36\% (8 Bibliotheken) Daten der ekz. 22\% der Bibliotheken (4) nutzen für die Fremddatenübernahme das ZACK-Gateway. Weiterhin werden die Angebote von Buch und Kunst, Spieltruhe, amazon und Hugendubel Fachinformation zu geringen Prozentwerten aufgelistet.

Die Frage nach der prozentualen Verteilung der rein manuell katalogisierten Medien auf die verschiedenen Mediengruppen brachte ein deutliches Problem bei der Übernahmemöglichkeit von audiovisuellen Medien zutage. Diese Frage wurde allen Teilnehmenden gestellt, sie ist somit nicht mehr auf Bibliothekssysteme oder Einwohnerklassen rückverfolgbar, stellt jedoch durch die Anzahl der Antworten ein realistisches Bild der Gesamttitelaufnahmen des Bundeslandes dar. 63\% der Titelaufnahmen in den Mediengruppen dieses Bereiches werden manuell erfasst. Die Werte der Bereiche Kinder- und Jugendbuch (43\%), Sachbuch (46\%) und Belletristik (49\%) liegen ebenfalls hoch, jedoch zeigt sich hierbei, dass mehr Daten zur Übernahme zur Verfügung stehen.

Besonders hoch ist der manuelle Aufwand in den Musikbibliotheken. Musikbibliotheken haben in ihrer AIBM-Sitzung 2017 diesen Sachverhalt thematisiert. Das anvisierte Ziel einer separaten Zugriffssicht und einer Kooperation mit der DNB konnte noch nicht erreicht werden.

Ermittelt wurde weiterhin die Priorisierung der Angebotsauswahl in Hinblick auf Kategorien. Drei Viertel der Katalogisiererinnen (32) wählten die Kategorie ,Schlagwort' für sie als am 
Umfrage zur Katalogisierungssituation in Öffentlichen Bibliotheken Sachsen-Anhalts

wichtigsten bei der Datenübernahme, gefolgt von einer Annotation (66\% - 29 Katalogisiererinnen) und der Klassifikation (61\% - 27 Katalogisiererinnen). Weiterführende Links wurden in dieser Frage mit 9\% von 4 Teilnehmenden als wichtig eingestuft. In der folgenden Frage wurde explizit nach dem persönlichen Standpunkt zu Katalogerweiterungen gefragt. 52\% der 60 Teilnehmenden geben mit 8-10 Punkten dem Catalog Enrichment eine besonders hohe Wertigkeit und begründen dies auch verbal mit einer verbesserten Optik durch Coverabbildungen oder der Möglichkeit, inhaltliche Relevanz eines Treffers auf eine Suchanfrage durch das Vorhalten von Inhaltsverzeichnissen prüfen zu können.

Kritisch wird die Überflutung von Informationen bewertet, da insbesondere von älteren Nutzern und Nutzerinnen das Wesentliche nicht sofort erfasst werden kann.

Qualitativ befragt wurden die Teilnehmenden nach ihrer persönlichen Einschätzung der Katalogisierungssituation in ihrer jeweiligen Einrichtung. 39\% der Teilnehmenden bewerteten die Situation als gut oder sehr gut. Bei $46 \%$ Nichtnutzung von vorhandenen Fremddaten lässt sich hieraus eine Unkenntnis der Angebotssituation schlussfolgern, was sich durch die negativste Beantwortung von 58\% (33 Teilnehmende), schlecht' oder ,sehr schlecht' bei der Frage nach Schulung und Weiterbildung zum Thema ,Katalogisierung' zum Ausdruck kommt. Das befragte Bibliothekspersonal schätzt die Auffindbarkeit von Medien in ihrem Katalog mit $72 \%$ (43 Antworten) positiven Bewertungen als positivste der Antworten ein. 


\section{Expertenbefragung}

\subsection{Auswahl der Experten}

Ein Ergebnis der Umfrage war das eingesetzte Bibliotheksmanagementsystem. Die Antworten ergaben eine Verteilung auf 4 Systeme:

- $\quad$ allegro-OEB - Büchereizentrale Niedersachsen ${ }^{158}$ (14 Bibliotheken)

- BIBLIOTHECAplus - OCLC ${ }^{159}$ (12 Bibliotheken)

- $\quad$ LIBRARY for Windows - Fleischmann Software Vertriebs $\mathrm{GmbH}^{160}$ (6 Bibliotheken)

- WinBIAP - datronic IT-Systeme GmbH \& Co. KG ${ }^{161}$ (5 Bibliotheken)

Der Einsatz von Bibliotheksmanagementsystemen erfolgt in Öffentlichen Bibliotheken nach individuell verfassten Ausschreibungen. In der Regel besteht die Zusammenarbeit mit dem Softwareanbieter für eine längere Zeit. Es liegen keine allgemein gültigen Vorgehensweisen oder wissenschaftliche Analysen zum Einsatz verschiedener Bibliotheksmanagementsysteme in Öffentlichen Bibliotheken vor. Um die in dieser Arbeit gestellte Forschungsfrage mit den jeweils technischen Möglichkeiten der Systeme abgleichen zu können, wurde als Mittel der Wahl eine Expertenbefragung mit Vertretern der eingesetzten Bibliotheksmanagementsysteme durchgeführt. Für die Expertengespräche ${ }^{162}$ wurden Mitarbeiter der jeweiligen SoftwareFirmen, bzw. im Fall von allegro-OEB der Büchereizentrale Niedersachsen angesprochen. Den Personen wurde der Fragebogen, sowie eine Interviewfreigabe vorab per Mail zugesandt. Die Gespräche wurden in Form von Telefoninterviews durchgeführt. Es handelt sich um Gespräche, denen ein konkreter Fragebogen mit überwiegend geschlossenen Fragen zugrunde lag, in denen Sachverhalte zu der jeweiligen Software abgefragt worden ist. Die Ergebnisse sind kein separater inhaltlicher Bestandteil der Arbeit im Sinne von qualitativen Interviews, sondern sollen anhand der konkreten Antworten die Möglichkeiten von Fremddatenübernahmen in den Bibliotheken verdeutlichen. Es wurde eine Kodierung der Antworten mit dem Programm MaxQda ${ }^{163}$ durchgeführt. Die Verschriftlichung der Antworten erfolgte in Form einer Antwort-Tabelle (siehe Anhang 3) Eine auf den Forschungsschwerpunkt bezogene Auswertung der einzelnen Systeme mit Ergebnissen der Onlineumfrage erfolgt für jedes System in Abschnitt 8.

Die Interviews liegen als mp3-Datei als prüfungsrelevanter Nachweis vor.

\footnotetext{
158 Büchereizentrale Niedersachsen 2019 https://www.bz-niedersachsen.de/allegro-oeb.html (13.03.2019).

159 OCLC 2019a. https://www.oclc.org/de/bibliotheca.html (13.03.2019).

${ }^{160}$ Fleischmann Software Vertriebs GmbH 2019.https://www.fleischmann.org/ (13.03.2019).

161 datronic IT-Systeme GmbH \& Co. KG o.J http://www.datronic.de/Bibliothekssysteme/WinBIAP (13.03.2019).

162 Nach: Meuser und Nagel, 2009, S. 443 - Personengruppen, die über einen privilegierten Zugang zu Informationen verfügen.

${ }^{163}$ VERBI GmbH 2019 https://www.maxqda.de/ (27.02.2019).
} 


\subsection{Zusammenfassung der Expertenbefragung}

Die Expertenbefragung umfasste jeweils elf konkret ausformulierte Fragen. Die Fragen lagen dem Interviewpartner vor und wurden vorab noch einmal vorgelesen.

Ziel der Befragung war es, den derzeitigen Stand der jeweiligen Software in Bezug auf Fremddatenübernahme, Umsetzung des Formates MARC21 und der Umsetzung der neuen Katalogisierungsregeln RDA festzustellen, um diesen mit den Antworten der Onlinebefragung abgleichen zu können.

Feststellbar ist, dass die Einführung der neuen Anforderungen zeitlich sehr unterschiedlich verlief und noch verläuft. Lediglich für allegro-OEB sah sich der Anbieter schon 2015 gezwungen, die neuen Technologien konsequent umzusetzen und den Bibliotheken zur Verfügung zu stellen. Die Software WinBIAP bietet mit der neuen Version eine komplette Nutzung. LIBRARY for Windows bietet die Abfrage-Möglichkeit über SRU an - ein Einspielen ist über eine Importdatei möglich. Hauptsächlich wird jedoch Z39.50 genutzt. BIBLIOTHECAplus bietet Importmöglichkeiten von MARC21 - jedoch noch keine SRU-Schnittstelle an.

RDA ist in allen 4 Bibliotheksmanagementsystemen implementiert und kann in den für Öffentliche Bibliotheken benötigten Feldern genutzt werden. Insbesondere die Möglichkeit, über SRU an Fremddaten zu gelangen und diese automatisiert einspielen zu können ist noch nicht in allen Systemen vollständig implementiert.

Auf die bestehende Frage, ob Bibliotheken ihre in MAB2 aufgenommenen Altdaten in MARC21 konvertieren sollten, gab es aufgrund der jeweils bestehenden internen Formate eine einheitliche ablehnende Meinung. Die Daten können sowohl in MAB2 als auch in MARC21 in der Software in die jeweiligen Internformate importiert und in den meisten Fällen auch exportiert werden. Eine Notwendigkeit zur Konvertierung bestehe daher nicht. Im Rahmen der Arbeit einer Öffentlichen Bibliothek liegen Bestandsdaten nicht so lange im jeweiligen Katalog vor, die hohe Erneuerungsquote ermöglicht durch den kontinuierlichen $\mathrm{Zu}$ - und Abgang hier eine Art ,natürliche Bereinigung' der Datenbank. 


\section{Möglichkeiten der kostenfreien Datenübernahme}

Aufgelistet und beschrieben werden in diesem Kapitel die derzeit zur Verfügung stehenden Anbieter, bzw. Werkzeuge, die für eine kostenfreie Fremddatenübernahme zur Verfügung stehen. Die Möglichkeiten werden kurz vorgestellt, sowie deren Zugangswege und Spezifikationen dargestellt.

\subsection{Anbieter und Werkzeuge}

\subsubsection{DNB}

Die Deutsche Nationalbibliothek ermöglicht seit 1913 einen Zugriff auf das kulturelle und wissenschaftliche Erbe Deutschlands und bietet mit einem vielfältigen Dienstleistungsangebot eine hohe Servicequalität. Das Gesetz über die Deutsche Nationalbibliothek ${ }^{164}$ sichert mit einem Pflichtexemplarrecht der DNB die Bestände, die in deutschen Verlagen erscheinen, zu. Durch die politische Teilung des Landes sind 2 Standorte entstanden. Deutschland hat daher seit der Wiedervereinigung des Landes eine doppelte Sicherung der Bestände, da ein Exemplar nach Leipzig und eines nach Frankfurt geht. Für die Musikbibliotheken relevante Bestände werden im deutschen Musikarchiv vorgehalten, das seit 2011 in einem Anbau den DNB Leipzig verortet ist. $^{165}$

Viele Angebote der DNB sind speziell für Bibliotheken zugeschnitten und über das Internet nutzbar. „Die Deutsche Nationalbibliothek bietet mit ihren Digitalen Diensten umfangreiche Dienstleistungen im Bereich der Datenübernahme (Export), der Kataloganreicherung, der dauerhaften Identifizierung von Online-Ressourcen und der Langzeitarchivierung an. “166 Mit einem neuen Geschäftsmodell ${ }^{167}$ stellt die DNB seit dem 1. Juli 2015 alle Titeldaten sowie die Normdaten der Gemeinsamen Normdatei (GND) kostenfrei unter einer "Creative Commons Zero" Lizenz (CCO 1.0) ${ }^{168}$ zur freien Nachnutzung zur Verfügung. In diesem Zusammenhang ist der Z39.50-Server abgeschaltet worden. Ein Datenbezug ist über SRU oder OAI möglich.

Für die Öffentlichen Bibliotheken sind die Bereiche Datenshop, Neuerscheinungsdienst, SRUSchnittstelle und die Kataloganreicherung mit Inhaltsverzeichnissen (TOC-Service) derzeit sinnvoll nutzbar. Die OAI-Schnittstelle sowie der Linked Data Service sind Themen, an die sich Öffentliche Bibliotheken auch herantasten sollten. Wünschenswert ist ein Einbinden der kostenfreien Angebote über Schnittstellen im jeweiligen Bibliotheksmanagementsystem. Die

\footnotetext{
164 Siehe: https://www.gesetze-im-internet.de/dnbg/BJNR133800006.html (02.02.2019).

165 Plassmann et al. 2011. S. 77.

166 Deutsche Nationalbibliothek o.J.a.

167 Deutsche Nationalbibliothek o.J.b.

${ }^{168} \mathrm{Vgl.} \mathrm{https://creativecommons.org/publicdomain/zero/1.0/deed.de} \mathrm{(25.02.2019).}$
} 
Ergebnisse der Expertenbefragung (siehe Anhang 3) zeigen jedoch, dass dies noch nicht in allen Systemen möglich ist.

Die Möglichkeit der Datennutzung der DNB besteht auch ohne eine SRU-Nutzung durch die direkte Downloadmöglichkeit im Datenshop. Der Import von Datendateien ist in allen 4 eingesetzten Bibliotheksmanagementsystemen gegeben. Zu kaufende oder zu katalogisierende Titel können direkt gesucht und bei Vorhandensein der Daten direkt über die Importfunktionen der Programme importiert werden. In der jeweiligen Bestell- oder Katalogisierungsmaske kann dann ergänzt oder auch gelöscht werden. Dieser Weg der Datenübernahme ist in einer Schrittfür-Schritt-Anleitung in Anhang 4 dargestellt.

Eine weitere Variante ist die eingesetzte Vorakzession ${ }^{169}$ in größeren Bibliotheken. Mögliche Kauftitel können von Fachangestellten oder sonstigem Personal vorbereitet werden, indem sie über den direkten Download in eine 2. Datenbank importiert werden. Bei Einbindung der 2. Datenbank als Fremddatenpool kann der Fachberater dann in einem direkten Vorgang die Titel über die jeweiligen Bestellmasken abrufen.

Die Frage $11^{170}$, ob die Bibliothek eine 2. Datenbank vorhält wurde von 13\% der Bibliotheken mit ,ja' beantwortet. Eine 2. Datenbank hat den Vorteil, dass darin erfasste Titel nicht im Produktivsystem und im OPAC sichtbar sind. Bei einem Import muss dadurch keine Kontrolle pro Einzeltitel erfolgen. Geprüft werden muss jedoch die generelle Anbindung der Datenfelder, da bei Übernahme ins Produktivsystem bei der Bestandsbestellung i.d.R. noch keine Prüfung des Katalogisats erfolgt. Diese Kontrolle findet erst nach Eingang des Mediums in der Katalogisierung statt. Durch den Import aus der 2. Datenbank übernommene Datenfelder können sowohl in der Katalogansicht als auch in der Statistik zu ungewollten Effekten führen. Diese Variante einer 2. Datenbank kann genutzt werden, um bei der ekz im Abonnement zum Informationsdienst bezogene Daten abzuspeichern, um sie dann direkt bei einer Bestellung importieren zu können.

\subsubsection{Bibliotheksverbünde}

Die Bibliotheksverbünde stellen zum Teil Ihre Daten zum Download oder zur Übernahme durch Schnittstellen zur Verfügung. Durch die regional eigenständigen Verbundkataloge sind die Datenbestände heterogen. Ein Zugriff auf mehrere Systeme ist möglich und in einigen Fällen auch vorteilhaft. Die meisten Kataloge sind im Netz frei verfügbar, können jedoch nicht von allen Bibliotheksmanagementsystemen abgefragt werden. Insofern ist die Bibliothek vom System abhängig, es sei denn, man nutzt die freien Schnittstellen außerhalb des eigenen Systems.

\footnotetext{
169 Bestellvorbereitung, wie z.B. Dublettenprüfung.

170 Frage 11: ,Betreiben Sie in Ihrer Bibliothek eine zweite Datenbank mit Katalogdaten?’
} 
Prinzipiell ist davon auszugehen, dass die gesuchten Bestandsdaten in der DNB verfügbar sind, da die Öffentlichen Bibliotheken vorwiegend deutschsprachige Medien einarbeiten. Eine Suche in weiteren Datenbanken bringt jedoch mitunter Vorteile bei der Titelaufnahme, da weitere Angaben in den Verbünden erfasst worden sind. Die Kategorien: Klassifikation oder Schlagworte sind in der Frage nach den bevorzugten Daten besonders häufig genannt. Diese Daten liefert die DNB jedoch nicht oder nicht kontinuierlich. Ebenfalls problematisch ist der Zeitpunkt der Erfassung. Öffentliche Bibliotheken haben den Anspruch auf das Vorhalten von aktuellsten Medien. Eine Titelaufnahme ist dadurch in der DNB häufig noch nicht erfolgt. Durch die direkte Katalogisierung in den Bibliotheksverbünden finden sich gerade diese Daten jedoch von anderen Bibliotheken.

Die folgende Übersicht stellt die Vorteile für Titelaufnahmen für Öffentliche Bibliotheken in Sachsen-Anhalt heraus.

Tab.: 7 Übersicht der Datenangebote der Verbünde

\begin{tabular}{|c|c|c|c|c|}
\hline Verbünde & Portal / Lizenz & Schnittstellen & $\begin{array}{l}\text { Vorteile für ÖB } \\
\text { Sachsen-Anhalt }\end{array}$ & $\begin{array}{l}\text { Direkter } \\
\text { Download } \\
\text { aus Portal } \\
\text { (MAB2 oder } \\
\text { MARC21) }\end{array}$ \\
\hline $\begin{array}{l}\text { BVB/ } \\
\text { KOBV }\end{array}$ & $\begin{array}{l}\text { http://www.gateway } \\
\text {-bayern.de/ } \\
\text { (CCO-Lizenz) }\end{array}$ & $\begin{array}{l}\text { Freie } \\
\text { Exportschnittstellen } \\
\text { Z39.50 und SRU }{ }^{171}\end{array}$ & $\begin{array}{l}\text { Großer } \\
\text { Bestands- } \\
\text { nachweis } \\
\text { (incl. KOBV- } \\
\text { Bibliotheken) }\end{array}$ & $\begin{array}{l}\text { Kein direkter } \\
\text { Export } \\
\text { möglich }\end{array}$ \\
\hline GBV & $\begin{array}{l}\text { https://gso.gbv.de/D } \\
\text { B=1.126 oder } \\
\text { https://gso.gbv.de/D } \\
\text { B=2.226/ } \\
\text { (CCO-Lizenz) }\end{array}$ & $\begin{array}{l}\text { Freie } \\
\text { Exportschnittstellen } \\
\mathrm{Z39.50} \text { und } \mathrm{SRU}^{172}\end{array}$ & $\begin{array}{l}\text { Andere } \\
\text { Bibliotheken mit } \\
\text { KAB- } \\
\text { Klassifikation }\end{array}$ & $\begin{array}{l}\text { Kein direkter } \\
\text { Export } \\
\text { möglich }\end{array}$ \\
\hline $\mathrm{hbz}$ & $\begin{array}{l}\text { https://lobid.org/ } \\
\text { (CCO-Lizenz) }\end{array}$ & $\begin{array}{l}\text { Exportschnittstellen } \\
\text { hbz Z39.50 }{ }^{173} \text { (nach } \\
\text { kostenfreier } \\
\text { Anmeldung) und SRU }\end{array}$ & & $\begin{array}{l}\text { direkter } \\
\text { Export in } \\
\text { MARCXML } \\
\text { möglich }\end{array}$ \\
\hline SWB & $\begin{array}{l}\frac{\text { http://swb.bsz- }}{\underline{\text { bw.de }}} \\
\text { (CcO-Lizenz) }\end{array}$ & $\begin{array}{l}\text { Exportschnittstellen } \\
\text { über } Z 39.50^{174} \text { und } \\
\mathrm{SRU}^{175}\end{array}$ & $\begin{array}{l}\text { Sächsische } \\
\text { Bibliotheken } \\
\text { sind enthalten - } \\
\text { dadurch KAB } \\
\text { Angaben und } \\
\text { ÖB-relevante } \\
\text { Schlagworte }\end{array}$ & $\begin{array}{l}\text { direkter } \\
\text { Export in } \\
\text { MARC21 und } \\
\text { MAB2 } \\
\text { möglich } \\
\text { (Format prüfen) }\end{array}$ \\
\hline
\end{tabular}

\footnotetext{
171 http://www.bib-bvb.de/open-data.html (02.02.2019).

172 Vgl. https://www.gbv.de/benutzer/z39.50-zugang/informationen-zum-z39.50-zugang-des-gbv (03.03.2019).

173 Hochschulbibliothekszentrum des Landes Nordrhein-Westfalen 2019.https://www.hbznrw.de/produkte/verbunddienstleistungen (03.03.2019).

${ }^{174}$ MAB2 Schnittstelle des Z39.50 Servers wird bis 31.12.2020 gepflegt.

175 https://wiki.bsz-bw.de/doku.php?id=v-team:daten:datendienste (03.03.2019).
} 
Möglichkeiten der kostenfreien Datenübernahme

\subsubsection{Zack}

URL: https://z3950.de/zack/

„Zack ist eine Suchmaschine für Bibliotheksdatenbanken, die über das Z39.50 Protokoll ansprechbar sind. Das Ergebnis sind strukturierte Daten (MAB2, MARC), die in das eigene Bibliothekssystem übernommen werden können. Bei der verteilten Suche wird gleichzeitig in mehreren Datenbanken gesucht. Dubletten werden als solche erkannt. ${ }^{1176}$

Das ZACK-Gateway kann über eine feldbasierte Konvertierung sowohl von MAB2 in MARC, als auch von MARC in MAB2 identische Felder übertragen. Es stellt für Öffentliche Bibliotheken, die keine Möglichkeit eines direkten Fremddatenimportes haben, seit vielen Jahren ein sehr gewinnbringendes Tool dar. So lange das Z39.50-Protokoll noch im Einsatz ist und abgefragt werden kann, kann die Datenübernahme über dieses Werkzeug im Browser jedes internetfähigen PCs durchgeführt werden.

Abgefragt werden können derzeit 49 Bibliotheken. Die DNB-Daten sind in MARC abrufbar.

KVK

http://kvk.bibliothek.kit.edu

Der KVK bietet als Metasuchmaschine eine Suche über mehrere Datenbanken an. Die Treffer leiten den Suchenden zum jeweiligen Portal, dessen Übernahmemodalitäten dann jeweils greifen.

176 https://www.z3950.de/zackSchneider 1999 (10.02.2019). 


\section{Fremddatenübernahme in den eingesetzten Bibliotheksmanagementsystemen}

Dieses Kapitel zeigt die Möglichkeiten der Fremddatenübernahme der in Sachsen-Anhalt eingesetzten 4 Bibliotheksmanagementsysteme anhand der in den Expertengesprächen ermittelten Grundvoraussetzungen auf. Bezug genommen wird auf die Umsetzung der Einführung von MARC21 und RDA.

\section{1.allegro-OEB - Büchereizentrale Niedersachsen}

allegro-OEB ist mit 14 Bibliotheken (35\%) das in den Öffentlichen Bibliotheken Sachsen-Anhalts am Häufigsten eingesetzte Bibliotheksmanagementsystem. Es wird in Bibliotheken der Gemeinden bis zu 40.000 Einwohnern verwendet. Die meisten Einsatzorte (50\% der allegroAnwender) finden sich in Gemeinden zwischen 10 und 20.000 Einwohnern.

allegro-OEB wurde mit Einführung vom MARC21 und RDA in Deutschland auf die neuen Anforderungen umgestellt. Es ist eine SRU-Schnittstelle implementiert, die für die 3 in SachsenAnhalt wichtigsten Datenbanken direkte Zugriffe gewährt: DNB, ÖVK des GBV und GVK des GBV. Die Software ermöglicht die Einbindung der GND-Daten und somit eine Möglichkeit, Metadaten normgerecht übernehmen zu können.

allegro-OEB stellt eine Anleitung zur Datenübernahme zur Verfügung, die sich im Anhang dieser Arbeit befindet. Im Rahmen eines Wartungsvertrages kann die Einrichtung der Fremddatenübernahme mit der Büchereizentrale gemeinsam vorgenommen werden. Liegt kein Wartungsvertrag vor, kann eine Einrichtung über Aufwandsberechnung erfolgen.

Mit diesen Angeboten sollten die allegro-OEB anwendenden Bibliotheken komfortabel eine Fremddatenübernahme durchführen, sowie Ihre Datenbestände darüber in RDA und MARC21 (Übernahme binär und in XML möglich) abbilden können.

Die Umfrage ergab jedoch ein anderes Bild. In allen Mediengruppen lag die Katalogisierung ,per Hand' bei über 50\%, die Übertragung findet bei 43\% noch über Z39.50 statt (dazu 29\%, die diese Frage nur mit ,nicht bekannt' beantworten konnten). 29\% der Bibliotheken katalogisieren noch in RAK-ÖB.

Bei der Verwendung des aktuellen Datenformates liegen die allegro-Anwender mit 58\% MARC21-Anwendern (8 Bibliotheken) im Vergleich zu den anderen Systemen sehr hoch.

$\rightarrow$ allegro-OEB.Dokumentation.Fremddatenübernahme - siehe Anhang 8

$\rightarrow$ Videoanleitung Fremddatenübernahme: https://www.youtube.com/watch?v=cxhnQbX5LCM 


\subsection{BIBLIOTHECAplus - OCLC GmbH}

Das Bibliotheksmanagementsystem BIBLIOTHECAplus wurde von 12 Einrichtungen (30\%) der teilnehmenden Bibliotheken als Bibliotheksmanagementsoftware angegeben.

Die Verteilung reicht von Bibliotheken in Gemeinden mit unter $10.000 \mathrm{zu}$ betreuenden Einwohnern bis zu den 3 Großstadtbibliotheken, die sich alle 3 für dieses System entschieden haben.

BIBLIOTHECAplus bietet für die Fremddatenübernahme eine ausgereifte Z39.50-Schnittstelle an, die sowohl MAB2-Daten als auch MARC21-Daten in die sogenannte ,alte Erfassungsmaske' importieren kann. MAB2-Daten können über die Zwischenablage auch per Kopieren und Einfügen erfasst werden und über einen Parameter komfortabel gefiltert werden. Dieser Weg kann mit dem ZACK-Gateway auch von Bibliotheken genutzt werden, die keinerlei Freischaltungen von Ports erhalten können (siehe Anleitung 5).

Die Änderung des Katalogisierungs-Regelwerkes führte in BIBLIOTHECAplus zur Erstellung einer ,neuen Erfassungsmaske'. In diese Maske können MARC21 Daten eingespielt werden. Hierbei ist die Erfassung jedoch noch nicht in allen Feldern und Ausprägungen von RDA möglich.

Das Datenangebot der ekz kann - bei Gewährleistung der entsprechenden Konditionen - über eine proprietäre ekz-Schnittstelle abgefragt werden. Ein Import der DNB und des GBV über die SRU-Schnittstellen ist derzeit noch nicht möglich. Weitere Fremddatenübernahmen sind geplant.

OCLC bietet bei bestehenden Wartungsverträgen Unterstützung der Fremddatenübernahme an .

$\rightarrow$ Optionen zur Fremddatenübernahme in BIBLIOTHECAplus - siehe Anhang Nr. 7

\subsection{LIBRARY for Windows - Fleischmann Software Vertriebs GmbH ${ }^{177}$}

Das Bibliotheksmanagementsystem LIBRARY for Windows wird von den abgefragten Bibliotheken sechs mal (15\%) in Gemeinden bis 30.000 zu betreuenden Einwohnern eingesetzt. Die Software unterstützt MAB2 für Datenimport und -export, sowie MARC21 (binär und XML) für den Import. Die Daten werden in der Datenbank in einem internen Format abgelegt. Für die Konvertierung wird die Konkordanzliste MAB2 auf MARC21 $1^{178}$ eingesetzt. Fremddaten können in der Katalogisierung über die ISBN mittels Z39.50 direkt importiert werden. Weiterhin ist es möglich, über den ZACK-Client Daten mehrerer Titel in eine Datei abzuspeichern und diese dann in die Datenbank zu importieren. Auf diesem Weg können ebenfalls über eine SRU-Schnittstelle abgerufene Daten importiert werden. 
Fremddatenübernahme in den eingesetzten Bibliotheksmanagementsystemen

Der Fremddatenimport wird in Anwenderschulungen gelehrt und die zu importierenden Kategorien werden zentral festgelegt. Eine direkte Filterung der Daten kann nicht vorgenommen werden. Durch den möglichen Import von RDA-Daten können die Datenbestände nach und nach in das neue Katalogisierungformat überführt werden. Support und Wartung besteht bei Einsatz des Importmoduls - auch bei Änderungen z.B. der Kategorien.

Die Übernahme erfolgt bei den teilnehmenden Bibliotheken jedoch in fast allen Mediengruppen im Bereich zwischen 76 und $100 \%$ als manuelle Katalogisierung. Nur eine Bibliothek gibt an, dass kostenfreie Fremddaten genutzt werden. Die Katalogisierung findet in RAK bzw. in einem RAK/RDA-Mix statt, GND-Daten werden nicht genutzt. Die Daten werden in MAB2 aufgenommen. In 2 Bibliotheken wird LIBRARY for Windows im Hosting des Systemanbieters eingesetzt. Die Bibliotheken verlagern somit die Software und Datenhaltung, Updates und Wartung - aber auch große Bereiche des Datenschutzes in die Softwarefirma. Das Bibliotheksmanagementsystem wird über eine Citrix-Anwendung in den Bibliotheken genutzt. Für die Fremddatenübernahme bringt dies den Vorteil der komfortablen Freischaltung von Ports und vorhandenen Zugängen zu Datenbanken.

\subsection{WinBIAP - datronic IT-Systeme GmbH \& Co. KG}

Die Software WinBIAP ist eine Software, die sich unter anderem auch an kleinere und mittelstädtische Bibliotheken richtet und diesbezüglich versucht, die Spezifika dieser Einrichtungen abzubilden. Sie wird in der Umfrage fünf mal (13\%) als eingesetztes Bibliotheksmanagementsystem angegeben. WinBIAP ist in der Version 5.0 an die neuen Formate und Protokolle angepasst worden. Diese Version ist noch nicht in allen Bibliotheken im Einsatz, die Informationen beziehen sich jedoch vorwiegend auf die vorliegende Version 5.0.

WinBIAP kann MAB2 und MARC21 (binär und XML) verarbeiten, legt die Metadaten in der Datenbank ebenfalls in einem Internformat ab. In diesem Format werden nicht genutzte Felder der Datenformate für eigene Informationen, wie Klarschriftsystematik oder Mediengruppen, umgewidmet. Ein für alle kostenlos integrierter Z39.50-Client macht die Übernahme bisher direkt in der Erfassungsmaske möglich - über SRU in der Version 5.0 ebenfalls im Format MARC21. In der jeweiligen Ausprägung ist die Übernahme bei MAB2 an die ekz und über MARC21 an die DNB angelehnt. Die Bibliothek kann bei der Übernahme Kategorien filtern. 
Fremddatenübernahme in den eingesetzten Bibliotheksmanagementsystemen

Übernommen werden von den WinBIAP-Bibliotheken prozentual die meisten Mediengruppen in den unteren Prozentbereichen, was auf eine einfach nutzbare Fremddatenübernahme schließen lässt.

Mit dem Umstieg auf die Version 5.0 werden alle Daten der Bibliotheken automatisch in MARC21 konvertiert und an RDA angepasst.

Unterstützung finden die Bibliotheken durch telefonischen Support sowie durch Fernwartung. Bibliotheken können auf ein gehostetes System umsteigen. Die WinBIAP Cloud wird als Software as a Service (SaaS) angeboten. Die Server stehen in Deutschland, weshalb der Datenschutz voll gewährleistet ist. Mit dem Umstieg in die Cloud wird den Bibliotheken automatisch ein WebOPAC zur Verfügung gestellt. Die Fremddatenübernahme ist im Hosting deutlich vereinfacht, da sich die Softwarefirma um freizuschaltende Ports und Zugriffe auf Datenbanken kümmert.

Datronic arbeitet mit dem Buchhandel zusammen - so können vor allem kleine Bibliotheken über die Buchhandelsschnittstelle auf VLB ${ }^{179}$-Daten zugreifen und haben schon vor der Veröffentlichung der Medien die Metadaten zur Bestellung verfügbar.

${ }^{179}$ Siehe: https://vlb.de/ (12.03.2019). 


\section{Cloudbasierte Angebote und mögliche Zukunft der Katalogisierung}

Liegt die Zukunft von Bibliotheksmanagementsystemen in der Cloud?

Die Frage, ob in den teilnehmenden Bibliotheken konkrete Überlegungen zum Wechsel in ein Cloud-System bestehen, wurde in Frage $7^{180}$ gestellt.

3 von 39 Bibliotheken und somit 8\% planen in den nächsten 5 Jahren auf ein cloudbasiertes System umzustellen. Durch verbale Ergänzung wurde deutlich, dass mehrfach nicht der nicht vorhandene Wunsch, sondern der mangelnde Etat der Grund ist, dass ein Wechsel bisher nicht geplant ist.

Neben der Variante des Hostings des bestehenden Bibliotheksmanagementsystems wächst die Zahl der Anbieter, die cloudbasierte Systeme auch für Öffentliche Bibliotheken anbieten, z.B.:

- LMS Cloud ${ }^{181}$ - ein Angebot, das auf der Open-source-Software Koha basiert. Koha wird von einer Community betreut. Die Firma LMS-Cloud bietet für die vorhandene Grundversion von Koha weitere Schnittstellen an.

- WMS $^{182}$ (WorldShare Management Services) - cloudbasierte Plattform auf Basis des Worldcat

- Quria ${ }^{183}$ der Firma Axxiel, eine Cloud-basierte, "digital-first Library Services Platform"

\section{Linked Open Data}

Linked-Data-Initiativen werden seit ca. 2011 von wichtigen Institutionen wie der Library of Congress, OCLC oder der DNB durchgeführt. Wieso sollte hier die Zukunft der Bibliotheksdaten liegen?

Der wichtigste Grund ist wohl die Sichtbarkeit der Daten im Web. Bibliothekskataloge sind häufig noch Teil des „Deep Web“. Ein Nutzer, der in einer gängigen Suchmaschine nach einem Thema sucht, bekommt daher in den seltensten Fällen die passenden Medien einer Bibliothek angezeigt. Eine Anzeige der Medien, geografisch zugeordnet in der für ihn am nächstgelegenen Bibliothek. ${ }^{184}$ - das ist jedoch das Ziel, das Bibliotheken mit der Art ihrer Titelaufnahmen verfolgen sollten. Linked Open Data bietet einen Weg dahin, indem es dafür sorgt, dass die bibliographischen Entitäten eindeutig verlinkbar sind. ${ }^{185}$

\footnotetext{
${ }^{180}$ Frage 7: ,Planen Sie als Bibliothek in nächster Zukunft ein cloudbasiertes Bibliotheksmanagementsystem einzusetzen?"

${ }^{181}$ Siehe: LMSCloud GmbH o.J. https://www.Imscloud.de/ (09.03.2019).

182 Siehe: OCLC 2019b. https://www.oclc.org/de/worldshare-management-services.html (13.03.2019).

183 Siehe: Axiell Quria o.J.- für eine neue Generation von Bibliotheken (09.03.2019).

${ }^{184}$ Wie z.B. in https://www.bibliotheken.at/region.

185 Fürste 2011, S. 76.
} 


\section{Fazit}

Öffentliche Bibliotheken sind eigenständige kommunale Einrichtungen, die von der Fachstelle für Öffentliche Bibliotheken beratend betreut werden und denen das Land über Förderungen Unterstützung gewährt. „Werden sie mit einem klaren Auftrag, fördernden Rahmenbedingungen und ausreichenden Mitteln versehen, können sie ihren Beitrag zur erforderlichen leistungsorientierten Bildungsinfrastruktur vervielfachen. ${ }^{186}$

Die Studie hat versucht, die Frage zu beantworten, ob Öffentliche Bibliotheken im Bundesland Sachsen-Anhalt die derzeitigen technischen Möglichkeiten nutzen, ihre Katalogisate durch Fremddaten zu beziehen oder anzureichern, um den Bibliotheksnutzern somit inhaltlich aufbereitete Daten in einer vertretbaren Zeit bereitstellen zu können.

Um dies zu realisieren, wurden eine Online-Umfrage bei allen hauptamtlich geleiteten Öffentlichen Bibliotheken und Expertengespräche mit den Systemanbietern der Bibliotheksmanagementsysteme durchgeführt. So kann ein realistisches Bild der Fremddatennutzung in der Katalogisierungspraxis gezeichnet werden.

Der Bereich der Katalogisierung einer Öffentlichen Bibliothek liegt aufgrund fehlender kooperativer Zusammenarbeit in der vollen Verantwortung jeder einzelnen Bibliothek.

Die Ergebnisse zeigen, dass die derzeitige Katalogisierungssituation in Sachsen-Anhalts Öffentlichen Bibliotheken nicht dem derzeit angestrebten Standard ${ }^{187}$ entspricht. Mit $46 \%$ nutzen 18 Bibliotheken keine Fremddaten. Ursachen sind mangelnde Technik, fehlendes Knowhow aber auch fehlende Zeit und/oder Bereitschaft, neue Wege auszuprobieren. Hier besteht Weiterbildungsbedarf. Die Umstellung auf RDA und MARC21 hat bisher in Sachsen-Anhalts Öffentlichen Bibliotheken nur in wenigen Einrichtungen stattgefunden.

Eine Problematik in der Bewältigung und Aufarbeitung der Thematik dieser Arbeit besteht in der Vermittlung der bestehenden Anforderungen zwischen der Softwarefirma des Bibliotheksmanagementsystems, der Bibliothek und der betreuenden IT-Firma. Dazu ist in erster Linie die Bereitschaft des Bibliothekspersonals notwendig, sich den neuen Themen der Katalogisierung zu stellen. Weiterhin muss konkretes Wissen vorliegen, welche technischen Voraussetzungen in der Bibliothek gegeben sein müssen, um eine Fremddatenübernahme umsetzen zu können. Die Möglichkeiten eines Hostings oder der Miete des Bibliotheksmanagementsystems werden zunehmend genutzt und bieten gerade für Bibliotheken mit Problemen der Infrastruktur neue Chancen, Ihren Nutzern ein besseres Angebot bieten zu können.

\footnotetext{
186 Beger 2004, S. 11.

${ }^{187}$ Z.B. bundesweite Vorgaben zum Einsatz von RDA und MARC21.
} 
Katalogisierung hat das Ziel, Medien zu erfassen, aber auch ihr Vorhandensein darzustellen und zu verorten. Der Bibliotheksnutzer kann dies über einen OPAC- oder Discoverysystem recherchieren. Liegt eine solche Möglichkeit in kleinen Bibliotheken nicht vor, sollte eine landesweite Darstellung im Rahmen des ÖVK oder in den schon vorhandenen Angeboten ${ }^{188}$ geprüft werden.

Die technische Umstrukturierung des ÖVK ab März 2019 bietet die Gelegenheit, die Fremddatennutzung für die teilnehmenden Bundesländer neu zu überdenken und zu organisieren. Wenn eine automatisierte Datenübertragung in kürzeren Intervallen möglich ist, ist der ÖVK für eine Datenübernahme aller teilnehmenden Bibliotheken prädestiniert. Ein regelmäßiger Import der Metadaten der großen Öffentlichen Bibliotheken der 3 Bundesländer bringt einen Großteil der benötigten nachnutzbaren Titelaufnahmen auch für alle kleineren Bibliotheken in die Datenbank.

Weitere Möglichkeiten der kostenfreien Datenübernahme bestehen seit 2015 durch das lizenzfreie Vorhalten der Titeldaten der DNB und einiger Bibliotheksverbünde, sowie weiterhin durch die einfache browserbasierte Übernahmemöglichkeit über das ZACK-Gateway. Zunehmend tritt der Buchhandel als Partner für Bibliotheken auf, nicht nur traditionell durch Medienangebote, sondern auch mit dem Angebot von Metadaten oder Medienbearbeitung.

Nicht zuletzt ist der Kauf im regionalen Buchhandel eine Möglichkeit jeder Bibliothek, Umsatzsteuern in der jeweiligen Region zu belassen und durch kurze Transportwege und wiederverwendbare Transportkisten eine nicht unerhebliche ökologische Last durch überregionale Pakete mit allen Auswirkungen auf Luftbelastung und Papiermüll zu begrenzen.

In dieser Arbeit wurden die zur Verfügung stehenden Materialien für die 4 verschiedenen im Einsatz befindlichen Bibliotheksmanagementsysteme zusammengefasst bzw. erstellt. Notwendig wird jedoch eine Institution sein, die den Bibliotheken konkret Hilfestellung gibt. Diese Anforderung könnte durch ein Projekt der Fachstelle oder/und des GBV im Rahmen der Förderrichtlinien des Bundeslandes umgesetzt werden. Die Schlussfolgerungen im Ergebnis dieser Arbeit werden in einem Konzeptvorschlag im Anhang 6 zusammengefasst.

Mit der Neustrukturierung des ÖVK sollten weitere Möglichkeiten wie eine Nutzung der GNDDaten und des Neuerwerbungsdienstes des Buchhandels aber auch die angebotene Möglichkeit der Datenübernahme und/oder der Katalogisierung im Worldcat geprüft werden.

188 Neben dem BRISEOpac ist der BVS eOPAC der Firma IBTC im Einsatz siehe: http://www.ibtc.de/bvs-eopac-3.html. 
Anleitungen / Leitfäden

\section{Anleitungen / Leitfäden}

Im Anhang dieser Arbeit werden konkrete Handreichungen und Empfehlungen abgelegt, um einen direkten Nutzen für die Bibliotheken anzubieten. Weiterhin findet sich dort ein Konzeptvorschlag für ein Katalogisierungsprojekt für das Land Sachsen-Anhalt.

- Anleitung Datenübernahme aus dem Datenshop der DNB - Anhang 4

- Anleitung Datenübernahme mit dem ZACK-Gateway - Anhang 5

- Konzeptvorschlag Katalogisierungsprojekt - Anhang 6

- Optionen zur Fremddatenübernahme in BIBLIOTHECAplus (Originalmaterial OCLC) Anhang 7

- allegro-OEB Anleitung.Dokumentation.Fremddatenübernahme (Originalmaterial der Büchereizentrale Niedersachsen ) - Anhang 8 


\section{Literaturverzeichnis}

amazon.de [o.J.].

https://www.amazon.de/ (01.03.2019).

Axiell Quria: für eine neue Generation von Bibliotheken [o.J.]. Lund. https://www.axiell.de/quria/ (09.03.2019).

Baum, Heidemarie [1992]: Fremddatenübernahme bei EDV-Anwendungen in Öffentlichen Bibliotheken.

In: Bibliothek Forschung und Praxis 16 (1992), Nr. 3, S. 376-418.

Beger, Gabriele (Hg.) [2004]: Bibliothek 2007: Strategiekonzept. Bertelsmann-Stiftung; Bundesvereinigung Deutscher Bibliotheksverbände. 3. Aufl. Gütersloh: Verl. BertelsmannStiftung, 2004.

Bibliographic Framework Initiative (BIBFRAME). [0.J.]. http://bibframe.org/ (07.02.2019).

Bibliotheken '93. Strukturen, Aufgaben, Positionen (1994). Berlin und Göttingen: Bundesvereinigung Deutscher Bibliotheksverbände. [1994]. http://www.bibliotheksportal.de/fileadmin/user_upload/content/bibliotheken/strategie/ dateien/bibliotheken93.pdf (31.12.2018).

Bibliotheksplan 1973: Entwurf eines umfassenden Bibliotheksnetzes für die Bundesrepublik Deutschland / Deutsche Bibliothekskonferenz [1973]. Berlin. https://bibliotheksportal.de/content/uploads/2017/10/bibliotheksplan1973.pdf.

Bibliotheksservice-Zentrum Baden-Württemberg [2017]: Datendienste. Konstanz, 2017. https://wiki.bsz-bw.de/doku.php?id=v-team:daten:datendienste (03.03.2019).

BibliotheksVerbund Bayern [2016]: B3Kat - Open Data, 2016. http://www.bib-bvb.de/open-data.html (02.02.2019).

Buch und Kunst: Bibliotheksservice [o.J.]. http://www.buk-stender.de/index.php (01.03.2019).

Büchereizentrale Niedersachsen [2019]: allegro-OEB. Lüneburg, 2019. https://www.bz-niedersachsen.de/allegro-oeb.html (13.03.2019).

Bundesministerium der Justiz und für Verbraucherschutz [2006]: Gesetz über die Deutsche Nationalbibliothek (DNBG), 2006.

https://www.gesetze-im-internet.de/dnbg/BJNR133800006.html (02.02.2019).

Bund-Länder-Arbeitsgruppe Bibliothekswesen [1991]: Empfehlungen zur Förderung der Bibliotheken in den neuen Bundesländern. Berlin: Deutsches Bibliotheksinstiut, 1991 (DBIMaterialien / Deutsches Bibliotheksinstitut (Berlin), 106).

Conradt, Volker; Lohrum, Stefan [2017]: Gemeinsamer Verbünde-Index, gemeinsamer Fernleihindex: Ein Projekt der AG-Verbundsysteme. 106. Deutscher Bibliothekartag. BSZ; KOBV. Frankfurt am Main, 30.05.2017. https://opus4.kobv.de/opus4-bib-info/frontdoor/index/index/docld/2919 (11.03.2019).

Creative Commons [o.J.]: CCO 1.0 Universell (CCO 1.0): Public Domain Dedication, o.J. https://creativecommons.org/publicdomain/zero/1.0/deed.de (25.02.2019).

datronic IT-Systeme GmbH \& Co. KG [o.J.]: WinBIAP: Bibliothekssoftware. Augsburg, o.J. http://www.datronic.de/Bibliothekssysteme/WinBIAP (13.03.2019).

Deutsche Forschungsgemeinschaft und Wissenschaftsrat [2011a]: Gemeinsame Erklärung: Zur Zukunft der Bibliotheksverbünde als Teil einer überregionalen Informationsinfrastruktur in Deutschland. Köln, Bonn, 2011. https://www.wissenschaftsrat.de/download/archiv/1003-11.pdf (15.01.2019). 
Deutsche Forschungsgemeinschaft und Wissenschaftsrat [2011b]: Positionspapier zur

Weiterentwicklung der Bibliotheksverbünde als Teil einer überregionalen

Informationsinfrastruktur. Ausschuss für wissenschaftliche Bibliotheken und Informationssysteme. Bonn, 2011.

http://www.dfg.de/download/pdf/foerderung/programme/lis/positionspapier_ bibliotheksverbuende.pdf (15.01.2019).

Deutsche Nationalbibliothek [o.J.a]: Digitale Dienste, o.J. https://www.dnb.de/DE/Service/DigitaleDienste/digitaledienste_node.html (02.02.2019).

Deutsche Nationalbibliothek [o.J.b]: Geschäftsmodell für die Nutzung von Datendiensten, o.J. https://www.dnb.de/DE/Service/DigitaleDienste/Geschaeftsmodell/geschaeftsmodell_ node.html (02.02.2019).

Deutsche Nationalbibliothek [2008]: Konkordanz MAB2 - MARC21: Teil 1: Konkordanz MAB-TITEL MARC Bibliographic, 2008. https://d-nb.info/988382318/34 (26.02.2019).

Deutsche Nationalbibliothek [2016]: Formate und Schnittstellen/ MAB, 2016. https://www.dnb.de/DE/Standardisierung/Formate/MAB/mab_node.html (07.02.2019).

Deutsche Nationalbibliothek [2017]: Arbeitsgemeinschaft der Verbundsysteme, 2017. https://www.dnb.de/DE/Wir/Kooperation/AGVerbundsysteme/agverbund_node.html (19.02.2019).

Deutsche Nationalbibliothek [2018a]: SRU-Schnittstelle, 2018. http://www.dnb.de/DE/Service/DigitaleDienste/SRU/sru_node.html (25.02.2019).

Deutsche Nationalbibliothek [2018b]: RDA-Info: Schulungen. Deutsche Nationalbibliothek, 2018. https://wiki.dnb.de/display/RDAINFO/Schulungen (27.02.2019).

Deutsche Nationalbibliothek [2019a]: Informationsseite zur GND, 2019. https://wiki.dnb.de/display/ILTIS/Informationsseite+zur+GND (25.02.2019).

Deutsche Nationalbibliothek [2019b]: Neuerscheinungsdienst. Frankfurt am Main, Leipzig, 2019. https://www.dnb.de/DE/Service/DigitaleDienste/ND/nd.html (12.03.2019).

Diedrichs, Reiner; Goebel, Ralf [2018]: Veränderungen sind nötig. Kooperation des Bibliotheksservice-Zentrums Baden-Württemberg (BSZ) und der Verbundzentrale des GBV (VZG).

In: Achim Bonte u. Juliane Rehnolt (Hg.): Kooperative Informationsinfrastrukturen als Chance und Herausforderung. Berlin, Boston: De Gruyter, 2018.

ekz bibliotheksservice [2019a]: ekz - das Entdeckerteam für starke Bibliotheken. Reutlingen, 2019. https://www.ekz.de/ekz-bibliothekseinrichtung-ausstattung-service-und-medien/ (08.03.2019).

ekz bibliotheksservice [2019b]: Lektoratsdienste: Preise 2019, 2019. https://www.ekz.de/fileadmin/ekz-media/downloads/downloads_medien/ Bibliothekarische\%20Dienste/Lektoratsdienste_2019_Preisblatt.pdf (24.02.2019).

Fleischmann Software Vertriebs GmbH [2019]: Fleischmann Software Vertriebs GmbH. Leingarten, 2019.

https://www.fleischmann.org/ (13.03.2019).

Fürste, Fabian M. [2011]: Linked Open Library Data: Bibliographische Daten und ihre Zugänglichkeit im Web der Daten ; Innovationspreis 2011. Zugl.: Berlin, Humboldt-Univ., Magisterarbeit, 2010. Wiesbaden: Dinges \& Frick, 2011 (BIT online Innovativ, 33).

Gantert, Klaus [2016]: Bibliothekarisches Grundwissen. 9., vollständig aktualisierte und erweiterte Auflage. Berlin, Boston: de Gruyter Saur, 2016. http://dx.doi.org/10.1515/9783110321500.

Göttker, Susanne; Wein, Franziska (Hg.) [2014]: Neue Formen der Erwerbung. Berlin: de Gruyter Saur, 2014 (Bibliotheks- und Informationspraxis, 47).

Haller, Klaus; Fabian, Claudia [2004]: 10 Bestandserschließung. In: Rudolf Frankenberger (Hg.): Die moderne Bibliothek. Unter Mitarbeit von Rudolf Frankenberger. München: Saur, 2004, S. 222-261. 
Herrmann [und] Pflug GbR: Systembetreuung Softwareentwicklung Internetportal. http://www.herrmann-pflug.de/html/brise.htm (08.01.2019).

Hochschulbibliothekszentrum des Landes Nordrhein-Westfalen [o.J.]: lobid-gnd: Rechercheoberfläche \& LOD-API für die GND. Köln, o.J. https://lobid.org/gnd (13.03.2019).

Hochschulbibliothekszentrum des Landes Nordrhein-Westfalen [2018]: Bibliotheksstatistik. Hochschulbibliothekszentrum des Landes Nordrhein-Westfalen, 2018. https://www.hbz-nrw.de/produkte/bibliotheksstatistik (24.02.2019).

Hochschulbibliothekszentrum des Landes Nordrhein-Westfalen [2019]: Verbunddienstleistungen. Köln, 2019.

https://www.hbz-nrw.de/produkte/verbunddienstleistungen (03.03.2019).

Hohoff, Ulrich [2015]: Strukturen und Entwicklungen des wissenschaftlichen Bibliothekssektors in Deutschland: Die Bibliotheksverbünde.

In: Rolf Griebel, Hildegard Schäffler et al. (Hg.): Praxishandbuch Bibliotheksmanagement. Berlin, München, Boston: de Gruyter Saur, 2015 (Reference), S. 70-107.

Hugendubel Fachinformation [2018]: Bibliotheken, 2018. https://www.hugendubel.info/services/bibliotheken (01.03.2019).

IFLA [2001]: Die Dienstleistungen der Öffentlichen Bibliothek: IFLA/UNESCO Richtlinien für die Weiterentwicklung: Aufgestellt von einer Arbeitsgruppe unter dem Vorsitz von Philip Gill im Auftrag der Sektion Öffentliche Bibliotheken, 2001. https://www.ifla.org/files/assets/hq/publications/archive/the-public-library-service/pg01g.pdf.

Kemner-Heek, Kirstin [2012]: Konzeption und Angebot zukünftiger Bibliotheksmanagementsysteme: Bestandsaufnahme und Analyse. In: Kölner Arbeitspapiere zur Bibliotheks- und Informationswissenschaft (2012), Nr. 64. urn://nbn:de:hbz:79pbc-20120713580.

Kiefer, Andrea (31.01.2019a): Expertengespräch zu LIBRARY for Windows - Fleischmann. Interview mit xxxx. mp3-Datei.

Kiefer, Andrea (31.01.2019b): Expertengespräch zu WinBIAP. Interview mit Siegfried Fendt. mp3File.

Kiefer, Andrea (01.02.2019a): Expertengespräch zu allegro-OEB. Interview mit Joachim Oeding. mp3-File.

Kiefer, Andrea (01.02.2019b): Expertengespräch zu BIBLIOTHECAplus / OCLC. Interview mit Jana Heintel. mp3-File.

Kratzer, Mathias; Thiessen, Peter; Lohrum, Stefan et al. [2016]: Zum Stand des Projektes „Cloudbasierte Infrastruktur für Bibliotheksdaten“ (CIB). In: ZfBB 63 (2016), Nr. 5/6, S. 250-257. http://dx.doi.org/10.3196/1864295016635627.

Landesverwaltungsamt Sachsen-Anhalt [Erl. der StK vom 2017]: Richtlinie über die Gewährung von Zuwendungen zur Förderung von kulturellen und künstlerischen Projekten und kulturellen Institutionen: Kulturförderrichtlinie Sachsen-Anhalt, Erl. der StK vom 2017 (StK-6-57001). http://www.foerderdatenbank.de/Foerder-DB/Navigation/Foerderrecherche/suche.html? get=056c1600964814c7ac8c99bf030cba03;views; document\&doc=10722\&typ=RL (30.12.18).

Landesverwaltungsamt Sachsen-Anhalt [2019]: Kommunale hauptamtliche öffentliche Bibliotheken in Sachsen-Anhalt, 2019.

https://lvwa.sachsen-anhalt.de/das-lvwa/kultur-denkmalschutz/landesfachstelle-fueroeffentliche-bibliotheken/bibliotheken-in-sachsen-anhalt/ (03.03.2019).

Lange, Matthias [2018]: Verbundkatalog Öffentlicher Bibliotheken Worldcat für Öffentliche Bibliotheken. 22. Verbundkonferenz des GBV. GBV. Kiel, 29.08.2018. https://verbundkonferenz.gbv.de/wp-content/uploads/2018/09/lange_180829_oevk.pdf (13.03.2019). 
Library of Congress [1995]: ATTRIBUTE SET BIB-1 (Z39.50-1995):: SEMANTICS, 1995.

https://www.loc.gov/z3950/agency/bib1.html (09.03.2019).

Library of Congress [2016]: SRU: Search/Retrieval via URL, 2016. http://www.loc.gov/standards/sru/ (25.02.2019).

LMSCloud GmbH [o.J.]: LMSCloud: Das Bibliothekssystem mit dem Sicherheitsfaktor. München, o.J. https://www.Imscloud.de/ (09.03.2019).

Ministerium für Landesentwicklung und Verkehr des Landes Sachsen-Anhalt (Hg.) [2011]: Verordnung über den Landesentwicklungsplan 2010 des Landes Sachsen-Anhalt: Anhang 1, 2011.

http://www.landesrecht.sachsen-anhalt.de/jportal/docs/anlage/lr/st/a4f7f9e3-645f-4cf39543-bc9049df48b6-st230.10+2011+160+p+anhang_1.pdf (03.03.2019).

Münch, Vera [2014]: „Wir brauchen extrem viel Fort- und Weiterbildung“" In: b.i.t.online 17 (2014), Nr. 1, S. 50-54. https://b-i-t-online.de/heft/2014-01-index.php (01.03.2019).

mvb GmbH [2019]: vlb. Frankfurt am Main, 2019. https://vlb.de/ (12.03.2019).

Naumann, Ulrich; Umlauf, Konrad: Personalbedarf: Methoden der quantitativen PersonalbedarfsErmittlung: Abschnitt 4.3.2.

In: Hans-Christoph Hobohm (Hg.): Erfolgreiches Management von Bibliotheken und Informationseinrichtungen, Bd. 2. Nummer 62 - September 2018. 3 Bände. Hamburg: Dashöfer, S. 1-10. https://www.dasbibliothekswissen.de/Personalbedarf\%3A-Beispiel-f\%C3\%BCr\%C3\%96ffentliche-Bibliotheken.html.

Nolte, Adrian [1988]: Fremddatenübernahme in Öffentlichen Bibliotheken. In: Buch und Bibliothek 40 (1988), S. 151-160.

OCLC [2019a]: BIBLIOTHECAplus: Software für Bibliotheken, die begeistern. Oberhaching, 2019. https://www.oclc.org/de/bibliotheca.html (13.03.2019).

OCLC [2019b]: WorldShare Management Services: Eine integrierte Suite Cloud-basierter Anwendungen zur Bibliotheksverwaltung. Oberhaching, 2019. https://www.oclc.org/de/worldshare-management-services.html (13.03.2019).

Öffentliche Bibliothek (1973): Gutachten der Kommunalen Gemeinschaftsstelle für Verwaltungsvereinfachung (KGSt). Berlin: Deutscher Bibliotheksverband, Arbeitsstelle für das Bibliothekswesen. (Schriftenreihe Bildungsplanung. 2).

Oldenburg, Ray [1989]: The great good place: Cafés, coffee shops, community centers, beauty parlors, general stores, bars, hangouts, and how they get you through the day. 1st ed. New York: Paragon House, 1989.

Plassmann, Engelbert; Rösch, Hermann; Seefeldt, Jürgen et al. [2011]: Bibliotheken und Informationsgesellschaft in Deutschland: Eine Einführung. 2., gründlich überarbeitete und erweiterte Auflage. Wiesbaden: Harrassowitz Verlag, 2011.

Pohl, Adrian; Danowski, Patrick [2013]: (Open) Linked Data in Bibliotheken. s.l.: De Gruyter, 2013. http://www.doabooks.org/doab?func=fulltext\&rid=18046.

Röschlau, Edith [2017]: Bericht aus der 71. Sitzung der Arbeitsgemeinschaft der Verbundsysteme am 15. bis zum 17. November 2016 in Berlin.

In: Bibliotheksdienst 51 (2017), Nr. 5, S. 411-417. http://dx.doi.org/10.1515/bd-2017-0041 (14.02.2019).

Schade, Frauke [2016]: Praxishandbuch Digitale Bibliotheksdienstleistungen: Strategie und Technik der Markenkommunikation. Berlin/Boston: De Gruyter, 2016 (De Gruyter Reference). https://ebookcentral.proquest.com/lib/gbv/detail.action?doclD=4768872.

Schleihagen, Barbara [2018]: Wie gewinnen wir neues Personal für veränderte Bibliotheken?: die BID AG Personalgewinnung hat ihre Arbeit aufgenommen. In: BuB 70 (2018), Nr. 08-09, S. 470-473. https://www.b-u-b.de/wp-content/uploads/2018-08-1.pdf (06.02.2019). 
Schneider, W. [1999]: Zack-Gateway, 1999. www.z3950.de/zack (10.02.2019).

SpielTruhe: Mit Sicherheit die besten Spiele. https://www.spieltruhe.de/b2c_index.php (01.03.2019).

Statistisches Landesamt Sachsen-Anhalt (Hg.) [2018]: Statistischer Bericht: Bevölkerung und Erwerbstätigkeit, 2018.

https://statistik.sachsen-anhalt.de/fileadmin/Bibliothek/Landesaemter/StaLa/startseite/ Themen/Bevoelkerung/Berichte/Bevoelkerungsstand/6A102_02_17-A.pdf.

Umlauf, Konrad [2015]: Grundkurs Informationsaufbereitung: Vorlesungsskript. Institut für Bibliotheks- und Informationswissenschaft, 2015 (Berliner Handreichungen zur Bibliotheksund Informationswissenschaft, 189).

http://www.ib.hu-berlin.de/\%7Ekumlau/handreichungen/h189/.

Umlauf, Konrad (Hg.) [2016]: Grundwissen: Medien, Information, Bibliothek. Stuttgart: Anton Hiersemann Verlag, 2016 (Bibliothek des Buchwesens, Band 25).

VERBI GmbH [2019]: MaxQda. Codieren. Transkribieren. Analysieren. Präsentieren., 2019. https://www.maxqda.de/ (27.02.2019).

Verbundzentrale des GBV [o.J.a]: Bearbeitungsrichtilinie: Richtlinie zur Bearbeitung von Titelaufnahmen im GBVKat mit Anhängen. Göttingen, o.J. https://www.gbv.de/bibliotheken/verbundbibliotheken/02Verbund/01Erschliessung/ 02Richtlinien/03Bearbeitungsrichtlinie/index (26.02.2019).

Verbundzentrale des GBV [o.J.b]: Richtlinien zur Katalogisierung im GBVKat, o.J. https://www.gbv.de/bibliotheken/verbundbibliotheken/02Verbund/01Erschliessung/ 02Richtlinien (29.12.2018).

Verbundzentrale des GBV [o.J.c]: Stadtbibliothek Bremen: Die Stadtbibliothek Bremen katalogisiert im GVK. Göttingen, o.J. https://www.gbv.de/news/neues-aus-der-verbundzentrale/2013/stadtbibliothek-bremen.

Verbundzentrale des GBV [o.J.d]: Verbundzentrale des GBV (VZG), o.J. https://www.gbv.de/Verbund (10.02.2019).

Verbundzentrale des GBV [1996]: Verwaltungsabkommen über die Errichtung eines Bibliotheksverbundes (1996), 1996. https://www.gbv.de/Verbund/02GBV_1200.

Verbundzentrale des GBV [2019]: Verbundkatalog Öffentlicher Bibliotheken, 2019. https://gso.gbv.de/ (10.03.2019).

Wiesenmüller, Heidrun [2017]: Basiswissen RDA: Eine Einführung für deutschsprachige Anwender. 2. Aufl. Berlin, Boston: de Gruyter Saur, 2017.

Woppowa, Belinda [2018]: Katalogisierungspraxis an Öffentlichen Bibliotheken in Deutschland: Eine Analyse der aktuellen Situation. Vorgelegt von: Belinda Woppowa an der Hochschule der Medien Stuttgart am 06. Februar 2018, 2018. http://hdl.handle.net/10760/32596 (13.03.2019).

Wuthe, Cortina [2017]: Ergebnisse der Umfrage zum RDA-Katalogisierungslevel und weiterer Planungen. Stadtbibliothek Steglitz-Zehlendorf. Universitäts- und Landesbibliothek Münster. Münster, 08.09.2017.

http://www.aibm.info/wp-content/uploads/2017/09/2017-09-08_RDA_MusKat_VortragAIBM-2017.pdf (09.03.2019). 


\section{Anhang 1: Online-Fragebogen}

\section{Umfrage zur Katalogisierungssituation in den Öffentlichen Bibliotheken Sachsen-Anhalts}

Vielen Dank, dass Sie sich Zeit für den Fragebogen nehmen.

Im Rahmen meiner Masterarbeit im Studiengang Bibliotheksinformatik an der Technischen Hochschule Wildau führe ich eine Umfrage zur derzeitigen Katalogisierungssituation in den hauptamtlich geleiteten Öffentlichen Bibliotheken unseres Bundeslandes durch.

Hauptziel ist das Kenntlichmachen der Situation verbunden mit dem Versuch, Anleitungen und Wege zur Verbesserung zu geben. Im Mittelpunkt der Umfrage stehen die Nutzung von Fremddaten und die Möglichkeiten der einzelnen Bibliothek, diese einsetzen zu können.

Die Umfrage wird ca. 15 - 20 Minuten dauern. Mit den Pfeiltasten rechts unten können Sie zurückspringen.

Als Bibliotheksleitung werden Ihnen alle zutreffenden Fragen angezeigt. Bei Auswahl 'Mitarbeiter der Katalogisierung' beginnt die Umfrage ab Frage Nr. 19 und bezieht sich auf die konkrete Katalogisierungssituation der Kollegin/ des Kollegen.

Bei der Durchführung und Auswertung gelten die Bedingungen der Datenschutzverordnung (DSGVO).

Einschließlich der Frage Nr. 24 werden die Daten anonym erhoben und ausgewertet.

Die identifizierenden Angaben in Frage Nr. 25 und 26 (Name, E-Mail, Anschrift, Bibliotheksmanagementsystem) werden für eine mögliche Kontaktaufnahme im Anschluss an diese Arbeit, sowie bei der Teilnahme am Gewinnspiel erhoben. Diese Daten werden separat von den anderen Antworten gespeichert und können von mir nicht mit den vorherigen Fragen in Verbindung gesetzt werden.

Ich würde mich freuen, wenn Sie mich mit Ihren Antworten unterstützen könnten.

Andrea Kiefer TH Wildau

\section{Bereich: Grundsatzfragen}

1. Um die Fragen auf Ihre Funktion in der Bibliothek zuschneiden zu können, geben Sie bitte Ihren Aufgabenbereich in der Bibliothek an.

Hinweis: Sind Sie Bibliotheksleitung UND Katalogisierer - wählen Sie bitte 'Bibliotheksleitung'.

o Bibliotheksleitung o Mitarbeiter in der Katalogisierung

2. Bitte geben Sie die letzte Ihnen bekannte Zahl aus einer offiziellen Statistikquelle, wie dem Einwohnermeldeamt an.

o bis 10.000 Einwohner

o 20.001 - 30.000 Einwohner

0 10.001 - 20.000 Einwohner

o 40.001 - 50.000 Einwohner

o 30.001 - 40.000 Einwohner

Bemerkungen:

3. Wie viele Mitarbeiter/-innen sind in Ihrer Bibliothek beschäftigt (DBS-Werte)

Bitte geben Sie die Vollzeitäquivalente (VZÄ) Ihrer letzten DBS-Meldung an. Bitte füllen Sie alle Felder aus.

Fachbibliothekar/-innen $\mathrm{VZÄ}$

.....darunter in der Katalogisierung tätig:

VZÄ

Fachangestellte/ Bibliotheksassistent/-innen $V Z \ddot{A}$

.....darunter in der Katalogisierung tätig: ___ _ _ _ _ _ _ _

sonstiges Personal

.....darunter in der Katalogisierung tätig: _ _ _ _ _ _

Möchten Sie hierzu etwas ergänzen oder erläutern.:

4. Welches Bibliotheksmanagementsystem setzen Sie in Ihrer Bibliothek ein? Gemeint ist die Software Ihres Ausleih- und Katalogsystems.
o BIBLIOTHECAplus
o WinBIAP
o allegro-OEB
o LIBRARY for Windows

o Sonstiges:

Setzen Sie weitere Software im Bereich der Katalogisierung ein, dann listen Sie diese bitte hier auf - z.B. ein zusätzliches Katalogisierungstool o.ä.:

5. Setzen Sie für Ihre Bibliotheksnutzer einen Katalog/ OPAC bzw. ein Discovery-System ein?

o Ja o Nein

Bitte geben Sie die Bezeichnung ein (WWW-OPAC; OPEN; allegro-OPAC...) 
6. Nutzen Sie Katalogerweiterungen (Catalogue Enrichment) in Ihrem OPAC / Discovery System und / oder bieten Sie RSS-Feeds an? Gemeint sind z.B. Coverabbildungen, zusätzliche Links zu Wkipedia, Links zu Open Access Materialien, BibTip, Inhaltsverzeichnisse, RSS-Feed für Neuerwerbungen ...
o Ja o Nein
o wir bieten keinen OPAC an

Listen Sie die eingesetzten Anwendungen bitte auf:

7. Planen Sie als Bibliothek in nächster Zukunft ein cloudbasiertes Bibliotheksmanagementsystem einzusetzen? Bsp. LMSCloud

○ ja, in ca.___Jahren o nein, darüber denken wir noch nicht nach o dazu kann ich nichts sagen Aus welchen Gründen möchten Sie auf ein cloudbasiertes System wechseln?

\section{Bereich: IT/ Anbindung}

8. Katalogisierung - Schnittstellen, Normdateien Hierzu möchte ich Ihnen noch folgende Erklärungen geben:

GND- > in der Gemeinsamen Normdatei sind die Personennamendatei (PND), Gemeinsame Körperschaftsdatei (GKD), Schlagwortnormdatei (SWD) und die Einheitssachtitel-Datei des Deutschen Musikarchivs (DMA-EST-Datei) zusammengeführt

Schnittstellenformat /-protokoll -> Internetprotokoll über welches die Katalogdaten abgefragt werden können Datenformat -> maschinelles Austauschformat für Katalogdaten
Verwenden Sie die GND-Normdateien der DNB?
o ja
o nein o nicht bekannt

Welche Katalogisierungsregeln werden bei Ihnen angewendet?

$\square$ RAK-ÖB $\square$ RAK-WB $\square$ RAK-ÖB / RDA Mix

$\square$ RDA $\square$ nicht bekannt

Besitzt Ihre Bibliothek einen Z39.50 Client für Katalogimporte? o ja o nein o nicht bekannt

Welches Schnittstellenformat oder-Protokoll nutzen Sie für Katalogimporte? $\square$ Z39.50 $\square$ SRU $\square$ OAI

$\square$ andere $\square$ nicht bekannt

Welches Datenformat wird in Ihrem Bibliothekskatalog für Neuaufnahmen verwendet?

$\square$ MAB2 $\square$ MARC21 $\square$ BIBFRAME $\square$ andere

Bei Auswahl 'andere' geben Sie dieses Schnittstellen- oder Datenformat bitte hier an.

9. Wenn Sie MARC21 verwenden, wurden die alten Titelaufnahmen in MARC21 konvertiert?

o Ja o Nein o weiß ich nicht

Möchten Sie dazu etwas ergänzen?

10. Wer betreut in Ihrer Bibliothek die Hardware und die Server?

Wer ist zuständig für Wartung, Updates, Softwarevorhaltung der PC-Technik und des Datenservers Ihres Bibliotheksmanagementsystems? Mehrfachantwort möglich.

$\square$ die IT-Abteilung der Stadt- bzw. Gemeindeverwaltung - bzw. deren zuständige IT-Firma

$\square$ eine Verbundzentrale - welche:

$\square$ der Systemanbieter als Hosting $\square$ eine andere Bibliothek

$\square$ Ihre Bibliothek selbständig $\square$ das ist mir nicht bekannt $\square$ Sonstiges (bitte angeben)

Bei Mehrfachantworten erläutern Sie dies bitte kurz:

11. Betreiben Sie in Ihrer Bibliothek eine zweite Datenbank mit Katalogdaten?

Gemeint ist eine 2. Datenbank in der Sie regelmäßige Lieferungen eines oder mehrerer Anbieter für die Katalogisierung vorhalten, um sie in Ihre Echtdatenbank importieren zu können.

o Ja o Nein

Möchten Sie etwas ergänzen? $\square$ Dazu kann ich nichts sagen.

\section{Bereich: ÖVK}

12. Nimmt Ihre Bibliothek am ÖVK teil? ÖVK = Verbundkatalog der Öffentlichen Bibliotheken im GBV o Ja o Nein o ausgestiegen

Bitte teilen Sie uns Ihre Gründe für den Ausstieg und das Ausstiegsjahr mit:

$\square$ Keine Angabe

13. Warum nehmen Sie nicht am ÖVK teil? (Nur angezeigt, wenn Frage 12 mit ,nein' beantwortet worden ist) Mehrfachantwort möglich.

$\square$ kein Mehrwert $\square$ zu inaktuell

$\square$ fehlendes Know-how $\square$ damit haben wir uns noch nicht beschäftigt

$\square$ Dazu kann ich nichts sagen.

14. Wenn eine laufende - möglichst automatisierte - Aktualisierung der Katalogdaten dazu führen würde, dass Sie aktuelle Titeldaten direkt aus dem ÖVK importieren könnten, würde Ihre Bibliothek dann dem ÖVK beitreten? 
Bitte geben Sie hier Ihre persönliche Meinung wieder.

o Ja o Nein $\quad$ Keine Angabe

Können Sie diese Antwort begründen?

\section{Bereich: Fremddaten allgemein}

15. Welche Arten von Fremddaten bezieht Ihre Bibliothek?

Gemeint sind Daten, die Sie über Schnittstellen erhalten bzw. aus dem Internet abrufen. Mehrfachantwort möglich. $\square$ kostenpflichtige Fremddaten (incl.ekz-ID) $\quad$ kostenfreie Fremddaten $\square$ Wir beziehen keine Fremddaten

Möchten Sie etwas ergänzen?

$\square$ dazu kann ich nichts sagen

16. Welche Anbieter von Katalogdaten bzw. welche Werkzeuge nutzen Sie in Ihrer Bibliothek zur Katalogisierung per Fremddatenübernahme? Bitte nennen Sie hier die Anbieter, bei denen Sie über Schnittstellen die Daten erhalten bzw. aus dem Internet ziehen. Mehrfachantwort ist möglich.

$\square$ DNB $\square$ ekz $\square$ Hugendubel

$\square$ Bibliotheksverbünde $\square$ Zack-Gateway $\square$ DIVIBIB für Onleihe-Titel

$\square$ Sonstiges

Welche Bibliotheksverbünde nutzen Sie (BVB, GBV, hbz, HeBIS, KOBV, SWB, VÖBB) bzw. welche sonstigen Quellen nutzen Sie zur Titelaufnahme?

$\square$ Wir nutzen keine Fremddaten

17. Wie viele Exemplare haben Sie in Ihrer Bibliothek in den genannten Mediengruppen 2018 erworben? Bitte schätzen Sie die Zahlen, oder geben die DBS-Zahlen von 2017 an, wenn sich die Zahlen nicht erheblich unterscheiden.

Printmedien Belletristik __Exemplare

Printmedien Kinder/ Jugendliteratur___Exemplare

Printmedien Sachmedien Exemplare

Möchten Sie etwas ergänzen?

Non-Book-Medien Exemplare

18. Wenn Sie Ihren tatsächlichen Medienetat 2018 (Medien + Zeitschriften - ungeachtet der dahinterliegenden Kostenstelle) zusammenrechnen - wieviel Prozent davon geben Sie ungefähr für kostenpflichtige Fremddaten aus? $\square$ kostenpflichtige Fremddaten (inkl. ekz-ID)

$\square$ sonstige kostenpflichtige Fremddaten $\%$

$\square$ wir nutzen keine kostenpflichtigen Fremddaten $\quad \square$ dazu kann ich nichts sagen

\section{Bereich: Katalogisierung individuell}

19. Welche Anbieter von Katalogdaten bzw. welche Werkzeuge nutzen Sie PERSÖNLICH zur manuellen Katalogisierung, indem Sie Daten kopieren und in Ihren Katalog einfügen? (dies ist Frage 2 für die Katalogisierer)

$\square$ DNB $\quad$ ekz $\square$ Hugendubel

$\square$ Bibliotheksverbünde $\quad \square$ Zack-Gateway $\quad \square$ jpc

$\square$ ich nutze keine Fremddaten $\square$ Sonstiges

Welche Bibliotheksverbünde nutzen Sie (BVB, GBV, hbz, HeBIS, KOBV, SWB, VÖBB) bzw. welche sonstigen Quellen nutzen Sie zur Titelaufnahme? $\square$ Ich katalogisiere nicht

20. Welche Katalogisierungs-Kategorien sind Ihnen bei einer Fremddatenübernahme persönlich wichtig (neben den Standardfeldern)?

Mehrfachantwort möglich.

$\square$ Annotation $\square$ Klassifikation $\square$ Schlagwort $\square$ weiterführende Links
$\square$ Sonstiges
Möchten Sie dazu etwas ergänzen?

21. Warum nutzen Sie persönlich keine Fremddaten? Mehrfachantwort möglich

$\square$ ich habe es noch nicht versucht $\quad \square$ erscheint mir zeitaufwändiger als eigene Katalogisate

$\square$ fehlendes Know-how bei der technischen Einrichtung in der Bibliothek

$\square$ wir möchten unsere gewohnte Katalogisierung beibehalten - das sind unsere Leser gewöhnt

$\square$ Qualität der Daten überzeugt mich nicht

$\square$ zu teuer $\quad \square$ Sonstiges

22. Wenn Sie die Gesamtanzahl der neu katalogisierten Medien des Jahres 2018 in den folgenden Mediengruppen einschätzen - wieviel Prozent haben Sie persönlich REIN MANUELL katalogisiert.

Bitte beziehen Sie die bitte Titel ein, die Sie vollständig per Hand katalogisiert haben UND die Titel, die Sie per Kopieren und Einfügen aufgenommen haben.

Bitte beantworten Sie jede Zeile. 


\begin{tabular}{|l|l|l|l|l|l|}
\hline Medienart & $1-25 \%$ & $26-75 \%$ & $76-100 \%$ & $\begin{array}{l}\text { nicht 'meine' } \\
\text { Mediengruppe }\end{array}$ & Keine Angabe \\
\hline Printmedien Belletristik & & & & & \\
\hline Printmedien Sachbuch & & & & & \\
\hline Printmedien Kinder- u. Jugendbuch & & & & & \\
\hline Hörbuch-CD Erwachsene & & & & & \\
\hline Hörbuch-CD Kinder & & & & & \\
\hline Musik-CD Erwachsene & & & & & \\
\hline Musik-CD Kinder & & & & & \\
\hline DVD/ BluRay Erwachsene & & & & & \\
\hline DVD/ BluRay Kinder/ Jugendliche & & & & & \\
\hline
\end{tabular}

Möchten Sie hierzu etwas ergänzen?

$\square$ ich katalogisiere nicht

23. Für wie wichtig erachten Sie persönlich den Einsatz von Katalogerweiterungen (Catalog Enrichment) in Ihrem OPAC/ Discovery-System? Schieberegler 1 bis 10

Gemeint sind Coverabbildungen, BibTip, Links zu Wikipedia, Links zu open access Materialien oder Inhaltsverzeichnisse

$\begin{array}{llllllllll}1 & 2 & 3 & 4 & 5 & 6 & 7 & 8 & 9 & 10\end{array}$

ich sehe es für unsere Nutzer nicht als hilfreich an ich finde es sehr wichtig

Möchten Sie dies begründen?___ $\quad$ _ dazu kann ich nichts sagen

24. Wie empfinden Sie persönlich die derzeitige Katalogisierungssituation in Ihrer Bibliothek in Bezug: Bitte geben Sie hier Ihre rein persönliche Meinung wieder. Bitte beantworten Sie jede Zeile.

\begin{tabular}{|l|l|l|l|l|l|}
\hline & sehr schlecht & schlecht & Gut & Sehr gut & Keine Angabe \\
\hline auf Fremddatenübernahme? & & & & & \\
\hline $\begin{array}{l}\text { auf die Möglichkeit, digitale Medien aufnehmen } \\
\text { und somit anzeigen zu können? }\end{array}$ & & & & & \\
\hline $\begin{array}{l}\text { auf Schulung und Weiterbildung zum Thema } \\
\text { Katalogisierung? }\end{array}$ & & & & & \\
\hline $\begin{array}{l}\text { darauf, dass Bibliotheksnutzer Medien gut } \\
\text { auffinden können? }\end{array}$ & & & & & \\
\hline $\begin{array}{l}\text { auf Einbindung von Open Access Angeboten? } \\
\text { (z.B. einen Link auf den freien Text eines } \\
\text { klassischen Werkes) }\end{array}$ & & & & & \\
\hline
\end{tabular}

Möchten Sie hierzu noch etwas ergänzen?

\section{Bereich: Weiteres}

25. Um im Anschluss an die Masterarbeit mit Ihnen gemeinsam mit der Fachstelle oder / und dem ÖVK im Gespräch bleiben zu können, würde ich Sie bitten, mir folgende Informationen zu geben:

Diese Daten werden separat und somit anonym zur Umfrage erfasst. Eine Verbindung zu Ihren vorherigen Fragen ist daher nicht möglich.

Name der Bibliothek Kontakt E-Mail

Bibliotheksmanagementsystem (BIBLIOTHECAplus, allegro-OEB,...)

$\square$ Ich stimme zu, dass Frau Kiefer diese Daten für eine weitere Kontaktaufnahme verwenden darf.

$\square$ Ich möchte nicht über eventuelle Möglichkeiten oder Zusammenarbeit zum Thema Katalogisierung informiert werden.

26. Für Ihre vollständige Teilnahme bedanke ich mich mit der Teilnahme an einem kleinen Gewinnspiel.

Zu gewinnen sind 10 Pakete mit kleinen Helfern im Büroalltag.

Name der Bibliothek/ des Mitarbeiters _ Kontaktmailadresse

$\square$ Teilnahmebedingungen

PLZ Ort, Straße

$\square$ Datenschutz

Ich bedanke mich recht herzlich für die Beantwortung der Fragen und Ihre investierte Zeit! Gibt es noch Fragen zur Umfrage, oder möchten Sie mir noch etwas mitteilen, dann kontaktieren Sie mich sehr gern unter ...@....de.

Andrea Kiefer TH Wildau 


\section{Anhang 2: Fragen an Anbieter der Bibliotheksmanagementsysteme}

1. Welche Datenformate unterstützt Ihre Software für Import und Export von Katalogdaten (nur Mediendaten)?

$$
\begin{array}{ll}
\text { - } & \text { MAB2 } \\
\text { - } & \text { MARC21 } \\
\text { - } & \text { BIBFRAME } \\
\text { - } & \text { Sonstige: }
\end{array}
$$

2. Werden die Metadaten innerhalb der Software in ein internes Datenformat umgewandelt?

3. Bietet Ihre Bibliotheksmanagementsoftware ein Tool an, mit dem über die ISBN/ EAN eine direkte Datenübernahme mittels SRU und/oder Z39.50 Schnittstelle in MARC21 erfolgen kann? (Ziel ist die direkte Übernahme der Metadaten in das Katalogisierungstool bei Eingabe der ISBN/ EAN - ohne zwischengespeicherte Daten in einer zweiten Datenbank oder Datei)

4. Können mit diesem Tool die hier beschriebenen Services abgefragt werden?

https://verbundwiki.gbv.de/display/VZG/SRU

https://verbundwiki.gbv.de/display/VZG/Z39.50

https://services.dnb.de/sru/dnb?operation=explain\&version=1.1

5. Kann die Fremddatenübernahme von Katalogdaten in MARC21 und in MAB2 durchgeführt werden? Gibt es bei der Übernahme dieser beiden Formate Unterschiede (außer der Anzahl der Kategorien)?

- $\quad$ unterschiedliche Wege? (z.B. ist ein Zwischenspeichern notwendig?)

- $\quad$ können alle Kategorien übernommen werden, die in der Datenquelle für die zur Verfügung stehenden Kategorien vorhanden sind?

Kann ich als Anwender diese Daten filtern? (Kategorien ausblenden)

6. Können Titelaufnahmen mit Ihrem Bibliotheksmanagementsystem vollständig nach RDA (derzeitiger Stand) aufgenommen werden?

7. Gibt es Erfahrungen für die Konvertierung von Altdaten in MARC21? Sehen Sie einen Mehrwert darin, mit Beginn der Titelaufnahme in MARC21 auch die im eigenen Katalog der Bibliothek vorhandenen MAB2-Daten zu konvertieren? (bei einem System mit traditionellem OPAC)

Können Sie Unterschiede benennen, die für eine Konvertierung der Altdaten sprechen?

8. Stellen Sie für Ihr Importmodul konkrete Schritt-für-Schritt Anleitungen für die Fremddatenübernahme der verschiedenen Anbieter zur Verfügung? Können Sie die URL angeben oder mir Anleitungen zur Verfügung stellen und darf ich diese im Anhang der Arbeit veröffentlichen?

9. Ist eine konkrete Hilfestellung bei Problemen der Fremddatenübernahme Teil Ihres Serviceangebots, Teil der Wartungsverträge oder muss dies extra vergütet werden? Bieten Sie Schulungen / Webinare speziell zur Datenübernahme an?

10. Möchten Sie noch etwas zum Thema Fremddatenübernahme in Bezug auf Ihre Software mitteilen?

Andrea Kiefer, TH Wildau 
Anhang 3: Auswertungstabelle Expertenbefragung

\begin{tabular}{|c|c|c|c|c|}
\hline \multirow[t]{2}{*}{ Fragen } & \multicolumn{4}{|c|}{ Bibliotheksmanagementsoftware } \\
\hline & BIBLIOTHECAplus $^{189}$ & WinBIAP 190 & allegro-OEB ${ }^{191}$ & LIBRARY for Windows $^{192}$ \\
\hline $\begin{array}{l}\text { 1. Welche Datenformate unterstützt Ihre Software für } \\
\text { Import und Export von Katalogdaten (nur Mediendaten) } \\
\text { MAB2 } \\
\text { MARC21 } \\
\text { BIBFRAME } \\
\text { Sonstige }\end{array}$ & $\begin{array}{l}\text { ja } \\
\text { MARC21 } \\
\text { nein } \\
\text { UNIMARC, USMARC, CSV, SWU, } \\
\text { BIKAT }\end{array}$ & $\begin{array}{l}\text { ja } \\
\text { MARC21 - (Binär und XML) } \\
\text { nein } \\
\text { BIKAT, MAB1 }\end{array}$ & \multirow{2}{*}{$\begin{array}{l}\text { ja } \\
\text { MARC21 (Binär und XML) } \\
\text { nein } \\
\text { verschiedenste Anpassungen der } \\
\text { Formate, PICA3, BIKAT } \\
\text { ja - internes Format } \\
\text { an MAB2 angelehnt, aber eigenes } \\
\text { Format }\end{array}$} & \multirow[t]{2}{*}{$\begin{array}{l}\text { ja } \\
\text { MARC21 (Binär und XML) im } \\
\text { Import } \\
\text { nein } \\
\text { CSV, PDF, BIKAT, ASCII, DBASE } \\
\text { ja - internes Format }\end{array}$} \\
\hline $\begin{array}{l}\text { 2. Werden die Metadaten innerhalb der Software in ein } \\
\text { internes Datenformat umgewandelt? }\end{array}$ & ja - internes Format & $\begin{array}{l}\text { ja - internes Format } \\
\text { dieses erweitert z.B. für Klarschrift- } \\
\text { systematik }\end{array}$ & & \\
\hline $\begin{array}{l}\text { 3. Bietet Ihre Bibliotheksmanagement-software ein Tool } \\
\text { an, mit dem über die ISBN/ EAN eine direkte Datenüber- } \\
\text { nahme mittels SRU und/oder Z39.50 Schnittstelle in } \\
\text { MARC21 erfolgen kann? (Ziel ist die direkte Übernahme } \\
\text { der Metadaten in das Katalogisierungstool bei Eingabe } \\
\text { der ISBN/ EAN - ohne zwischengespeicherte Daten in } \\
\text { einer zweiten Datenbank oder Datei) }\end{array}$ & $\begin{array}{l}\text { ja - MAB2 in alte Erfassungsmaske } \\
\text { über Z39.50 direkt; } \\
\text { MARC21 - in neue Erfassungs- } \\
\text { maske - noch nicht dafür optimiert; } \\
\text { Z39.50 und SRU -für proprietäre } \\
\text { Schnittstelle der ekz; } \\
\text { neue RDA Maske soll erweitert } \\
\text { werden - dann Focus auf MARC21 }\end{array}$ & $\begin{array}{l}\text { ja - direkt in Medien-erfassung } \\
\text { ISBN ->über Z39.50 - dann Auswahl } \\
\text { im System und direkte Übernahme } \\
\text { (Z39.50 Client ist kostenlos } \\
\text { integriert) } \\
\text { MARC21 und SRU in neuer Version } \\
5.0\end{array}$ & $\begin{array}{l}\text { ja - } \\
\text { "allegro-OEB Fremddaten- } \\
\text { übernahme“ importiert MARC21- } \\
\text { Daten mittels SRU direkt in die } \\
\text { Katalog- oder Erwerbungs- } \\
\text { datenbank } \\
\text { Z39.50 wird nicht mehr ausgeliefert }\end{array}$ & $\begin{array}{l}\text { ja - direkt in Titelauf-nahme - } \\
\text { ISBN ->Daten importiert } \\
\text { mehrere Titel - Nutzung des } \\
\text { ZACK-Client in Datei }\end{array}$ \\
\hline Fragen & \multicolumn{4}{|c|}{ Bibliotheksmanagementsysteme } \\
\hline
\end{tabular}

189 Kiefer 01.02.2019b.

190 Kiefer 31.01.2019b.

${ }^{191}$ Kiefer 01.02.2019a.

192 Kiefer 31.01.2019a. 


\begin{tabular}{|c|c|c|c|c|}
\hline & BIBLIOTHECAplus & WinBIAP & allegro-OEB & LIBRARY for Windows \\
\hline $\begin{array}{l}\text { 4. Können mit diesem Tool die hier beschriebenen } \\
\text { Services abgefragt werden? } \\
\text { - https://verbundwiki.gbv.de/display/VZG/SRU } \\
\text { - https://verbundwiki.gbv.de/display/VZG/Z39.50 } \\
\text { - https://services.dnb.de/sru/dnb?operation=explain } \\
\text { \&version=1.1 }\end{array}$ & $\begin{array}{l}\text { SRU nicht möglich } \\
\text { aber Option Datenübernahme aus } \\
\text { Worldcat wird geprüft und } \\
\text { bevorzugt } \\
\text { nein } \\
\text { ja } \\
\text { nein }\end{array}$ & $\begin{array}{l}\text { SRU in Version } 5.0 \\
\text { ja - ab Version } 5.0 \text { abrufbar } \\
\text { ja - ab Version } 5.0 \text { abrufbar } \\
\text { ja - ab Version } 5.0 \text { abrufbar }\end{array}$ & $\begin{array}{l}\text { ja Fremddatenübernahme über SRU } \\
\text { für ÖVK, GVK, DNB } \\
\text { ja } \\
\text { nicht mehr } \\
\text { ja }\end{array}$ & $\begin{array}{l}\text { ja direkte Abfrage } \\
\text { dann als Datei importieren } \\
\text { ja } \\
\text { ja } \\
\text { ja }\end{array}$ \\
\hline $\begin{array}{l}\text { 5. Kann die Fremddatenübernahme von Katalogdaten in } \\
\text { MARC21 und in MAB2 durchgeführt werden? } \\
\text { Gibt es bei der Übernahme dieser beiden Formate } \\
\text { Unterschiede (außer der Anzahl der Kategorien)? } \\
\text { - unterschiedliche Wege? (z.B. ist ein } \\
\quad \text { Zwischenspeichern notwendig?) }\end{array}$ & $\begin{array}{l}\text { MAB2 - in alte Erfassungsmaske; } \\
\text { MARC21 - Nutzer wählt Erfassungs- } \\
\text { maske } \\
\text { MAB2 Import zusätzlich über } \\
\text { Zwischenablage möglich, } \\
\text { MARC21 über Import einer Datei } \\
\text { (über Schaltfläche) }\end{array}$ & $\begin{array}{l}\text { MAB } 2 \text { an ekz angelehnt; } \\
\text { MARC21 Anlehnung an DNB } \\
\text { keine unterschiedlichen Wege - } \\
\text { Datei wird angegeben - und Format } \\
\text { automatisch erkannt }\end{array}$ & $\begin{array}{l}2 \text { Wege möglich: } \\
\text { Fremddaten direkt über SRU } \\
\text { oder Import von Dateien }\end{array}$ & $\begin{array}{l}\text { Import über Datei - in beiden } \\
\text { Formaten möglich } \\
\text { nein }\end{array}$ \\
\hline $\begin{array}{l}\text { - können alle Kategorien übernommen werden, die } \\
\text { in der Datenquelle für die zur Verfügung stehenden } \\
\text { Kategorien vorhanden sind? } \\
\text { Kann ich als Anwender diese Daten filtern? (Kategorien } \\
\text { ausblenden) }\end{array}$ & $\begin{array}{l}\text { ja - } \\
\text { MAB2 - detailliert zu filtern } \\
\text { MARC21 - nur Klassifikation }\end{array}$ & $\begin{array}{l}\text { ja - Kategorien, die für öffentliche } \\
\text { Bibliotheken relevant sind } \\
\text { Kunde kann Kategorien filtern }\end{array}$ & $\begin{array}{l}\text { ja - alle benötigten Kategorien } \\
\text { Anwender kann filtern und } \\
\text { ergänzen, löschen und verändern }\end{array}$ & $\begin{array}{l}\text { ja - alle Felder können } \\
\text { dargestellt werden } \\
\text { Kategorien in Schulung } \\
\text { eingestellt - Kunde kann nicht } \\
\text { direkt filtern }\end{array}$ \\
\hline $\begin{array}{l}\text { 6. Können Titelaufnahmen mit Ihrem } \\
\text { Bibliotheksmanagementsystem vollständig nach RDA } \\
\text { (derzeitiger Stand) aufgenommen werden? }\end{array}$ & $\begin{array}{l}\text { die meisten Standard-elemente } \\
\text { sind erfassbar; Maske für ÖB } \\
\text { optimiert; Parallele Datensätze-> } \\
\text { noch nicht; } \\
\text { Werk- und Expressionsebene noch } \\
\text { nicht alle Standardelemente }\end{array}$ & alles integriert in Version 5.0 & $\begin{array}{l}\text { vollständig RDA - alle Pflicht- } \\
\text { kategorien sind abgebildet seit } \\
2015 \text {, optionale Kategorien - die, } \\
\text { die benötigt werden }\end{array}$ & $\begin{array}{l}\text { alle Felder können importiert } \\
\text { werden }\end{array}$ \\
\hline
\end{tabular}




\begin{tabular}{|c|c|c|c|c|}
\hline \multirow[t]{2}{*}{ Fragen } & \multicolumn{4}{|c|}{ Bibliotheksmanagementsysteme } \\
\hline & BIBLIOTHECAplus & WinBIAP & allegro-OEB & LIBRARY for Windows \\
\hline $\begin{array}{l}\text { 7. Gibt es Erfahrungen für die Konvertierung von } \\
\text { Altdaten in MARC21? Sehen Sie einen Mehrwert darin, } \\
\text { mit Beginn der Titelaufnahme in MARC21 auch die im } \\
\text { eigenen Katalog der Bibliothek vorhandenen MAB2- } \\
\text { Daten zu konvertieren? (bei einem System mit } \\
\text { traditionellem OPAC) } \\
\text { Können Sie Unterschiede benennen, die für eine } \\
\text { Konvertierung der Altdaten sprechen? }\end{array}$ & $\begin{array}{l}\text { keine Konvertierung } \\
\text { nur bei Migration } \\
\text { Anpassung ist in der } \\
\text { Katalogisierungsmaske intellektuell } \\
\text { möglich } \\
\text { nützliche Angaben für Leser (alle } \\
\text { Schauspieler auf einer DVD) - bsp. } \\
\text { für intellektuelle Anreicherung - für } \\
\text { Katalog -bleibt aber Einzelfall- } \\
\text { entscheidung }\end{array}$ & $\begin{array}{l}\text { Daten werden bei neuen Versionen } \\
\text { alle automatisch konvertiert; } \\
\text { bei Umstellung auf } 5.0 \text { - damit alles } \\
\text { auf RDA; } \\
\text { MAB2 -immer möglich - Daten } \\
\text { werden in MARC21 konvertiert }\end{array}$ & $\begin{array}{l}\text { keine Konvertierung Trennung } \\
\text { erfolgt durch Regelwerks-änderung }\end{array}$ & $\begin{array}{l}\text { keine Konvertie-rung durch } \\
\text { internes Format }\end{array}$ \\
\hline $\begin{array}{l}\text { 8. Stellen Sie für Ihr Importmodul konkrete Schritt-für- } \\
\text { Schritt Anleitungen für die Fremddatenübernahme der } \\
\text { verschiedenen Anbieter zur Verfügung? Können Sie die } \\
\text { URL angeben oder mir Anleitungen zur Verfügung } \\
\text { stellen und } \\
\text {...dürfen diese im Anhang der Arbeit veröffentlicht } \\
\text { werden? }\end{array}$ & $\begin{array}{l}\text { ja } \\
\text { Dokument Fremddatenübernahme } \\
\text { in alte Erfassungsmaske - ergänzt } \\
\text { Handbuch - FAQs über die } \\
\text { Webseite abrufbar (z.B. für DIVIBIB) } \\
\text { ja - im Anhang erlaubt }\end{array}$ & $\begin{array}{l}\text { ja } \\
\text { Handbuch } \\
\text { Handouts für Kunden - nicht im } \\
\text { Anhang } \\
\end{array}$ & $\begin{array}{l}\text { ja } \\
\text { Schritt-für-Schritt Anleitung als } \\
\text { Datei 'Fremddatenübernahme' } \\
\text { vorliegend, } \\
\text { Videoanleitung zusätzlich auf } \\
\text { Youtube } \\
\text { ja - im Anhang erlaubt }\end{array}$ & $\begin{array}{l}\text { Handouts für Kunden - nicht } \\
\text { im Anhang }\end{array}$ \\
\hline $\begin{array}{l}\text { 9. Ist eine konkrete Hilfestellung bei Problemen der } \\
\text { Fremddatenübernahme Teil Ihres Serviceangebots, Teil } \\
\text { der Wartungsverträge oder muss dies extra vergütet } \\
\text { werden? Bieten Sie Schulungen / Webinare speziell zur } \\
\text { Datenübernahme an? }\end{array}$ & $\begin{array}{l}\text { ja - Support unterstützt, } \\
\text { ist Teil des Supportvertrages - } \\
\text { Schulungen im Rahmen der } \\
\text { Katalogisierungs- oder Erwerbungs- } \\
\text { schulung }\end{array}$ & $\begin{array}{l}\text { ja - telefonischer Support und } \\
\text { Fernwartung } \\
\text { viele Kunden - in Cloud-wartung - } \\
\text { darin enthalten der Web-OPAC } \\
\text { (WinBIAPCloud) } \\
\end{array}$ & $\begin{array}{l}\text { ja - Support auf Anfrage, und } \\
\text { bei Wartungs-vertrag, Schulungen } \\
\text { vor Ort - Fremddaten-übernahme } \\
\text { wird geschult }\end{array}$ & $\begin{array}{l}\text { ja - Support/ Wartung bei } \\
\text { Einsatz des Importmoduls - } \\
\text { z.B. auch bei Änderungen }\end{array}$ \\
\hline $\begin{array}{l}\text { 10. Möchten Sie noch etwas zum Thema } \\
\text { Fremddatenübernahme in Bezug auf Ihre Software } \\
\text { mitteilen? }\end{array}$ & $\begin{array}{l}\text { Angebot wird ausgebaut, einfache } \\
\text { Übernahme im Vordergrund }\end{array}$ & $\begin{array}{l}\text { individuelles Fremddatenprofil je } \\
\text { Bibliothek, zusätzliche Inhalte wie } \\
\text { (Klarschrift, Mediengruppe) } \\
\text { kommen in nicht genutzte MAB- } \\
\text { Felder; } \\
\text { Buchhandel als Datenlieferant } \\
\text { Daten auch von kleinen } \\
\text { Bibliotheken online }\end{array}$ & $\begin{array}{l}\text { viele kleine Bibliotheken nutzen } \\
\text { DNB Daten, weil aktuell und sauber, } \\
\text { sowie nur } 1 \text { Treffer Integration der } \\
\text { Fremddatenübernahme direkt in } \\
\text { Katalogisie-rungsmodul ist } \\
\text { angedacht }\end{array}$ & $\begin{array}{l}\text { Einsatz der Konkordanz-liste } \\
\text { MAB2 auf MARC21 }\end{array}$ \\
\hline
\end{tabular}


11. Aus den Interviews hat sich die Frage nach der Möglichkeit Gespräche mit Hugendubel es dazu bei Ihnen Ansätze / Überlegungen?
Buchhändler werden immer mehr

zu Datenlieferan-ten (z.B.

Hugendubel, Pusted) kleinsten

Bibliotheken reicht VLB Sicht: Daten

direkt aus Buchhandels-

schnittstelle
VLB-Daten Übernahme noch nicht

Import-dateien im

Format überein-stimmen - ja 


\section{Anhang 4: Anleitung Datenübernahme aus dem Datenshop der DNB}

Für Datenübernahmen müssen Sie sich einmalig bei der DNB kostenfrei registrieren. Das können Sie hier tun. https://portal.dnb.de/myAccount/register.htm?method=showRegisterFormExtern\&view=redirect\%3A\%2FmyAccou nt\%2Fregister.htm\&dodServiceUrl=https\%3A\%2F\%2Fportal.dnb.de\%2Fdod

Mit dem erhaltenen Passwort loggt man sich dann hier ein:

https://services.dnb.de/dnb-cas/login?service=https\%3A\%2F\%2Fportal.dnb.de\%2Fj spring cas security check

Nach dem einloggen können Sie im Katalog Ihre Suchanfrage stellen.

https://portal.dnb.de/opac.htm?method=showSearchForm\#top

Ein Eintrag oder das Einlesen der ISBN gibt Ihnen einen konkreten Treffer. Liegt Ihnen die ISBN noch nicht vor, geben Sie wie im Beispiel Verfasser und Titel ein. Es ist eine automatische ,und' 'Verknüpfung hinterlegt. ${ }^{193}$

\section{KATALOG DER DEUTSCHEN NATIONALBIBLIOTHEK}

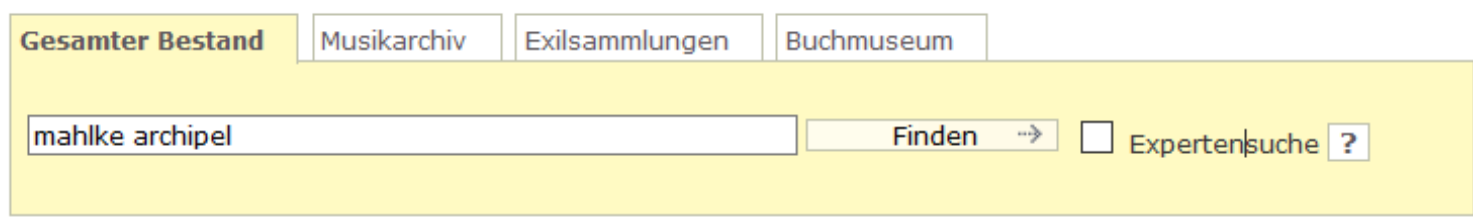

Nach Klick auf ,Finden' erhalten Sie Ihr Suchergebnis, so es im Bestand der DNB aufgenommen ist.

Ergebnis der Suche nach: "mahlke" and "archipel"

\& Zurück zur Trefferiste

tTreffer 3 von 4

\begin{tabular}{l}
\hline L \\
\hline Link zu diesem Datensatz \\
\hline Aftes Ihs
\end{tabular}

Art des Inhalts

Titel

Person(en)

Organisation(en)

Ausgabe

verlag

Zeitliche Einordnung

Umfang/Format

Andere Ausgabe(n)

ISBN/Einband/Preis

EAN

Sprache(n)

Sachgruppe(n)

Mit Klick auf ,In meine Auswahl übernehmen' wird dieser Vorgang durchgeführt. Das können Sie nun für alle Titel hintereinander erledigen, die sie aufnehmen wollen, oder die sie per Vorakzession für Ihre Fachreferat vorbereitet haben.

\begin{tabular}{|c|}
\hline http://d-nb.info/1156671760 \\
\hline Fiktionale Darstellung \\
\hline Archipel : Roman / Inger-Maria Mahlke \\
\hline Mahke, Inger-Maria (Verfasser) \\
\hline Rowohlt Verlag (Verlag) \\
\hline 1. Auflage \\
\hline Reinbek bei Hamburg : Rowohlt \\
\hline Erscheinungsdatum: September 2018 \\
\hline 429 Seiten ; $21 \mathrm{~cm}$ \\
\hline Erscheint auch als Online-Ausgabe: ISBN: 9783644001282 (2) \\
\hline $\begin{array}{l}\text { 978-3-498-04224-0 (9) Festeinband: EUR } 20.00 \text { (DE), EUR } 20.60 \text { (AT) } \\
3-498-04224-6 \text { (2) }\end{array}$ \\
\hline 9783498042240 (2) \\
\hline Deutsch (ger) \\
\hline
\end{tabular}

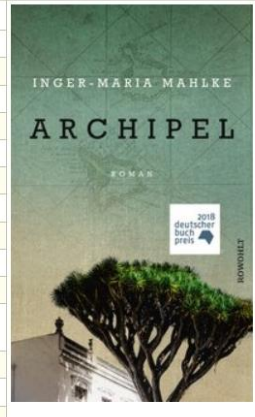

\section{尚 Aktionen}

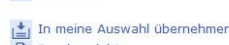
悬 Druckansicht

$\stackrel{\rightarrow=1}{\rightarrow}$ MARC21-XML-Repräsentation dieses Datensatzes 4 RDF (Turtle)-Reprasentation dieses Datensatzes $\rightarrow$ Dokumentation RDF (Linked Data Service) bf: BiBFRAME-Repräsentation dieses Datensatze $\rightarrow$ Projekt BIBFRAME
Korrekturanfrage

$\rightarrow$ buchhandel.de

193 Suchen Sie gezielter, bietet die DNB eine erweiterte Suche und eine Expertensuche an, die man mit dem Häkchen vor dem Feld aktiviert Danach erwartet das Eingabefeld die Kommandosprache CQL, die eine Suche differenziert ermöglicht. Hilfe: https://www.dnb.de/DE/Header/Hilfe/kataloghilfeExpertensuche.html 


$\begin{aligned} & \text { Katalog } \\ & \rightarrow \text { Einfache Suche } \\ & \rightarrow \text { Erweiterte Suche } \\ & \rightarrow \text { Browsen (DDC) } \\ & \rightarrow \text { Suchverlauf } \\ & \rightarrow \text { Meine Auswahl } \\ & \rightarrow \text { Hilfe } \\ & \rightarrow \text { Datenshop } \text { Mein Konto } \\ & \rightarrow \text { Mblieferung von } \\ & \text { Netzpublikationen } \\ & \rightarrow \text { Informationsvermittlung }\end{aligned}$

Mit Klick auf ,Datenshop' links im Menü gelangen sie dorthin. Als Datenformat sollten Sie hier MARC21 auswählen.

Der Datenshop zeigt Ihnen Ihre persönliche Auswahl an Titeln, die Sie nun mit dem Auswahlbutton ,Datensätze' bestätigen und mit Klick auf ,Auftrag starten' übernehmen.
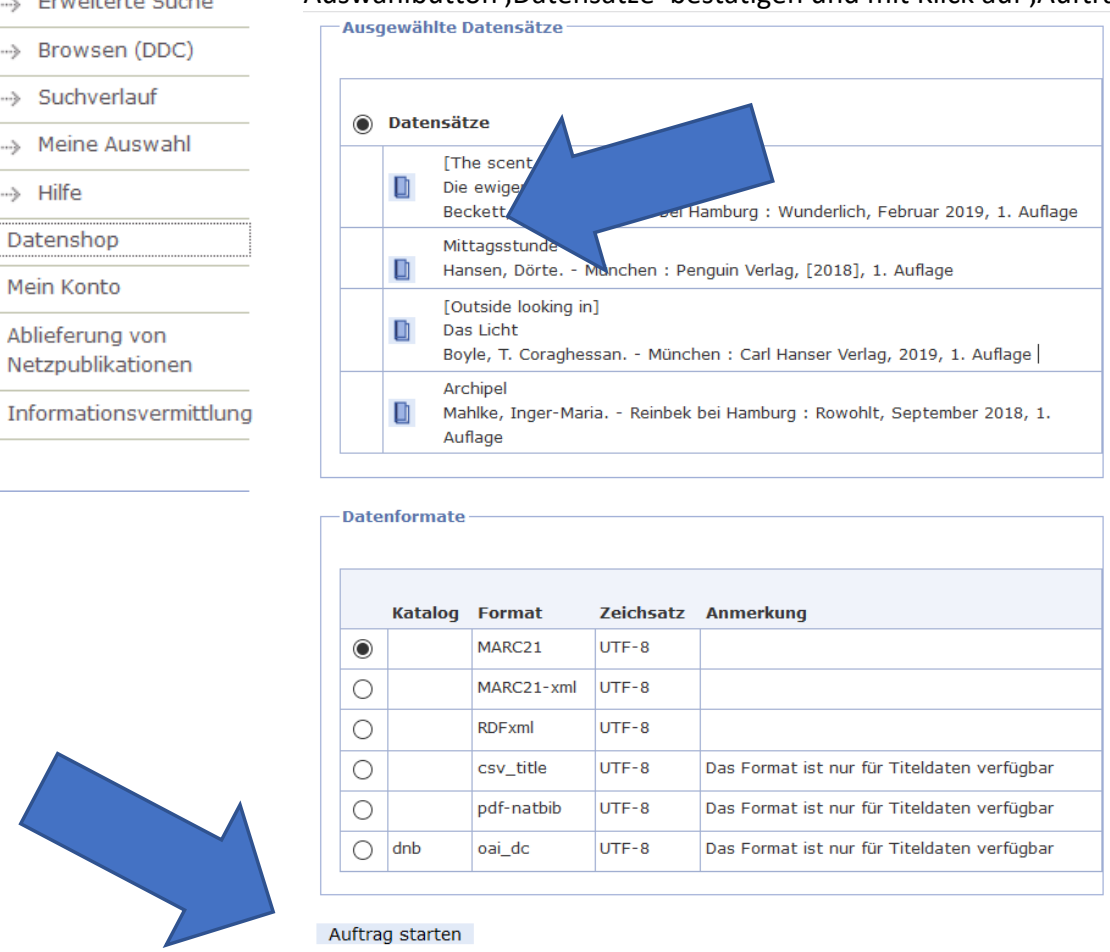

Auftrag starten

Sie gelangen danach in den Bereich: ,Liste der Aufträge'. Hier können Sie über den Link, download' die Daten direkt abspeichern und je nach Bibliotheksmanagementsystem weiterverarbeiten.

LISTE DER AUFTRÄGE

Bei einem Auftrag mit mehr als 800 Datensätzen kann die Bereitstellung mehrere Minuten dauern. Bitte aktualisieren Sie die Seite, falls Ihr Auftrag nicht angezeigt wird.

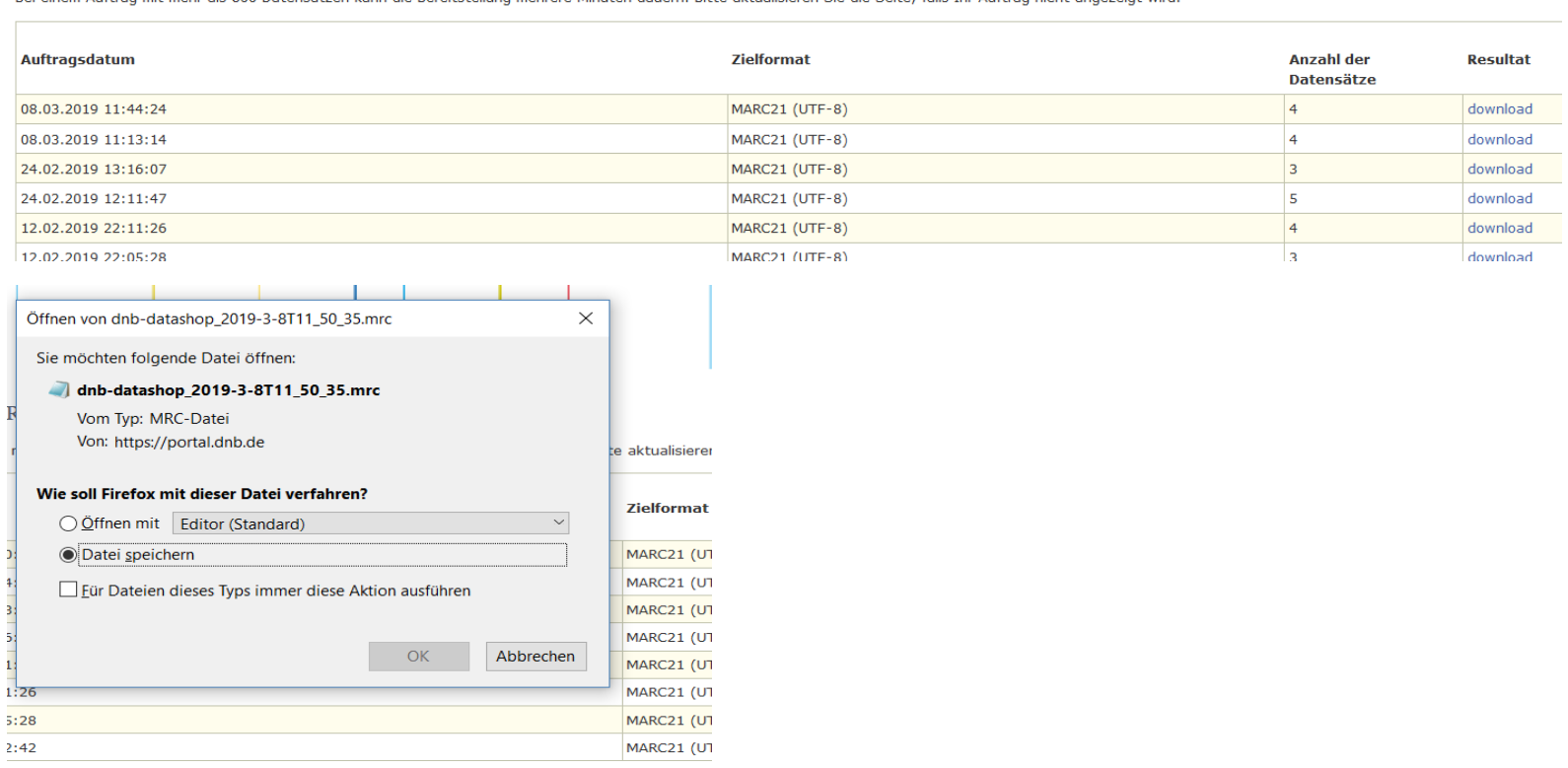

Sie sollten sich angewöhnen, nach dem Versenden der Daten die Titel in ,Meine Auswahl' zu entfernen. Sie müssen diesen Schritt sonst vor dem nächsten Export erledigen und verlieren bei vielen Titeln eventuell die Übersicht. Den Download aus der ,Liste der Aufträge' können Sie beliebig oft wiederholen. 


\section{Anhang 5: Anleitung Datenübernahme mit dem ZACK-Gateway}

Gesucht werden die Metadaten zum Titel Archipel von Mahlke. Vorausgewählt ist die Standardsuche mit Autor, Titel und ISBN. Alle Felder sind mit, und' verknüpft.

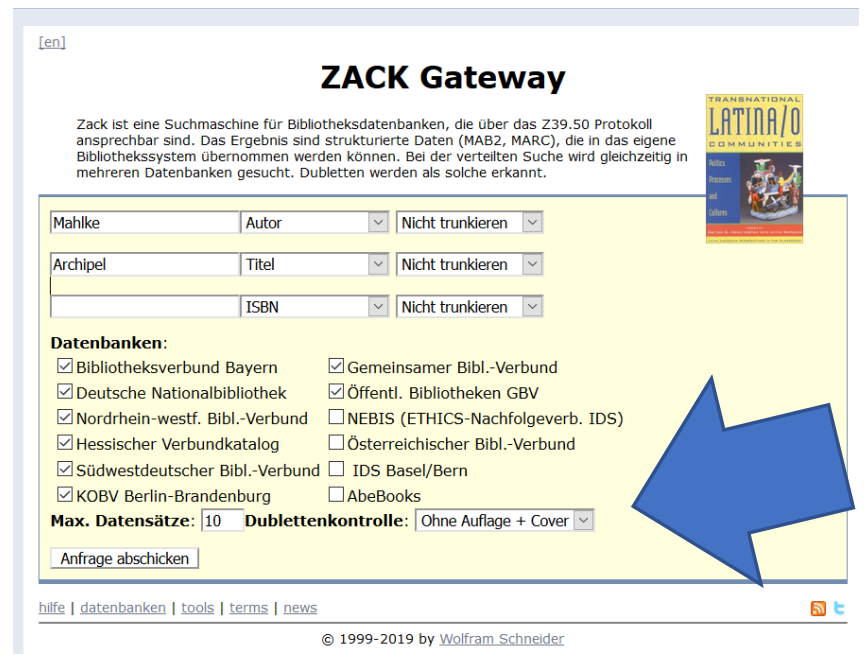

Ausgewählt sind die deutschen Kataloge, bei denen ein relevanter Treffer wahrscheinlich ist.

Als Zwischenergebnis erhalten wir eine Vorauswahl an Treffern.

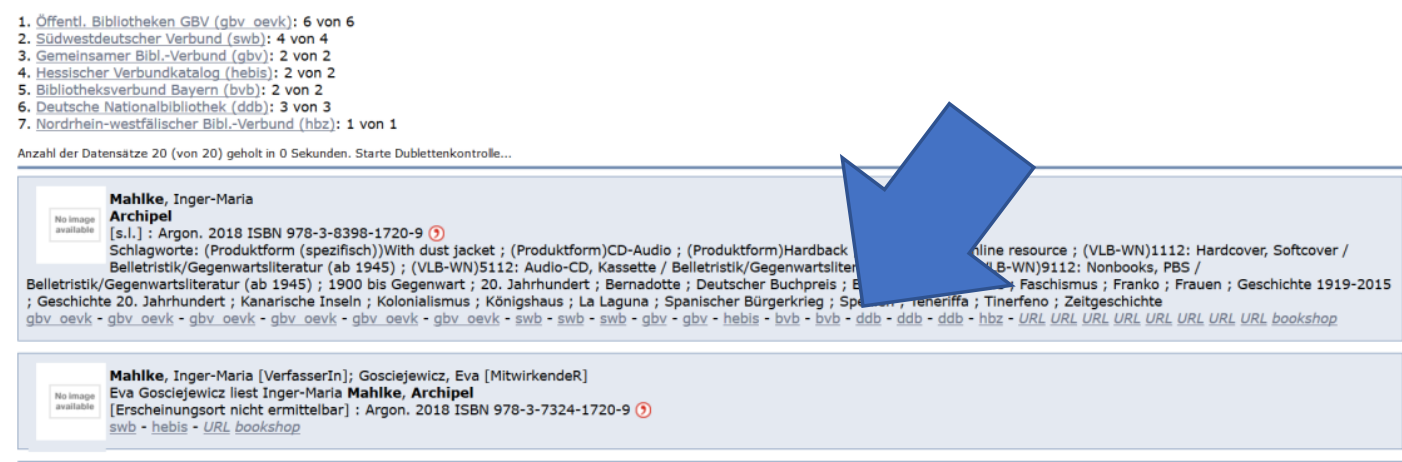

Die Treffer zeigen zu viele Portale an - hier sollten Sie bei Bedarf durch die Auswahl: Dublettenkontrolle z.B. noch auf die Auflage eingegrenzt werden.

\begin{tabular}{|c|c|}
\hline 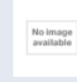 & $\begin{array}{l}\text { Mahlke, Inger-Maria } \\
\text { Archipel } \\
\text { [s.l.] : Argon. } 2018 \text { ISBN } 978-3-8398-1720-9 \text { (2) } \\
\text { gbv oevk - gbv - swb - swb - bookshop }\end{array}$ \\
\hline \multicolumn{2}{|c|}{$\begin{array}{l}\text { Mahlke, Inger-Maria } \\
\text { Archipel : Deutscher Buchpreis } 2018 \\
\text { Hamburg: Rowohtit Buchverlag. } 2018 \text { 5. Aufl. } \\
\text { gbv oevk bookshop }\end{array}$} \\
\hline 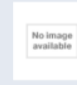 & $\begin{array}{l}\text { Mahlke, Inger-Maria } \\
\text { Archipel : Roman / Inger-Maria Mahlke } \\
\text { Reinbek bel Hamburg : Rowohit. 2018 3./4. Aufl ISBN 978-3-498-04224-0 (2) ISBN 3-498-04224-6 (2) } \\
\text { gbv oevk bookshop }\end{array}$ \\
\hline 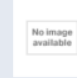 & $\begin{array}{l}\text { Mahlke, Inger-Maria } \\
\text { Archipel : Roman / Inger-Maria Mahlke } \\
\text { Reinbek beil Hamburg: Rowohlt. 2018 4. Auflage ISBN 978-3-498-04224-0 (2) } \\
\text { gbv oevk - bvb - bookshop }\end{array}$ \\
\hline 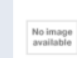 & $\begin{array}{l}\text { Mahlke, Inger-Maria } \text {-[Verfasser] } \\
\text { Archipel : Roman / Inger-Maria Mahlke } \\
\text { Reinbek bei Hamburg : Rowohlt. } 2018 \text { 1. Auflage ISBN 978-3-498-04224-0 (2) }\end{array}$ \\
\hline
\end{tabular}

Mit Klick auf das gewünschte Portal öffnet sich die Anzeige im vorausgewählten Datenformat. 
Anhang 5: Anleitung Datenübernahme mit dem ZACK-Gateway

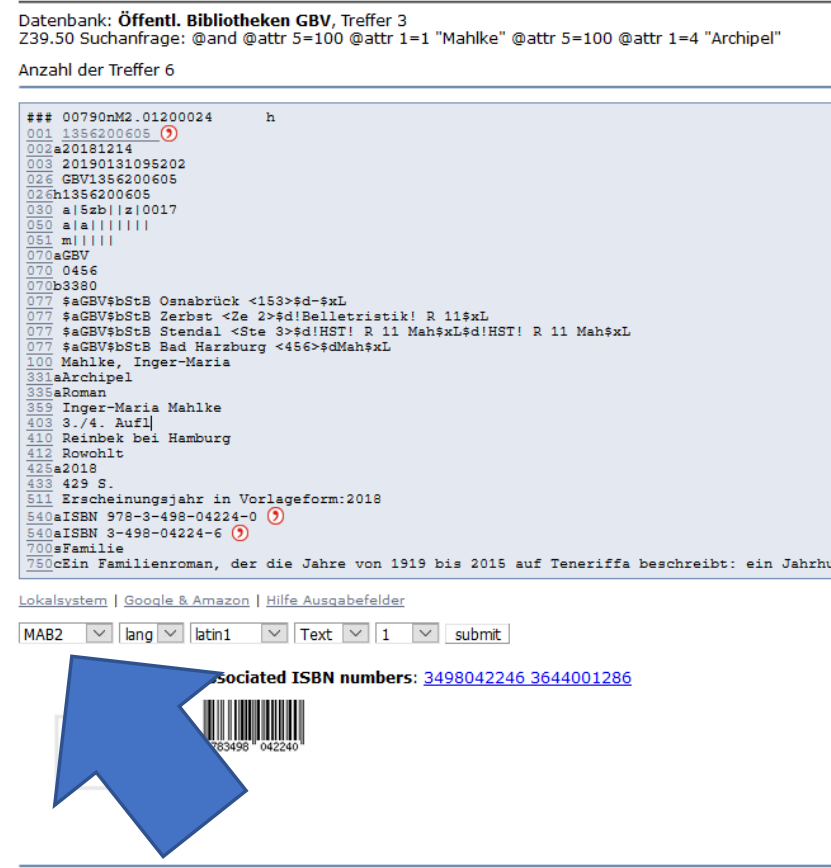

$\langle<1 \underline{1} \underline{2} 3 \underline{4} \underline{\underline{6}} \underline{[}[\gg>][S t e p$ ist 1$]$

Das Datenformat kann jetzt in den meisten Fällen noch gewechselt werden.

Gefällt Ihnen der Titel doch nicht, oder möchten Sie sehen, wie die anderen Aufnahmen aussehen, kann man unten die Treffer innerhalb des gewählten Portals wechseln.

Nun bleibt noch ein Klick auf den Datensatz, oder ein ,Markieren' und ,Kopieren' mit strg+C, um die Daten in die Zwischenablage zu bekommen (BIBLIOTHECAplus-MAB2), oder mit ,submit' abzuspeichern. 
Anhang 6: Konzeptvorschlag: Projekt Katalogisierung in Öffentlichen Bibliotheken Sachsen-

Anhalts

\section{Anhang 6: Konzeptvorschlag: Projekt Katalogisierung in Öffentlichen Bibliotheken Sachsen-Anhalts}

Im Fazit wurde ein Katalogisierungs-Projekt für die Öffentlichen Bibliotheken Sachsen-Anhalts vorgeschlagen. Die Kulturförderrichtlinie sieht eine Förderung für Projekte der Vernetzung zum Aufbau von effektiven Strukturen der bibliotheksmäßigen Versorgung vor.

Im Ergebnis dieser Arbeit werden dafür inhaltliche Schwerpunkte vorgeschlagen:

- Ansprechpartner für ÖVK, um Anbindungen aller eingesetzten Bibliotheksmanagementsysteme zu testen (Anleitungen für Datenimport und -export)

- gemeinsame Nutzungsmöglichkeiten von Kataloganreicherungen prüfen

- Überlegungen zur Einspielung oder Katalogisierung im Worldcat

- Klärung der Übernahmemöglichkeit der GND-Daten

○ gemeinsam zur Verfügung stehender Pool über den ÖVK

○ über die DNB oder

○ über das Linked-Open-Data-Angebot lobid ${ }^{194}$

- Prüfung der Möglichkeit, ob Daten des VLB oder Neuerscheinungsdienstes ${ }^{195}$ der DNB genutzt werden können

○ Gespräche/Zusammenarbeit mit DNB, um Übernahmeprofile für Öffentliche Bibliotheken zu erstellen - mit Neuerscheinungsdienst oder

- Gespräche mit dem GBV, ob Neuerscheinungsdienst der DNB bereitgestellt werden kann oder

○ Gespräche mit VLB, ob die REST-API für Käufe in der Bestellung genutzt werden kann

- Prüfung eines landesweiten Discovery-Systems

○ Zusammenarbeit mit dem GBV

○ oder/und Prüfung der bisher vorhandenen systemoffenen OPAC-Angebote

○ auch für kleine Bibliotheken, die bisher keinen OPAC vorhalten

\footnotetext{
194 Hochschulbibliothekszentrum des Landes Nordrhein-Westfalen o.J.https://lobid.org/gnd (13.03.2019).

195 siehe: Deutsche Nationalbibliothek 2019b.https://www.dnb.de/DE/Service/DigitaleDienste/ND/nd.html (11.03.2019).
} 
Anhang 6: Konzeptvorschlag: Projekt Katalogisierung in Öffentlichen Bibliotheken Sachsen-

Anhalts

- Regelmäßige Schulungsangebote zum Thema Katalogisierung, Datenformate, Schnittstellen, ÖVK/GBV, RDA für Öffentliche Bibliotheken

- Schulungsmaterial auch als Webinar oder Dokument, um alle Kolleginnen erreichen zu können (Reisekosten)

- Projektmitarbeiter für eine konkrete Betreuung der Bibliotheken, um Anbindung an Fremddatenangebote in der jeweiligen Bibliothek umsetzen zu können

○ und/oder Zusammenarbeit z.B. mit der HTWK - Studierende der Studienrichtung Bibliotheksinformatik in Praktika 


\section{Anhang 7: Optionen zur Fremddatenübernahme in BIBLIOTHECAplus ${ }^{196}$}

Inhalt
Übernahme von Daten für die (Bestell)Katalogisierung
Datenübernahme von der DNB
Datenübernahme aus einem Fremddatenpool
Import von Normdaten der DNB....
Datenübernahme aus dem Metadatenshop
Datenübübernahme vom BSZ (SWB)

\section{Vorbemerkung}

Da BIBLIOTHECAplus nicht Unicodefähig ist werden aktuell bei jeder Übernahme von Fremddaten im Zeichensatz UTF-8 (z.B. BSZ-Daten und DNB-Daten) einige Umlaute und Sonderzeichen in unpassende Sonderzeichen umgewandelt. Deshalb ist eine intellektuelle Kontrolle bei der Übernahme von Fremddaten angebracht.

\section{Übernahme von Daten für die (Bestell)Katalogisierung}

\section{Datenübernahme von der DNB}

\section{Datenübernahme aus einem Fremddatenpool}

BIBLIOTHECAplus erlaubt den Import von MARC21 Daten der DNB. OCLC schlägt vor die DNB Daten in einen Fremddatenpool (2. BIBLIOTHCA-Datenbank) zu importieren. So greift bei der Bestellkatalogisierung die Dublettenprüfung im Fremddatenpool und ermöglicht eine einfache Datenübernahme.

BIBLIOTHECAplus ermöglicht auch bei regelmäßigem Bezug von MARC21 Daten des Neuerscheinungsdienstes den regelmäßigen Import der Daten des Neuerscheinungsdienstes in den Fremddatenpool.

Die Lieferungen des Neuerscheinungsdienstes erscheinen in der Regel einmal wöchentlich und können beispielsweise auf einen FTP-Server erfolgen. Von dort wird die Datei einmal pro Woche manuell herunter geladen und manuell in den Fremddatenpool von BIBLIOTHECAplus importiert.

Weitere Informationen zum Bezug des Neuerscheinungdienstes der DNB finden Sie hier: http://www.dnb.de/DE/Service/DigitaleDienste/ND/nd.html

http://www.dnb.de/SharedDocs/Downloads/DE/DNB/service/bibliografischeDiensteFaltblattKost en. pdf? blob=publicationFile

http://www.dnb.de/datenshophilfe

Der Fremddatenpool kann zudem mit kostenfreien Fremddaten der DNB oder anderer Datenlieferanten befüllt werden um auch die Bestellkatalogisierung älterer Medien zu erleichtern. Zum Import einer MARC 21 Datei der DNB können Sie wie im Handbuch beschrieben vorgehen. Im Folgenden finden Sie den entsprechenden Auszug aus dem Handbuch von BIBLIOTHECAplus.

1. Starten Sie das Import-Modul und melden Sie sich an dem für den Import vorgesehenen (Fremd)DatenPool an.

2. Wählen Sie Assistent starten.

3. Wählen Sie auf der Registerkarte Format im Abschnitt Was für Daten möchten Sie einlesen? - MARCDaten als Datenformat MARC21 (DNB/ekz) aus.

\footnotetext{
196 Text und Grafiken original OCLC; - Größe angepasst
} 


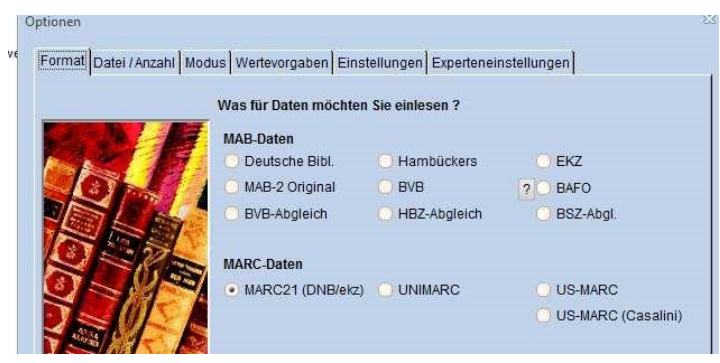

8. Kontrollieren Sie den angezeigten Dateinamen.

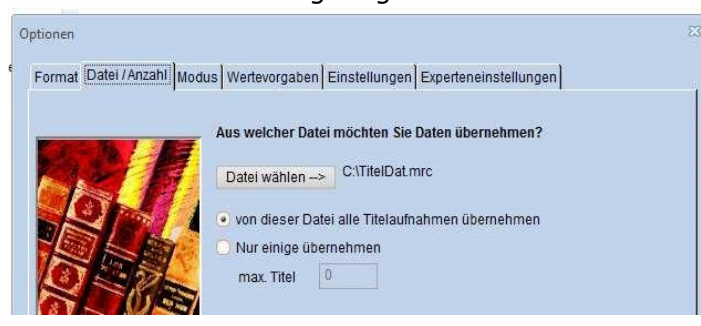

9. Wählen Sie für einen kompletten Import aller Datensätze der Datei die Option von dieser Datei alle Titelaufnahmen übernehmen aus. Wählen Sie für einen Teilimport die Option Nur einige übernehmen aus und spezifizieren Sie in dem Datenfeld max. Titel die Anzahl zu importierender Datensätze aus 10. Klicken Sie auf Weiter

11. Wählen Sie auf der Registerkarte Modus die von Ihnen gewünschten Import-Optionen bezüglich der Anlage von Medien aus.

12. Klicken Sie auf Weiter

13. Auf den Registerkarten Wertevorgaben und Einstellungen behalten Sie i. d. R. die Standardeinstellungen bei und klicken auf Weiter

14. Auf der Registerkarte Experteneinstellungen behalten Sie ebenfalls i. d. R. die Standardeinstellungen bei. 15. Klicken Sie auf OK

16. Wählen Sie Import starten in der rechten Menüleiste. Der Import wird gestartet 17.Nach dem erfolgreichen Import bestätigen Sie die Meldung über die Anzahl der importierten Medien mit OK

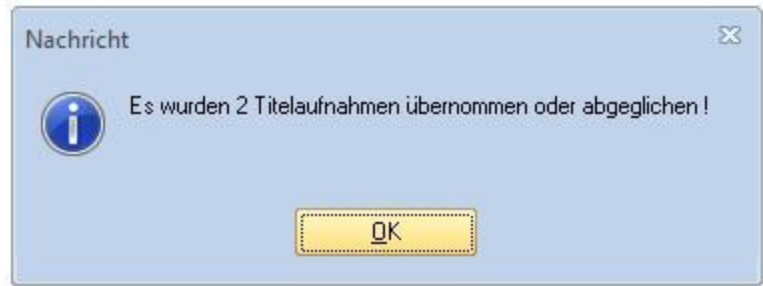

Zudem muss der Fremddatenpool als solcher in BIBLIOTHECAplus konfiguriert werden. Gehen Sie dazu wie folgt vor:

1. Anmeldung am Einstellungsmodul Ihrer Produktivdatenbank, wie gewohnt

2. Tragen Sie nun den Namen der Fremddatenbank im Parameter POOLS21 unter Konfiguration(AP) / Direkteinstellungen im Abschnitt Katalog ein und speichern Sie die Eingabe

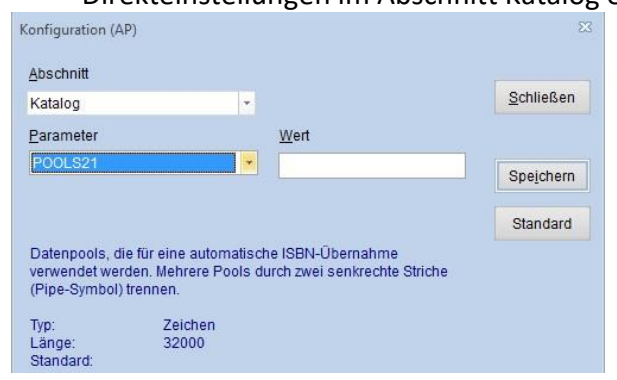

3. Um eine (Dienst)Recherche im Fremddatenpool zu ermöglichen, tragen Sie den Namen der Fremddatenbank im Parameter SEARCHPOOLS unter Konfiguration(AP) / Direkteinstellungen im Abschnitt All ein und speichern Sie die Eingabe 
Anhang 7: Optionen zur Fremddatenübernahme in BIBLIOTHECAplus

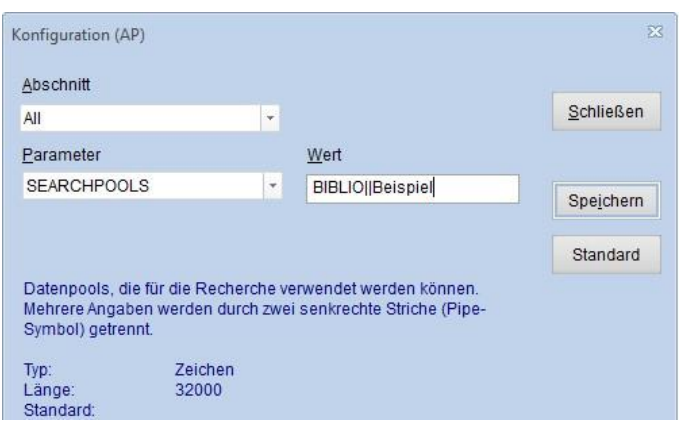

4. Starten Sie nun BIBLIOTHECAplus neu

Die eigentliche Datenübernahme erfolgt nun aus der Erfassungsmaske der Katalogisierung oder Erwerbung heraus. Sie funktioniert wie folgt:

1. Übernahme durch Dublettenmeldung

a. Aufruf einer Erfassungsmaske in der Katalogisierung oder Erwerbung

b. Eingabe einer ISBN oder eines Titels und ggf. eines Verfassers

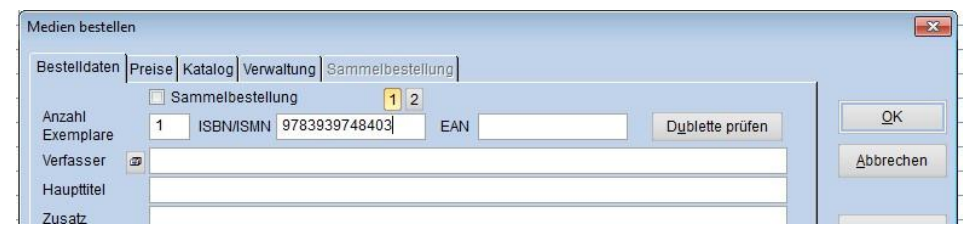

c. Verlassen des ISBN Feldes mit TAB oder Klick auf die Schaltfläche Dublette (ist bei Eingaben ohne ISBN unerlässlich)

d. eine Dublettenmeldung erscheint

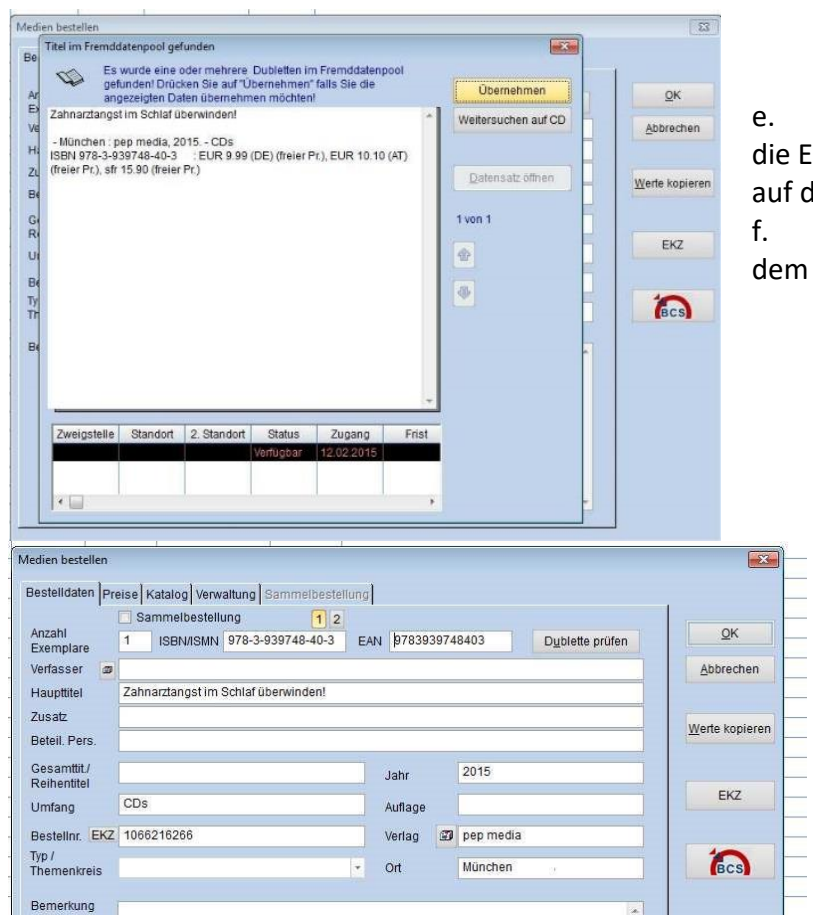

2. Übernahme durch Suche im Fremddatenpool

(Dazu muss der Fremddatenpool im Parameter SEARCHPOOLS hinterlegt sein)

a. Aufruf einer Erfassungsmaske in der Katalogisierung oder Erwerbung

b. Klick auf Werte Kopieren 
c. Klick auf Suche im Pool

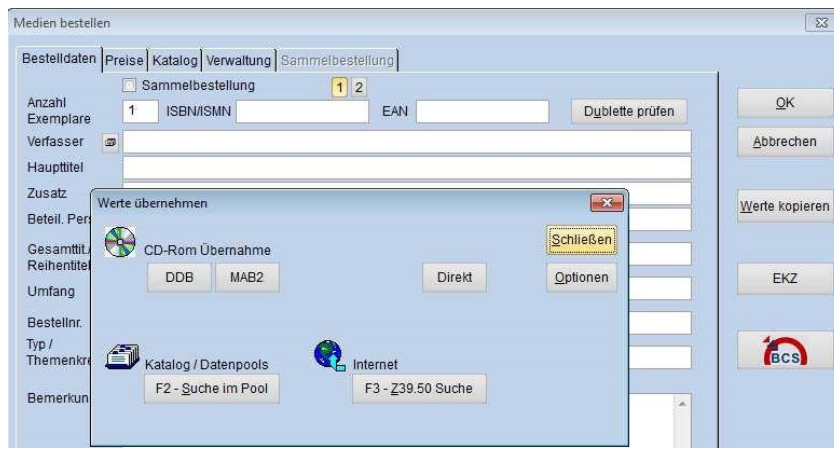

d. Auswahl des Fremddatenpools in der rechten unteren Ecke der Recherchemaske

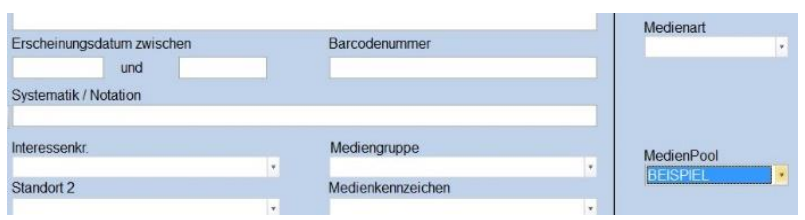

e. Durchführung der Recherche wie gewohnt

f. Auswahl des Datensatzes aus der Trefferliste

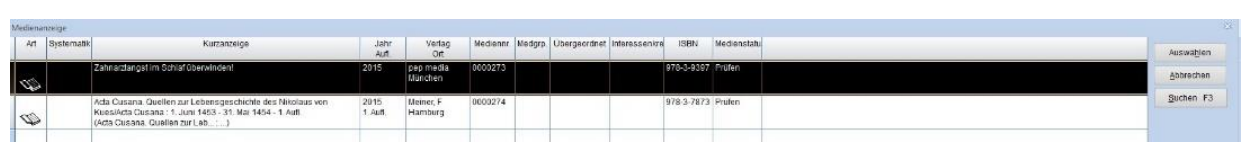

g. Übernahme der Daten in die Erfassungsmaske mit Doppelklick oder Klick auf die Schaltfläche Auswählen

h. Nun kann wie gewohnt mit dem Datensatz gearbeitet werden

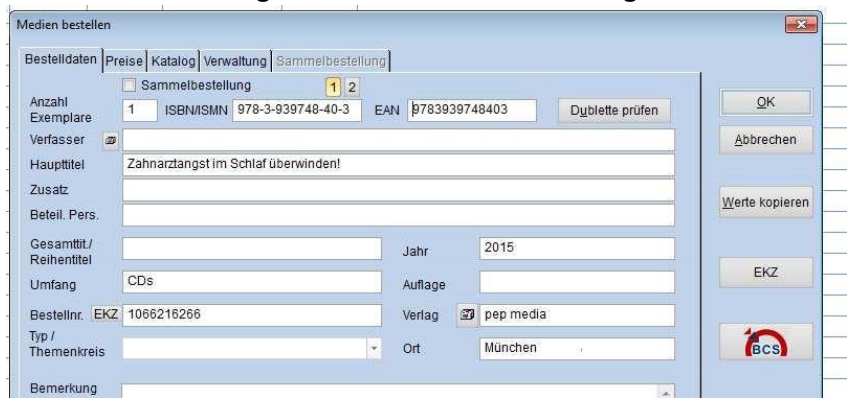

Datenübernahme aus dem Metadatenshop

Werden gezielt Daten bei der Bestellkatalogisierung aus dem Datenshop der DNB erworben empfehlen wir die Datenübernahme direkt aus der MARC21 Datei des Datenshops der DNB über die Schaltfläche DDB in der Erfassungsmaske unter Werte kopieren (Voraussetzung hierfür ist BIBLIOTHECAplus in der Version 5.0).

Das Vorgehen ist dazu wie folgt:

1. Anmeldung am DNB Metadatenshop.

2. Recherche des betreffenden Titels (Beispiel: http://d-nb.info/1066216266)

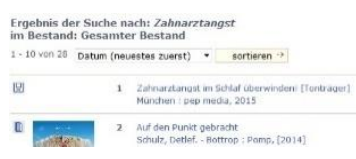

3. Übernahme des passenden Treffers in "Meine Auswahl" in der Detailansicht 


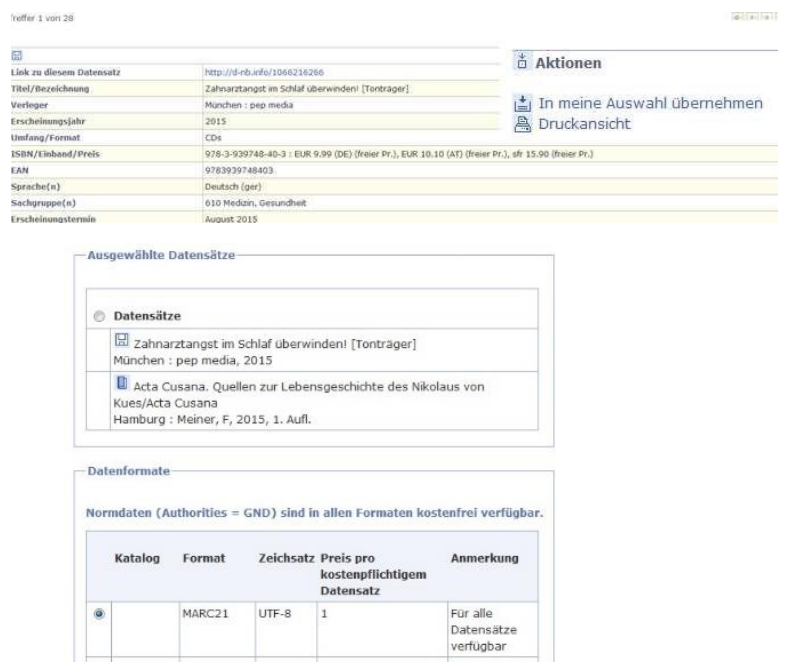

4. Schritte 2 und 3 können wiederholt werden (z.B. für http://d-nb.info/1061149609 )

5. Export der Auswahl in eine MARC21 Datei

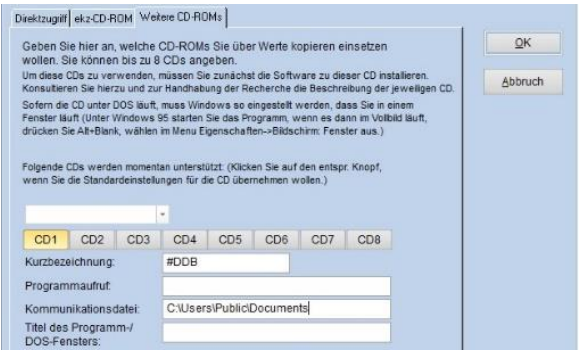

6. Ablage der Datei in dem unter Einstellungen / Konfiguration(AP) / CD-ROM für die weitere CD-ROM „DDB“ angegebenen Verzeichnis

7. Öffnen einer Erfassungsmaske in Katalogisierung oder Erwerbung

8. Klick auf Werte Kopieren

9. Klick auf die Schaltfläche DDB

10. Auswahl des gewünschten Titels und Klick auf die Schaltfläche Importieren
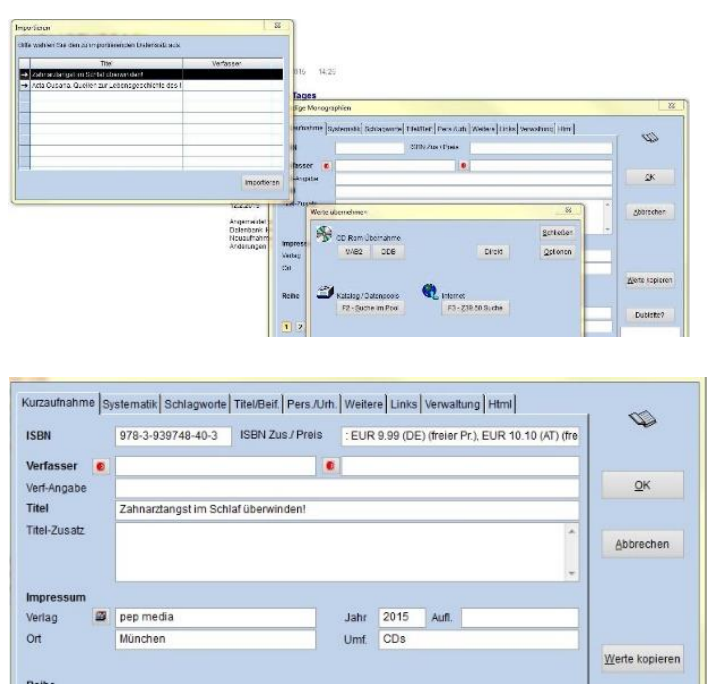

11. Dann kann der Datensatz wie gewohnt weiterbearbeitet werden

Datenübernahme vom BSZ (SWB)

Das BSZ ermöglicht die Übernahme der Daten des Datendienstes der DNB via Z39.50. Mittels der Z39.50 Schnittstelle können bei der Bestellkatalogisierung in der Erfassungsmaske unter Werte kopieren / Datensuche im Internet die jeweiligen Titeldaten vom Z39.50 Server des BSZ übernommen werden.

Das BSZ bietet über seinen Z39.50 Server auch Zugriff auf den Neuerscheinungsdienst der DNB.

Weitere Informationen finden Sie hier: 
https://wiki.bsz-bw.de/doku.php?id=v-team:daten:emailabo https://wiki.bsz-bw.de/doku.php?id=v-team:daten:z39.50

Zur Fremddatenübernahme über Z39.50 muss die Verbindung zum Z 39.50 Server des BSZ wie im Handbuch beschrieben konfiguriert werden. Der Z39.50 Client ist für diese Art der Datenübernahme unerlässlich. Er wurde von den Kornhausbibliotheken bereits erworben.

Die Datenübernahme gestaltet sich dann wie folgt:

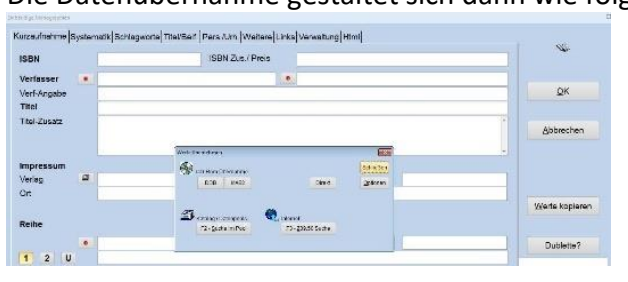

1. Aufruf einer Katalogisierungsmaske

2. Klick auf Werte Kopieren

3. Klick auf „F3 - Z 39.50 Suche“

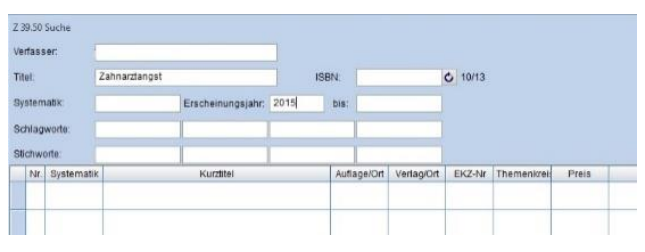

4. Nun erscheint eine Suchmaske

5. Hier kann nun der Z 39.50 Server des

BSZ zur Datenübernahme abgefragt werden

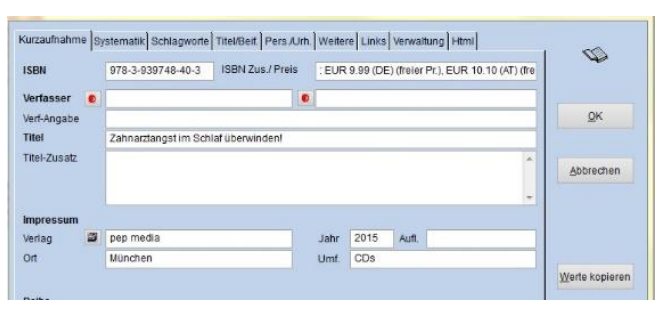

6. Mit Doppelklick oder Klick auf OK wird ein Datensatz in die Erfassungsmaske übertragen

7. Der Titel kann nun wie gewohnt weiter bearbeitet werden

\section{Datenübernahme von der ekz}

Die EKZ ermöglicht die Übernahme von Fremddaten auf mehreren Wegen. Diese sollen im Folgenden kurz angerissen werden.

1. Werden Medien im Medienshop der ekz bestellt ermöglicht die kostenfreie Schnittstelle zu den ekz Medienservices die automatische Übernahme der Bestelldaten mit grundständigem Katalogisat und wenn erworben, auch den umfangreichen Katalogdaten in BIBLIOTHECAplus.

2. Ab Version 5.1 von BIBLIOTHECAplus wird als Ersatz für den abgeschalteten Z39.50 Server der ekz eine sogenannte Rechercheschnittstelle in BIBLIOTHECAplus zur Verfügung stehen. Diese wird in der Erfassungsmaske unter Werte kopieren hinterlegt sein und sich ähnlich wie die Datenübernahme via Z39.50 verhalten.

3. Die ekz versendet zudem CD-ROMs mit Katalogdaten. Diese können in BIBLIOTHECAplus eingebunden werden. Sie verhalten sich ähnlich wie ein Fremddatenpool und ermöglichen die Datenübernahme mittels einer Dublettenmeldung.

\section{Datenübernahme vom BCS}

Für die Katalogisierung bereits erschienener Titel kann es lohnend sein die kostenfreie Datenübernahme aus dem BCS zu nutzen.

Gibt man eine ISBN in die Erfassungsmaske der Erwerbung oder Katalogisierung ein, ist es einfach möglich zu prüfen ob im BCS (BIBLIOTHECA Community System) die entsprechenden Katalogdaten vorhanden sind. Dazu wird dann einfach auf die Schaltfläche BCS geklickt. Alle BIBLIOTHECABibliotheken können an dieser Katalogisatetauschplattform partizipieren. Durch die Teilnahme von Bibliotheken jeder Größe schwankt allerdings die Qualität der Katalogisate deutlich. 
Der Versand von Fremddaten an das BCS wird automatisch von BIBLIOTHECAplus verhindert.

\section{Datenübernahme vom Zack Gateway (analog auch von Rezensionen.at)}

Aktuell bietet BIBLIOTHECAplus im Zuge der Datenübernahme aus der Zwischenablage die Option der kostenfreien MAB2 Datenübernahme vom Zack Gateway. Hierzu wird das entsprechende Medium im Zack Gateway recherchiert. Dann werden seine MAB-Daten aufgerufen und kopiert (mit Ctrl $+\mathrm{C}$ ). In BIBLIOTHECAplus werden diese in der Erfassungsmaske unter Werte kopieren mit der Schaltfläche MAB2 übernommen.

\section{Datenübernahme von der VLB CD}

BIBLIOTHECAplus bietet die Option Daten von der VLB-CD zu übernehmen. Ab Version 5.2 wird auch das neue Format der VLB-CD unterstützt. Sie können die VLB-Daten sowohl über eine Recherche auf der CD unter "Werte kopieren“ in die Erfassungsmaske übernehmen, als auch direkt, durch die Eingabe einer ISBN in der Erfassungsmaske.

Hinweis: Nahezu alle der oben beschriebenen Optionen können bei der Bestellkatalogisierung und auch bei der Einarbeitung eines Mediums zur Ergänzung des Bestellkatalogisats verwendet werden.

\section{Import von Normdaten der DNB}

Die Deutsche Nationalbibliothek stellt aktuell zweimal jährlich den aktuellen Stand der GND zur Verfügung. Auch dieser kann in einen Fremddatenpool importiert und als Normdatenbank in BIBLIOTHECAplus hinterlegt werden.

Wenn die GND regelmäßig komplett neu importiert wird empfehlen wir Ihnen hierzu stets eine neue Datenbank zu nutzen. Soll die GND einmalig als ergänzende Hilfestellung zur Katalogisierung genutzt werden kann sie auch einmalig in den Fremddatenpool für Titeldaten eingelesen werden.

Zum Import der GND kann die swdimp.exe genutzt werden. Dieses Werkzeug teilt die GND-Daten auf die Register von BIBLIOTHECAplus auf. Die swdimp.exe befindet sich standardmäßig im Verzeichnis ...BIBLIO_CLIENT\BIN.

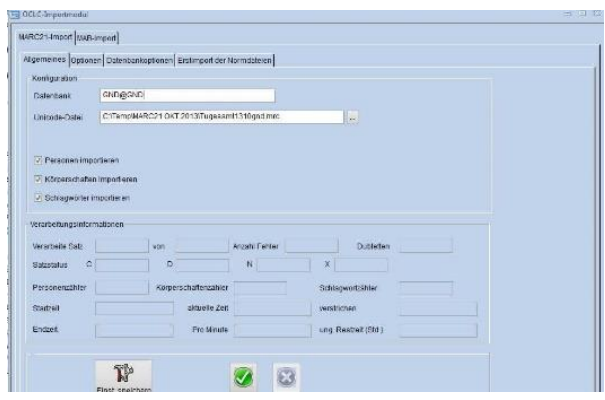

Nach erfolgtem Import kann die entsprechende Fremddatenbank in der Produktivdatenbank von BIBLIOTHECAplus als Normdatenbank konfiguriert werden. Dazu muss lediglich unter Einstellungen / Konfiguration(Sys) / Direkteinstellungen im Abschnitt Katalog in den Parametern RegDBPers, RegDBSchlag und RegDBUrh der Name der Normdatenbank hinterlegt werden.

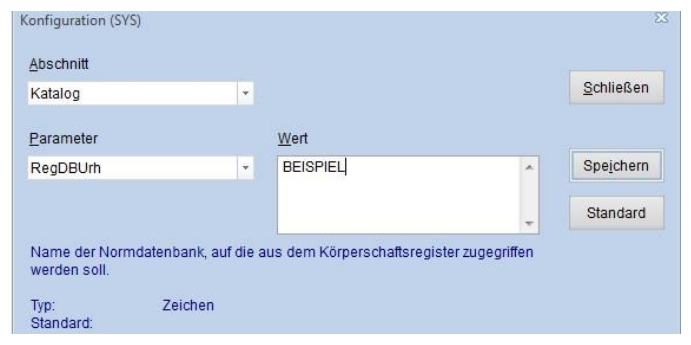

Nun kann, wenn in den eigenen Registern kein passender Eintrag gefunden wird, auf die importierten GND-Daten zugegriffen werden. 
Anhang 7: Optionen zur Fremddatenübernahme in BIBLIOTHECAplus

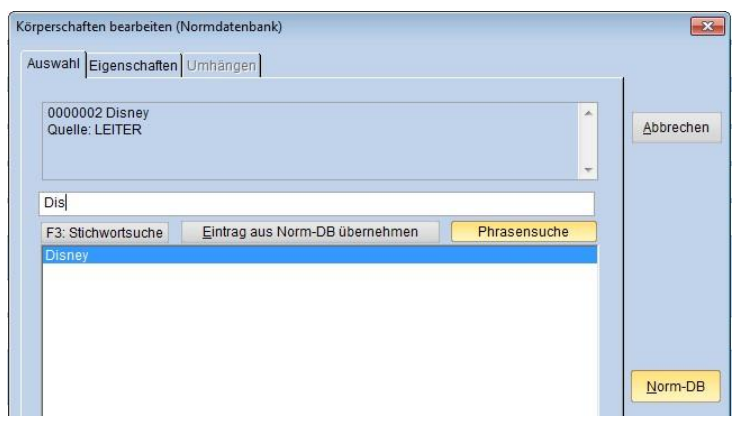

Der gewählte Eintrag wird in das Register der Produktivdatenbank übertragen und kann wie gewohnt verwendet werden. 


\title{
allegro-OEB Fremddatenübernahme
}

\section{Dokumentation}

\section{Büchereizentrale}

Niedersachsen

\author{
Büchereizentrale Niedersachsen \\ Lüner Weg 20 \\ 21337 Lüneburg \\ Telefon: 04131/9501-0 \\ Telefax: 04131/9501-24 \\ E-Mail: info@bz-niedersachsen.de
}

Stand: 29. November 2018

\section{Inhaltsverzeichnis}

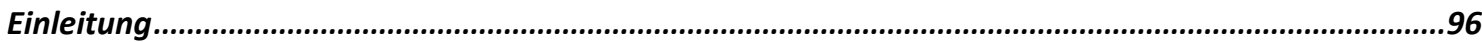

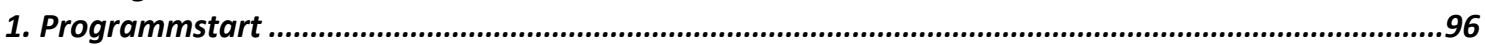

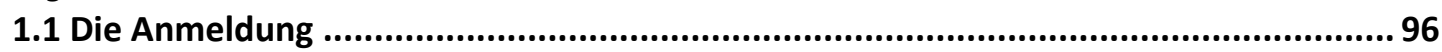

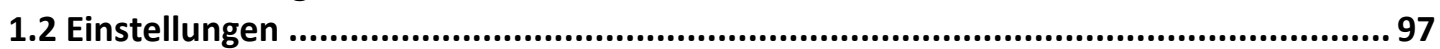

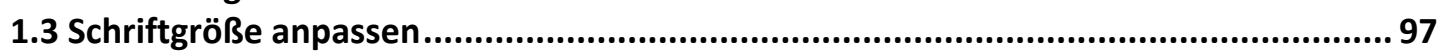

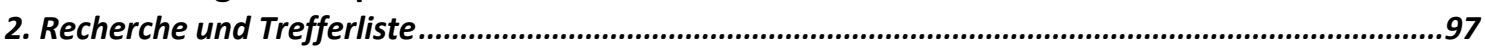

2.1 Treffermenge reduzieren ............................................................................. 98

2.2 Suchfilter einstellen ................................................................................. 98

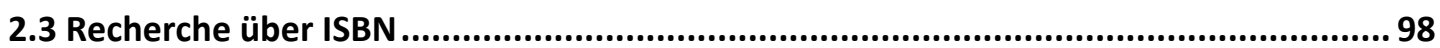

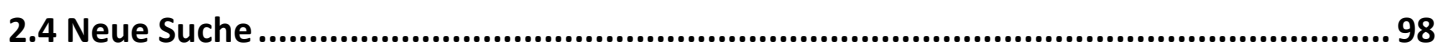

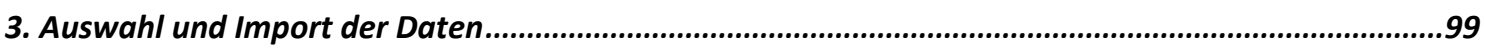

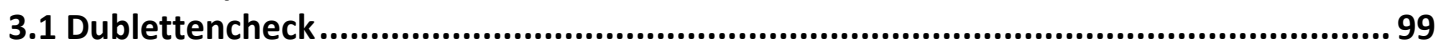

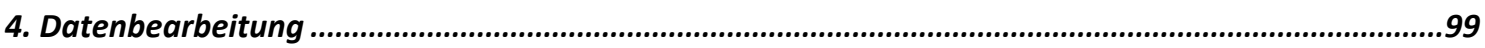

4.1 Bearbeitung im Programm „Fremddatenübernahme“ ............................................99 99

4.2 Bearbeitung im Programm ,Katalogisierung“ .................................................... 100

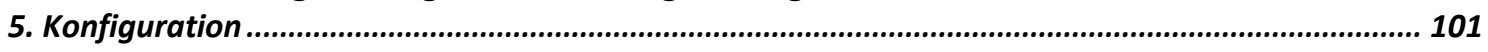

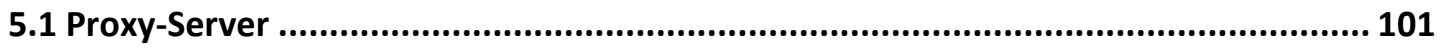

197 Text und Grafiken original Büchereizentrale Niedersachsen - Größe angepasst 


\section{Einleitung}

Diese Dokumentation beschreibt das Programm allegro-OEB Fremddatenübernahme. Es handelt sich hierbei um eine reine Anwenderdokumentation, die Ihnen die Einarbeitung in das Programm erleichtert.

Das Programm bietet Ihnen die Möglichkeit, in Bibliotheksdatenbanken im Internet nach Titeln zu recherchieren und diese in die eigenen allegro-OEB-Datenbanken („Katalog“ oder „Erwerbung“) zu übernehmen. Hierfür stehen Ihnen folgende Datenbanken als Datenquelle zur Verfügung:

- Katalog der DNB (Deutsche Nationalbibliothek)

- Verbundkatalog der Öffentlichen Bibliotheken im GBV (Gemeinsamer Bibliotheksverbund)

- Gesamtkatalog des GBV (Gemeinsamer Bibliotheksverbund)

Die einzelnen Arbeitsschritte zur Recherche in den Datenbanken und zur Datenübernahme werden in den folgenden Kapiteln erläutert.

Das Thema "Installation“ ist nicht Bestandteil dieser Anleitung, sondern wird der Installationsanleitung des Kernsystems behandelt.

\section{Programmstart}

Zum Starten des Programms wählen Sie im Startmenü unter allegro-OEB

2/Fremddatenübernahme die Fremddatenübernahme für die gewünschte Datenbank aus:

- $\quad$ Fremddatenübernahme - Erwerbung

- Fremddatenübernahme - Katalog

\subsection{Die Anmeldung}

Wie alle allegro-OEB-Programme ist auch die allegro-OEB Fremddatenübernahme mit einem Anmeldedialog versehen.

Es handelt sich bei der Anmeldung um eine einmalige Tagesanmeldung. Bei der ersten Funktion, die Sie aufrufen, erscheint der Anmeldedialog. Wenn Sie sich hier erfolgreich angemeldet haben, gilt die Anmeldung für den gesamten Tag und für alle weiteren allegro-OEB-Programme. Wenn Ihre Tagesanmeldung noch gültig ist, erscheint diese Seite nicht und Sie werden sofort zum Suchformular weitergeleitet.

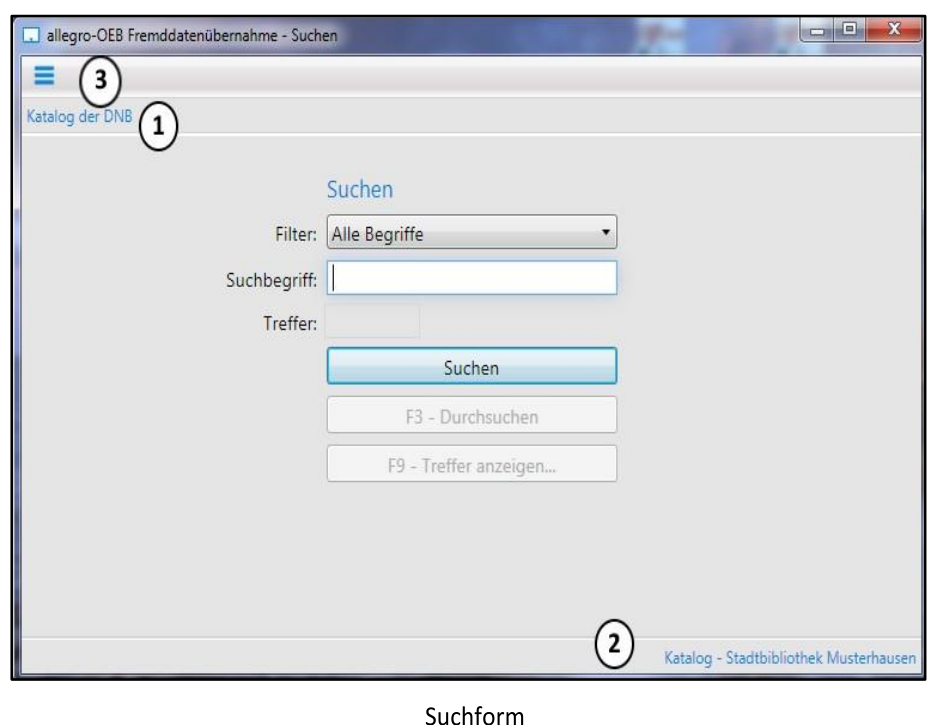

Im oberen Bereich (1) finden Sie den Namen der Fremddatenbank, im unteren Bereich (2) die lokal ausgewählte Datenbank - Katalog oder Erwerbung - und den Namen der Zweigstelle, unter der Sie angemeldet sind. Klicken Sie auf das Symbol links oben (3), öffnet sich das Menü. 


\subsection{Einstellungen}

Bei der ersten Nutzung des Programms sind zunächst die Einstellungen zu überprüfen und entsprechend anzupassen. Wählen Sie den Punkt „Einstellungen“ im Menü aus oder drücken Sie F2.

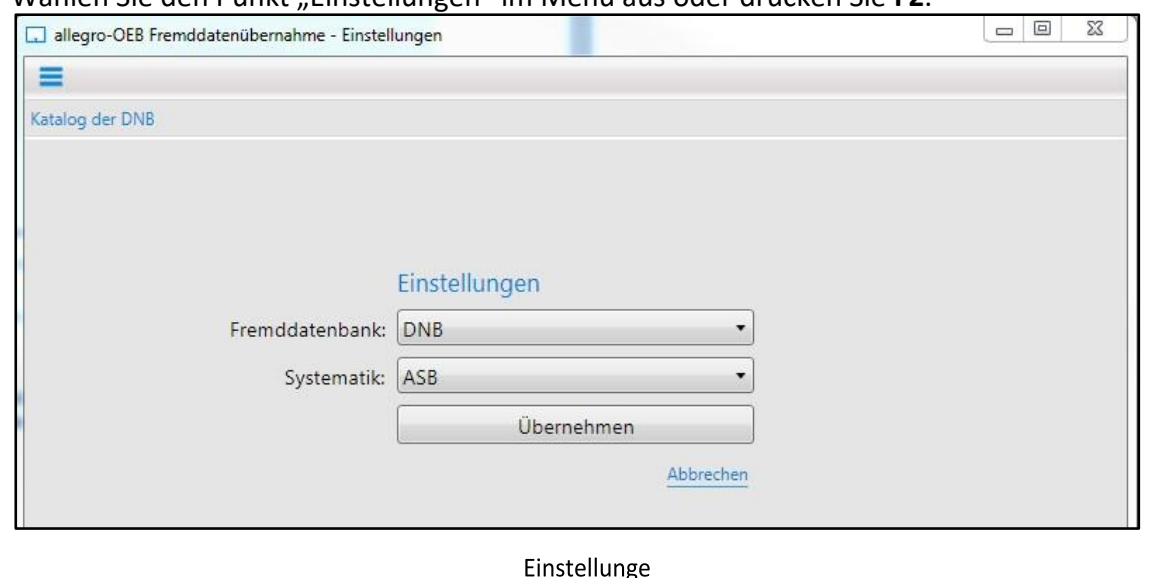

Wählen Sie die gewünschte Fremddatenquelle aus und stellen Sie die gewünschte Systematik ${ }^{198}$ ein, die bei der Datenübernahme berücksichtigt werden soll.

Folgende Fremddatenbanken stehen zur Auswahl:

- OEVK im GBV (Verbundkatalog der Öffentlichen Bibliotheken im GBV)

- DNB (Katalog der Deutschen Nationalbibliothek)

- GVK im GBV (Gesamtverbundkatalog des GBV)

Mit „Übernehmen“ werden die Einstellungen übernommen und bleiben zukünftig als Grundkonfiguration erhalten. Das Ändern der Einstellungen erfolgt immer über das Menü „Einstellungen“ bzw. F2. In den meisten Fällen ist das Ändern der Einstellungen aber nicht erforderlich.

\subsection{Schriftgröße anpassen}

Über die Menüeinträge „Schriftgröße +" und „Schriftgröße -“ bzw. über die Tastenkombination STRG+ / STRGkönnen Sie eine andere Schriftgröße einstellen. Diese gilt für alle Bereiche der allegro-OEB Fremddatenübernahme und bleibt auch nach einem Programmneustart erhalten.

\section{Recherche und Trefferliste}

Im Feld „Suchbegriff“ geben Sie den gewünschten Begriff ein. Dies kann ein Verfasser, Titel, Reihentitel, Schlagwort oder die ISBN sein. Klicken Sie auf "Suchen“ oder starten Sie die Anfrage mit ENTER.

Die Anzahl der Treffer wird angezeigt. Über den Button „F9 - Treffer anzeigen...“ oder direkt mit F9 gelangen Sie zur Trefferliste. Hier werden max. 100 Treffer angezeigt.

\footnotetext{
${ }^{198}$ Nicht alle angebotenen Titeldaten enthalten eine systematische Auswertung.
} 


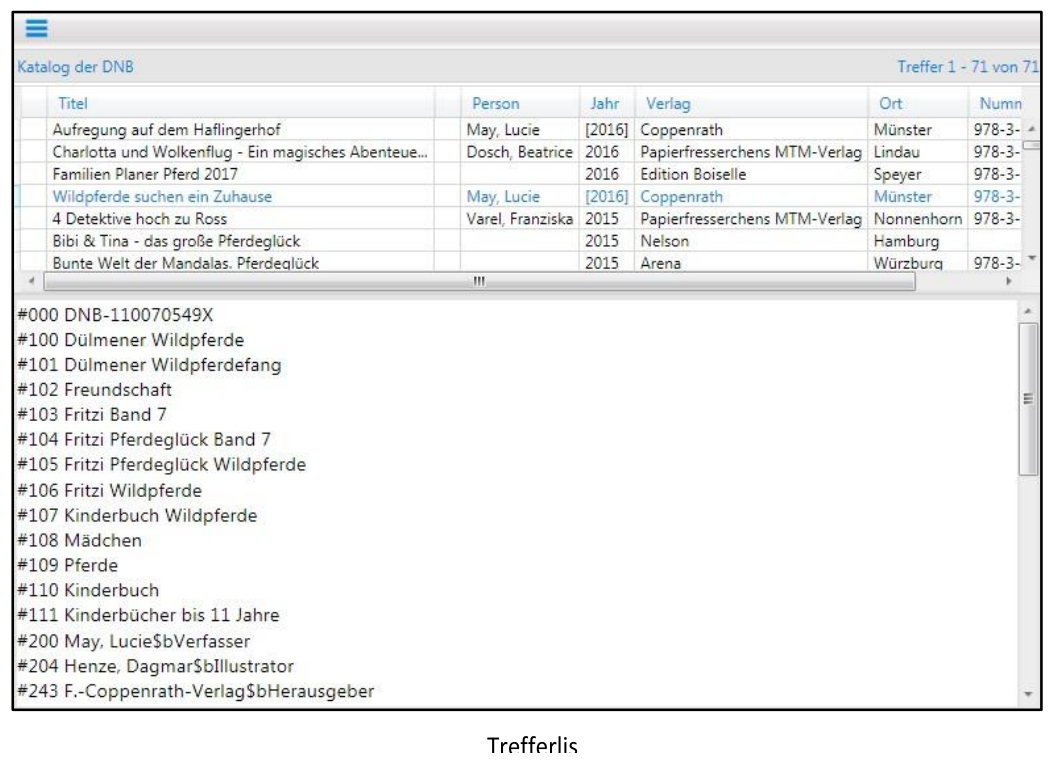

\subsection{Treffermenge reduzieren}

Ist die Treffermenge zu groß, kann diese über das Menü in der Trefferanzeige mit „Suche verfeinern“ oder F3 eingegrenzt werden. Sie gelangen dann wieder zum Suchfenster. Tippen Sie hier - unter Beibehaltung der bestehenden Treffermenge - einen neuen Suchbegriff ein und klicken Sie dann auf „F3 - Durchsuchen“ oder drücken Sie die Taste F3. Die zuvor ermittelte Treffermenge wird nun durchsucht.

\subsection{Suchfilter einstellen}

Es besteht außerdem die Möglichkeit, die Suche jederzeit auf bestimmte Felder - Verfasser, Titel, Verlag, etc. einzugrenzen.

Wählen Sie dafür einen entsprechenden Filter aus.

\begin{tabular}{|l|}
\hline Alle Begriffe \\
\hline Alle Begriffe \\
\hline Person, Verfasser \\
Titel \\
Nummer (ISBN, ISSN) \\
Ort, Verlag \\
Erscheinungsjahr \\
Schlagwort \\
\hline
\end{tabular}

Filtereinstellungen

Mit der Auswahl eines Filters schränken Sie den Suchbereich von Anfang an ein oder verfeinern die Suche, um somit die Anzahl der Treffer zu verringern.

\subsection{Recherche über ISBN}

Die einfachste und genaueste Suchmethode ist die Recherche über die ISBN. Die Nummer kann mit oder ohne Bindestriche eingegeben werden. Je nach Scannerkonfiguration können Sie die ISBN auch mit dem Handscanner einlesen. Das Einstellen eines Filters ist hierbei nicht zwingend erforderlich.

Wenn Ihre Recherche genau einen Treffer ergibt, gelangen Sie über den Button „F9 - Treffer anzeigen...“ oder direkt mit $\mathbf{F 9}$ in das Bearbeitungsfenster ${ }^{199}$ und können den Datensatz direkt überarbeiten und/oder importieren.

\subsection{Neue Suche}

Um eine neue Suche zu starten, kehren Sie mit F4 oder über das Menü mit „Neue Suche“ von der Trefferliste zum Suchfenster zurück.

${ }^{199}$ s. Kapitel 4.1. 


\section{Auswahl und Import der Daten}

Wählen Sie in der Trefferliste den gewünschten Titel aus. Entweder klicken Sie diesen mit einem Doppelklick direkt an oder Sie blättern mit den Pfeiltasten zum gewünschten Eintrag und wählen diesen mit ENTER aus.

Der Datensatz wird nun in einer Zwischenablage im allegro-OEB-Kategorienformat angezeigt und kann an dieser Stelle bearbeitet werden. Zum Nachbearbeiten der Titel finden Sie ausführliche Hinweise im nächsten Kapitel.

Zum Übernehmen des Datensatzes drücken Sie F5 oder klicken Sie auf „F5 - Importieren“. Sofern keine Fehler gemeldet werden, kehrt das Programm nach dem Import sofort zur Trefferliste zurück.

\subsection{Dublettencheck}

Beim Importieren der Daten erfolgt eine Dublettenprüfung über die Titelidentnummer. Existiert ein Datensatz mit der Titelidentnummer bereits in der lokalen Datenbank, erscheint folgender Hinweis:

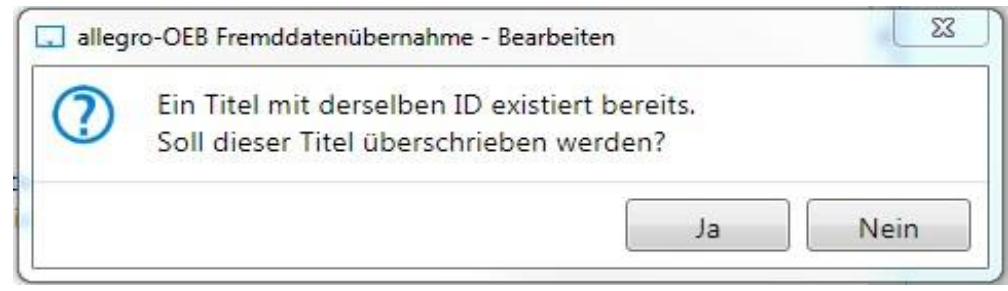

Dublettencheck

„Nein“ ist hier als Option vorgegeben. Mit ENTER bestätigen Sie, dass der Titel nicht überschrieben wird. Überprüfen Sie zunächst direkt den Titel im Katalog bzw. der Erwerbung.

Wenn Sie den vorhandenen lokalen Titel durch den neuen Datensatz ersetzen möchten, wählen Sie „Ja“.

\section{Datenbearbeitung}

Zum Bearbeiten der Titeldaten haben Sie zwei Möglichkeiten:

1. die direkte Bearbeitung im Programm „Fremddatenübernahmen“ vor dem Import der Daten

2. die Bearbeitung im Editor der allegro-OEB Katalogisierung nach dem Import der Titeldaten, der Zeitpunkt ist dabei irrelevant. Die Bearbeitung kann direkt im Anschluss erfolgen oder gebündelt, wenn alle Titel importiert wurden.

Welche Variante Sie wählen, hängt davon ab, wie gut die Datenqualität ist und welche hausinternen Ergänzungen ggf. noch notwendig sind.

Die Daten der DNB sind in der Regel qualitativ sehr hochwertig und es muss im Normalfall nur die Signatur ergänzt werden. Dies kann beispielsweise vor der Fremddatenübernahme erfolgen.

Müssen darüber hinaus noch weitere Felder ergänzt werden, z. B. der Interessenkreis oder weitere Schlagworte, kann es sinnvoll sein, die Bearbeitung nach dem Import in der allegro-OEB Katalogisierung zu erledigen.

Inventarisierung: Unabhängig davon, für welche Vorgehensweise Sie sich entscheiden, muss auf jeden Fall nach der Datenübernahme der Titel die Inventarisierung erfolgen.

Diese führen Sie wie gewohnt in der Katalogisierung durch. Das kann direkt im Anschluss erfolgen - was sinnvoll ist, wenn die Nachbearbeitung in der Katalogisierung erfolgt - oder gebündelt nach Abschluss aller Titelimporte - wenn die Bearbeitung z. B. direkt im Programm „Fremddatenübernahme“ erledigt wird und der ständige Programmwechsel zu unkomfortabel ist.

\subsection{Bearbeitung im Programm „Fremddatenübernahme“}

Wenn Sie den gewünschten Titel aus der Trefferliste ausgewählt haben, wird dieser im allegro-OEBKategorienformat angezeigt. 


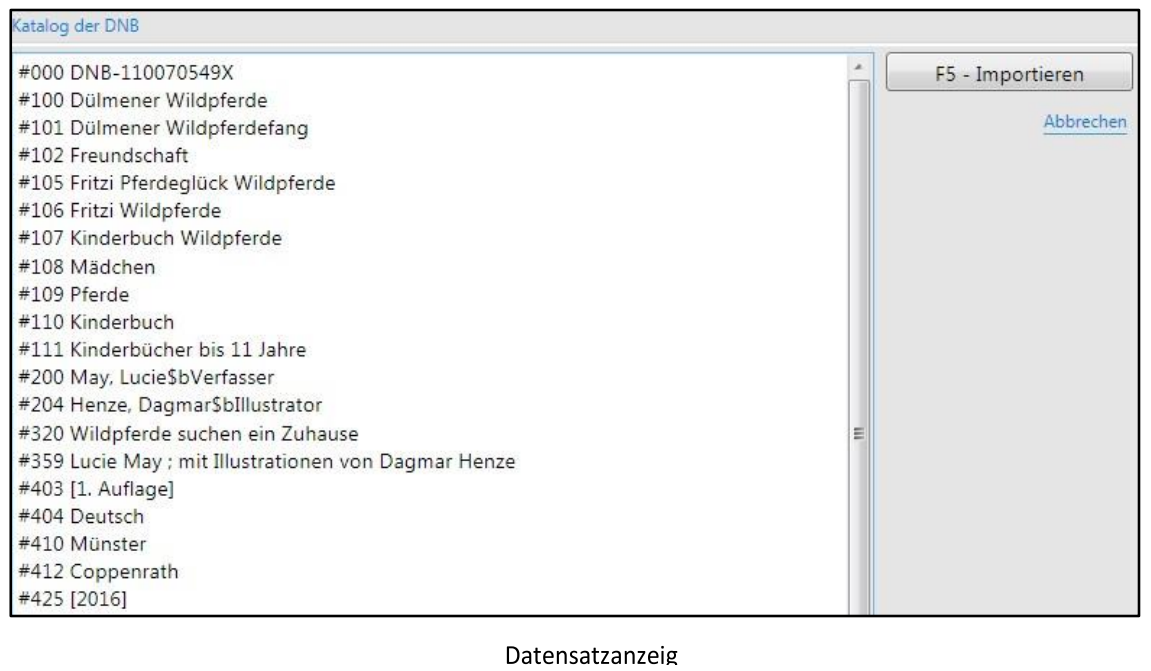

Hier können Sie Kategorien löschen, korrigieren und hinzufügen. An dieser Stelle kann nur mit Kategorienummern gearbeitet werden, d. h. Sie müssen die entsprechenden Nummer wissen. Ansonsten entspricht die Funktionsweise der Datenbearbeitung der eines Texteditors.

Wenn Sie beispielsweise die Signatur ergänzen möchten, gehen Sie an das Ende des Dokuments und ergänzen in einer neuen Zeile die \#060 mit entsprechendem Inhalt.

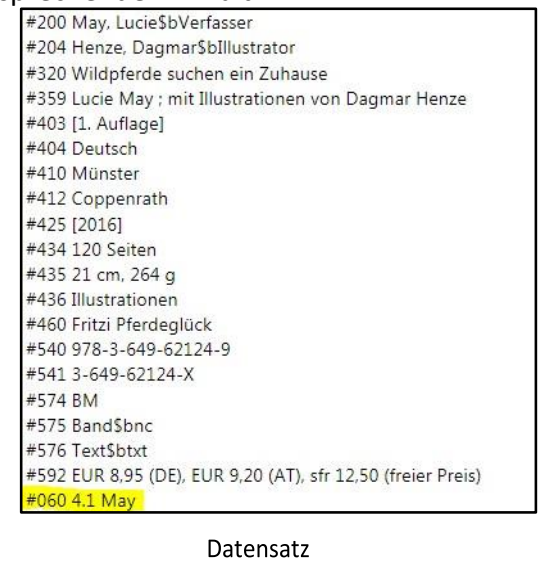

Wenn Sie bei der Nachbearbeitung an dieser Stelle weitere Personen mit Beziehungskennzeichnungen ergänzen möchten, geben Sie vor der Beziehungskennzeichnung \$b ein:

\#200 May, Lucie\$bVerfasser

\#204 Henze, Dagmar\$blllustrator

Ist die Bearbeitung abgeschlossen, können Sie den Datensatz importieren und anschließend das Exemplar im Katalog inventarisieren.

\subsection{Bearbeitung im Programm „Katalogisierung“}

Bei der Nachbearbeitung von mehreren Elementen einer Titelaufnahmebietet sich die Bearbeitung in der allegro-OEB Katalogisierung an.

Recherchieren Sie zunächst den importierten Titel - beispielsweise über die ISBN - und bearbeiten Sie den Datensatz wie gewohnt mit F3.

Sie können den Datensatz nun im Editor bearbeiten, indem Sie einzelne Kategorien hinzufügen. Alternativ können Sie im Editor erneut die Vorlage zur Erfassung von z. B. „Buch Monografie“ auswählen und arbeiten die Änderungen anhand der Maske ein. Hierbei erkennen Sie leicht, welche Kategorien noch fehlen.

Ist die Bearbeitung abgeschlossen, können Sie den Datensatz speichern. Anschließend muss noch die Inventarisierung des Exemplars erfolgen. 
Anhang 8: allegro-OEB.Dokumentation.Fremddatenübernahme

5. Konfiguration

\subsection{Proxy-Server}

Um die allegro-OEB Fremddatenübernahme mit einem Proxyserver mit Authentifizierung zu nutzen, müssen die Proxy- und Authentifizierungsdaten in der Datei proxy.config hinterlegt werden. Die Datei befindet sich nach dem ersten Start des Programms in Dokumente/allegro-OEB/SRU/Config und hat folgendes Format:

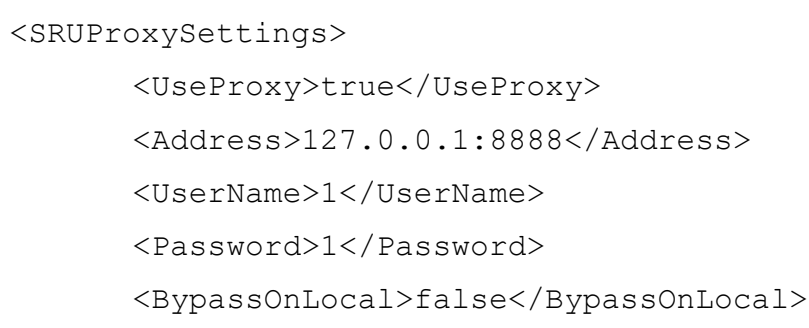

Darüber hinaus besteht die Möglichkeit, die Datei proxy .config in einem zentralen Verzeichnis abzuspeichern, beispielsweise im Verzeichnis C: \ALLEGRO\SRU.

Dieses Verzeichnis kann mit folgender Option aufgerufen werden:

/configfolder="C: \ALLEGRO\SRU"

Bitte beachten Sie die Groß- und Kleinschreibung. Leerzeichen im Pfad sind durch Anführungszeichen zu ersetzen. Das „Ziel“ in einer entsprechenden Programmverknüpfung lautet wie folgt:

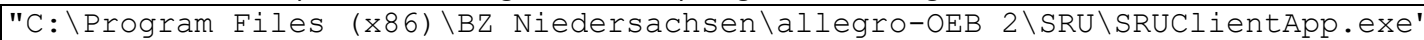
/ database=Katalog / configfolder="C: \ALLEGRO \SRU" 
Verzeichnis der auf der beiliegenden CD gespeicherten Dateien

Verzeichnis der auf der beiliegenden CD gespeicherten Dateien

Gesamttabelle Export easyfeedback.xlsx

DBS gesamt_DBS-Personal.xlsx

Übersicht Fragen_Bibliotheken.xlsx

Bibliotheksmanagementsysteme_Hardware.xlsx

Katalogisierung_Fremddaten.xlsx

Zuordnung Abbildungen

Kiefer-Masterarbeit.pdf 
Selbstständigkeitserklärung

Selbstständigkeitserklärung

Ich erkläre hiermit, dass ich die vorliegende Abschlussarbeit selbstständig verfasst

und die verwendeten Quellen deutlich gekennzeichnet habe.

Ich stimme einer Veröffentlichung durch das WIT zu.

Wildau, 20. März 2019

Andrea Kiefer 